\title{
QED interference in charge asymmetry near the $Z$ resonance at future electron-positron colliders
}

\author{
Stanislaw Jadach ${ }^{*}$ and Scott A. Yost ${ }^{\dagger}$ \\ Institute of Nuclear Physics, Polish Academy of Sciences, ul. Radzikowskiego 152, 31-342 Kraków, Poland
}

(Received 3 December 2018; published 18 July 2019)

\begin{abstract}
The measurement of the charge asymmetry $A_{\mathrm{FB}}\left(e^{-} e^{+} \rightarrow \mu^{-} \mu^{+}\right)$will play an important role at the high-luminosity circular electron-positron collider FCCee considered for construction at CERN. In particular, near the $Z$ resonance, $\sqrt{s} \simeq M_{Z} \pm 3.5 \mathrm{GeV}, A_{\mathrm{FB}}$ will provide a very precise value of the pure electromagnetic coupling constant $\alpha_{\mathrm{QED}}\left(M_{Z}\right)$, which is vitally important for overall tests of the Standard Model. For this purpose, $A_{\mathrm{FB}}$ will be measured at the FCCee with an experimental error better than $\delta A_{\mathrm{FB}} \simeq 3 \times 10^{-5}$, at least a factor of 100 more precisely than at past LEP experiments! The important question is whether the effect of interference between photon emission in the initial and final state (IFI) can be removed from the $A_{\mathrm{FB}}$ data at the same precision level using perturbative QED calculations. A first quantitative study of this problem is presented here, with the help of the KKMC program and a newly developed calculation based on soft photon resummation, matched with NLO and NNLO fixed-order calculations. It is concluded that a factor of 10 improvement with respect to the LEP era is obtained. We also present a clear indication that reducing the uncertainty of charge asymmetry near the $Z$ peak due to IFI down to $\delta A_{\mathrm{FB}} \simeq 3 \cdot 10^{-5}$, i.e. the expected experimental precision at FCCee, is feasible.
\end{abstract}

DOI: $10.1103 /$ PhysRevD.100.013002

\section{INTRODUCTION}

At the future high-energy high-luminosity circular electron-positron collider FCCee [1-3] proposed for construction at CERN, the measurement of the muon charge asymmetry $A_{\mathrm{FB}}^{\mu}=A_{\mathrm{FB}}\left(e^{-} e^{+} \rightarrow \mu^{-} \mu^{+}\right)$will play an additional important role. Near the $Z$ resonance, $\sqrt{s_{ \pm}} \simeq M_{Z} \pm 3.5 \mathrm{GeV}$, the measurement of $A_{\mathrm{FB}}^{\mu}$ may provide a very precise value of the pure electromagnetic coupling constant $\alpha_{\mathrm{QED}}\left(M_{Z}\right)$, which is vitally important for overall tests of the Standard Model (SM), especially for the SM prediction of $\sin ^{2} \theta_{\text {eff }}^{l}$ and $M_{W}$ [4], at a precision level at least a factor of 10 better than presently. This kind of the measurement of $\alpha_{\mathrm{QED}}\left(M_{Z}\right)$ was proposed and analyzed in Ref. [5].

QED interference between photons emitted from the initial and final charged leptons (IFI) had to be taken into account in past measurements of the charge asymmetry $A_{\mathrm{FB}}^{\mu}$ (forward-backward angular asymmetry) at the

\footnotetext{
*stanislaw.jadach@ifj.edu.pl

†Present address: The Citadel, 171 Moultrie St., Charleston, South Carolina 29409, USA.

scott.yost@citadel.edu

Published by the American Physical Society under the terms of the Creative Commons Attribution 4.0 International license. Further distribution of this work must maintain attribution to the author(s) and the published article's title, journal citation, and DOI. Funded by SCOAP.
}

electron-positron colliders LEP and SLC. Let us abbreviate $A_{\mathrm{FB}}^{\mu} \equiv A_{\mathrm{FB}}$. In overall tests of the SM, the measurement of $A_{\mathrm{FB}}$ contributed mainly to knowledge of the $Z$ couplings and/or the effective electroweak mixing angle $\sin ^{2} \theta_{\text {eff }}^{l}$ [6].

Thanks to the very high luminosity of the FCCee [1], the charge asymmetry $A_{\mathrm{FB}}\left(M_{Z} \pm 3.5 \mathrm{GeV}\right)$ will possibly be measured with an error $\delta A_{\mathrm{FB}} / A_{\mathrm{FB}} \simeq 3 \times 10^{-5}$ or even better $[2,5]$. This immediately poses the question of whether the effect of QED initial-final interference can be removed from the data at the same precision level. How big is the effect of IFI in $A_{\mathrm{FB}}$ ? Far from the resonance, it is about 2$3 \%$ and it is even bigger for a tight cutoff on the total energy of the emitted photons. At the top of the $Z$ resonance, where $A_{\mathrm{FB}}$ was measured most precisely in the past LEP experiments, the IFI effect is suppressed by the ratio $\Gamma_{Z} / M_{Z}$ to the level of $\delta A_{\mathrm{FB}} \sim 0.1 \%$, due to the long time separation between the creation and the decay stages of the $Z$ resonance, as elaborated in many LEP era works, see Refs. [7-12]. As we shall see in our analysis, at $\sqrt{s} \simeq M_{Z} \pm 3.5 \mathrm{GeV}$, the same $\Gamma_{Z} / M_{Z}$ suppression of IFI in $A_{\mathrm{FB}}$ still works to some extent, but the IFI effect is nevertheless at the $\delta A_{\mathrm{FB}} \sim 1 \%$ level, and growing for tight cutoffs, in spite of partial cancellations in the difference between values at $\sqrt{s} \simeq M_{Z} \pm 3.5$, as already noticed in Ref [5].

This effect is huge with respect to the planned experimental precision at FCCee, and it would render measurement of the $A_{\mathrm{FB}}\left(M_{Z} \pm 3.5 \mathrm{GeV}\right)$ completely useless unless 
the theoretical evaluation of IFI is equally precise! Note that in the LEP data analysis, a cutoff on the total photon energy was imposed by requiring a minimum value of the effective mass of the muon pair, $M_{\mu^{-} \mu^{+}}$, or a maximum acollinearity angle, which was translated into an upper limit on the total photon energy $E_{\gamma}^{\text {tot }} / s^{1 / 2}$ varying between 0.5 to 0.998 (see Table 2.1 in Ref. [6]). Due to the higher precision of the FCCee, a stronger cutoff probably will be preferred in order to minimize the background from hadronic and tau pair channels, and for better control of the angular dependence of the muon detector efficiency. ${ }^{1}$ Also, theoretical control over IFI may be better for a stronger cutoff (in spite of its larger size) thanks to the power of soft photon resummation and a better elimination of the four fermion contributions. For this reason, we will use a photon energy cutoff $E_{\gamma}^{\text {tot }} / s^{1 / 2} \leq 0.2$ or stronger.

How precise are the theoretical evaluations of the effect of IFI in $A_{\mathrm{FB}}$ presently available in perturbative QED? In the pre-LEP era, $\mathcal{O}\left(\alpha^{1}\right)$ fixed-order calculations were quoted to provide $\sim 0.3-0.5 \%$ precision; see the review of Ref. [7]. In the LEP1 phase near the $\mathrm{Z}$ resonance, thanks to $\Gamma_{Z} / M_{Z}$ suppression, the IFI effect in $A_{\mathrm{FB}}$ at the $Z$ peak was not a burning issue. For instance, in the work of Ref. [10] used in the final data analysis of LEP1 of Ref. [6], the calculations of the IFI effect were done using ZFITTER [13] and TOPAZ0 $[14,15]$ programs, cross checking them with the KORALZ Monte Carlo [16,17].

In all these calculations and programs, the treatment of IFI was at the $\mathcal{O}\left(\alpha^{1}\right)$ fixed-order level, without soft photon resummation. Pioneering work on the resummation of soft photon effects near a narrow resonance, including resummation of $\ln \left(\Gamma_{Z} / M_{Z}\right)$, was already done earlier by the Frascati group, see Refs. [18-20], but it was not exploited in the above studies, mainly because they did not include hard photon effects in a realistic way.

Significant progress on the IFI issue was made just before the end of the LEP era, with the advent of new method of the soft photon resummation matched with fixed-order QED corrections up to $\mathcal{O}\left(\alpha^{2}\right)$ and electroweak (EW) corrections up to $\mathcal{O}\left(\alpha^{1}\right)$, the so-called coherent exclusive exponentiation [12,21] (CEEX) and its implementation in the KKMC program [22]. The CEEX implementation in KKMC has included all the advances of soft photon resummation of the IFI contributions of Refs. [18-20] relevant for narrow resonances. ${ }^{2}$ The SM predictions of $\mathrm{KKMC}$ for $A_{\mathrm{FB}}$ and other experimental observables were possible for arbitrary event selections (cuts), because KKMC is a regular Monte Carlo (MC) event generator. Correct matching of the $\mathcal{O}\left(\alpha^{1}\right)$ IFI contributions with other non-IFI corrections up to complete $\mathcal{O}\left(\alpha^{2}\right)$ QED and $\mathcal{O}\left(\alpha^{1}\right)$ electroweak was implemented throughout the

\footnotetext{
${ }^{1} \mathrm{P}$. Janot, private communication.

${ }^{2}$ In particular, resummation of $\ln \left(\Gamma_{Z} / M_{Z}\right)$ was included.
}

entire multiphoton phase space, including any number of soft and hard photons.

The CEEX/KKMC calculation was instrumental in the analysis of LEP2 data above the $Z$ peak and near the $W W$ threshold, and helped to consolidate data analysis of $e^{-} e^{+} \rightarrow f \bar{f}$ processes near the $Z$ peak. The precision of the IFI calculations quoted at the end of the LEP era was $\delta A_{\mathrm{FB}} \simeq 0.1 \%$ at the $Z$ peak and $\delta A_{\mathrm{FB}} \simeq 0.3 \%$ far away from the $Z$ resonance; see Refs. [10-13,23]. These papers represent the state of the art in the perturbative QED calculation of the IFI contributions to $A_{\mathrm{FB}}$ until the present day.

The KKMC precision tag on the IFI calculations, both near the $Z$ peak and away from it, was more than sufficient for analyzing all LEP experimental data at the end of the LEP era. However, this precision was quite clearly underestimated, i.e. most likely it was far better. However, it was difficult to better quantify the theoretical uncertainty of the IFI prediction of KKMC, because there was no other calculation at a similar level of sophistication to compare with. One of the main aims of this work will be to develop a new alternative numerical calculation of the IFI contribution, in order to compare with KKMC and quantify theoretical uncertainties of the IFI component in $A_{\mathrm{FB}}$ at the precision level higher than presently available.

Generally, one may be quite skeptical whether an improvement of the QED calculation of IFI in $A_{\mathrm{FB}}$ from the LEP-era $\delta A_{\mathrm{FB}} \sim 10^{-3}$ down to $\delta A_{\mathrm{FB}} \sim 10^{-5}$, i.e. by a factor of 100 , is feasible at all! However, there is an interesting precedence-the prediction of perturbative QED for the $Z$ line shape also progressed by a similar factor from the time before LEP started until the end of the LEP era. This was possible mainly due to soft photon resummation techniques. The use of these techniques is again critical for the present task of improving the QED calculation of IFI in $A_{\mathrm{FB}}$. The aim of this paper is to check how far we can advance on the road to the precision required for FCCee.

Let us stress that present work is not a progress report on the development of KKMC, simply because KKMC remains the same as in 1999. It is, however, definitely a progress report on the understanding of the IFI contribution, thanks to several newly developed analytic calculations implemented in the new computer code KKFoam and a wealth of numerical results for various kinds of matrix elements, phase space integrations, cutoffs, etc. This work will have to be continued in the future, including possible upgrades of the matrix element in $\mathrm{KKMC}$, and/or development of new MC programs even more sophisticated than KKMC.

Finally, in view of the growing interest in the higher order SM calculations which would match the very high precision of the FCCee experiment [4,24], it is important to note that the CEEX methodology of photon resummation and matching with fixed-order nonsoft QED and EW corrections also addresses some important issues in the 
QED + EW perturbative calculations, beyond what was typically done for the LEP data analysis, as quoted above. The basic issue is that of the separation of QED and pure EW parts of the SM in the perturbative expansion. This is necessary, because QED corrections are larger, and their soft part has to be resummed to infinite order while the nonsoft part must be included up to $\mathcal{O}\left(\alpha^{4}\right)$, while the perturbative series for more complicated EW corrections can be truncated earlier, at $\mathcal{O}\left(\alpha^{2}\right)$ or $\mathcal{O}\left(\alpha^{3}\right)$ [24].

In the calculations for LEP data analysis (see [10] and other Refs. quoted above), the issue of separating QED and EW parts was not a critical one, because resummed higherorder QED was typically confined to the initial state radiation (ISR) effective radiator function, and in the remaining $\mathcal{O}\left(\alpha^{1}\right)$ calculations, the QED and EW parts enter additively, and thus are well separated [except of negligible IFI which was controlled up to $\left.\mathcal{O}\left(\alpha^{1}\right)\right]$. Beyond $\mathcal{O}\left(\alpha^{1}\right)$, the QED and EW parts often enter multiplicatively, for instance in 2-loop graphs with one loop involving photon exchange and another loop with massive bosons or fermions, or one loop of pure EW origin with a hard photon emission insertion. The CEEX technique provides for clear methodology of separating/factorizing QED and EW parts at any order.

Omitting algebra which can be found in Refs. [12,21], the main points of CEEX methodology can be summarized as follows:

(i) In the first step of the factorization of the infrared (IR) factors at the amplitude level, for any group of multiloop graphs with one photon insertion, the IR part is subtracted at the amplitude level using a wellknown (1-loop) function defined in the classic Yennie-Frautschi-Suura work [25] times a finite contribution one order lower without a photon insertion. (A similar procedure applies for multiloop corrections with two and more photon insertions.) The remaining finite non-IR remnant will be used in the next step. Similarly, for any group of real photon insertions into a given multiloop diagram with pure EW content, one subtracts, at the amplitude level, a well-known eikonal factor times a basic diagram with pure EW content.

A similar well-defined procedure applies for amplitudes with more real and virtual photon insertions. The first step is finalized by constructing spin amplitudes for an arbitrary number of real photons distributed over the entire phase space in which non-IR remnants after IR subtractions are reinserted in a well-defined way, while IR virtual factors are exponentiated and explicit IR-divergent eikonal factors are ready for MC integration in the next step. ${ }^{3}$

${ }^{3}$ Collinear contributions giving rise to nonsoft mass logarithms are included order-by-order in the present version of CEEX. (ii) The second step of resummation, that of squaring spin amplitudes, spin summation and phase space integration, is done numerically in the Monte Carlo event generator. (There is no possibility of doing it analytically.) In the above CEEX scheme, the bulk of the IFI contribution is present in the resummed/ exponentiated real + virtual form-factor and in the interferences emerging from squaring multiphoton spin amplitudes. Smaller contributions will remain hidden in the nonsoft finite remnants. ${ }^{4}$ The treatment of the nonfactorizable $\gamma \gamma$ and $\gamma Z \mathcal{O}\left(\alpha^{1}\right)$ boxes in the above resummation scheme can be seen explicitly in Eqs. (29), (33) for the matrix element in KKFoam and in Eqs. (21-24) of Ref. [22] for the CEEX matrix element in KKMC.

A somewhat more detailed overview of the CEEX factorization and resummation in QED is given in Secs. C.2.7 and C.3 of the recent review [24], while complete details can be found in Refs. [12,21,22]. The above CEEX factorization and resummation of the universal QED corrections will work equally well for extensions of the SM (BSM), provided the BSM predictions are formulated at the amplitude level. So far, KKMC for the $e^{-} e^{+} \rightarrow f \bar{f}+n \gamma$ process is the only implementation of the CEEX scheme. In Ref. [26], it is argued that in the context of the FCCee project, it is urgent to implement it also for the Bhabha process.

The so-called deconvolution of QED effects from LEP experimental data which was instrumental in the construction of pseudo-observables $[6,10]$ can also be reorganized using the CEEX technique. In the LEP version, it was done using ZFITTER, TOPAZ0 and KORALZ, and it was proven to be acceptable within the LEP precision goals [10], but the validity of this procedure is not automatically granted for FCCee precision. In Sec. C.3 in Ref. [24], a proposal is made for extending it to higher precision by exploiting the CEEX factorization and resummation scheme in the MC implementation. An updated discussion on the same theme is also included in Sec. 5.7 of a more recent paper, Ref. [27]. Validation of a more powerful scheme of removing QED effects from experimental data at the precision level of the FCCee experiments will require a lot of numerical studies of the type done in Ref. [10], and most likely the development of the MC programs more powerful and versatile than KKMC.

The plan of the paper is the following: Sec. II explains the origin and character of the IFI effect in the angular distribution of the $e^{-} e^{+} \rightarrow \mu^{-} \mu^{+}$process.

Section III describes a new partly analytic, partly numerical calculation in the semisoft approximation and

\footnotetext{
${ }^{4}$ For instance, spin amplitudes of the $\gamma-Z$ box are split into an IR-divergent part, which is moved to an exponentiated formfactor, and the remaining IR-finite remnants are incorporated in the multiphoton spin amplitudes.
} 

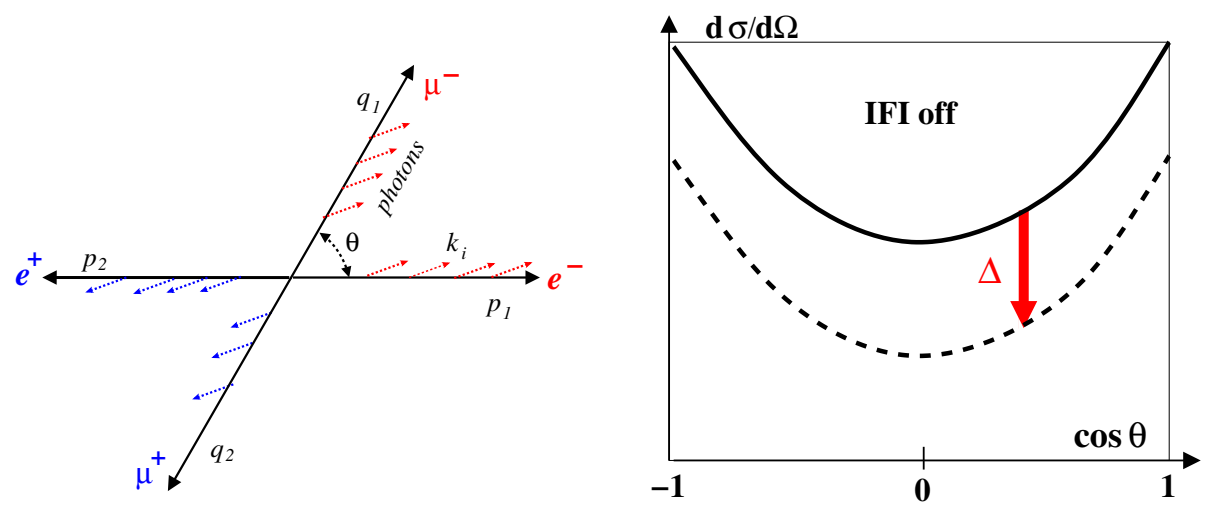

FIG. 1. Multiple photon emission at a wide scattering angle.

its software implementation, KKFoam. "Semisoft" means that the upper limit on the total photon energy is smaller than the total center-of-the mass energy $s^{1 / 2}$, but in the presence of narrow resonance $Z$ it can be smaller or bigger than $\Gamma_{Z} / M_{Z}$.

Multiphoton spin amplitudes (spin amplitudes) are defined (Sec. III A), in such a way that they reproduce the CEEX matrix element in the semisoft regime. Squaring and summing spins is also done analytically, and finally, a phase space integration over photon angles and energies is also performed analytically keeping total real photon energy fixed (Secs. III B and III C). Multiple sums over photons and phase space integrations are done (exactly) in a straightforward way, with a minimal use of Mellin-Fourier transforms. ${ }^{5}$

The IFI effect appears in the resulting muon angular distribution. The final analytic result involves a fourfold convolution over radiator functions of the initial state radiation (ISR), final state radiation (FSR) and two functions due to initial-final state interference (IFI) (Sec. III C). The remaining integration over the phase space is delegated to a numerical Monte Carlo method.

Validity of the formula is formally extended to the full phase space, such that the ISR and FSR radiator functions can be upgraded with the known nonsoft QED contributions up to $\mathcal{O}\left(\alpha^{2}\right)$ (Sec. III D). Electroweak $\mathcal{O}\left(\alpha^{1}\right)$ corrections are also included at the same level as in KKMC, that is using the DIZET library [28]. The remaining 5-dimension integration over the fourfold convolution and muon angle is slightly reorganized for numerical integration (Sec. IIIE) using the universal FOAM MC tool $[29,30]$ for integration and simulation. The resulting MC generator KKFoam is ready for use in Sec. IV.

With all the above distributions and technicalities in place, a wealth of numerical results produced by KKMC and

\footnotetext{
${ }^{5}$ Mellin transforms are used merely as generating functionals for reorganizing combinatorics of the multiple sums over photons.
}

KKFOam is presented in Sec. IV. In particular, in Sec. IV C it will be checked in the calibration exercise that for the matrix elements with resummation and without IFI, the three programs KKMC, KKFOam and KKsem agree within $\sim 10^{-5}$ precision for $\sigma_{\mathrm{tot}}$ and $A_{\mathrm{FB}}$. Note that in the case where IFI is switched off, this kind of comparison of KKMC with the numerical tool KKsem based on analytic exponentiation was already done in Ref. [12]. The new thing here is the inclusion of the IFI.

In Sec. IV D, the IFI effect in $A_{\mathrm{FB}}$ will be examined for three energies $\sqrt{s}=10,87.9,94.3 \mathrm{GeV}$ as a function of the cutoff on total photon energy, comparing results of KKMC and KKFoam. In Sec. IV E, we shall focus on the difference of $A_{\mathrm{FB}}$ between $\sqrt{s_{+}}=94.3 \mathrm{GeV}$ and $\sqrt{s_{-}}=87.9 \mathrm{GeV}$, which is directly related to the measurement of $\alpha_{\mathrm{QED}}\left(M_{Z}\right)$. Sections IV F and IV G will be devoted to estimating higher-order QED uncertainties by means of comparing results for $A_{\mathrm{FB}}$ for several variants of the $\mathrm{QED}$ matrix element in KKMC.

Section V summarizes the results, focusing on the uncertainties in the QED calculation of the IFI effect in $A_{\mathrm{FB}}$.

Three Appendices include details of the analytic phase space integration in the semisoft approximation (Appendix A), the kinematical mapping used in KKFOam for the four-fold convolution (Appendix B) and a recollection of some old $\mathcal{O}\left(\alpha^{1}\right)$ analytic formulas without exponentiation (Appendix $\mathrm{C}$ ) to be used in the numerical studies in Sec. IV.

\section{THE PHYSICS OF IFI}

Any efficient evaluation of IFI in perturbative QED must be based on a good understanding of the basic physics governing this phenomenon. Let us consider the process $e^{-} e^{+} \rightarrow \mu^{-} \mu^{+}$accompanied with any number of real and virtual photons, illustrated schematically in Fig. 1. In the case of final fermions emitted at wide angles, IFI can be neglected, and in the case of total photon energy (ISR + FSR) limited to $K<E=\sqrt{s} / 2$, the angular 

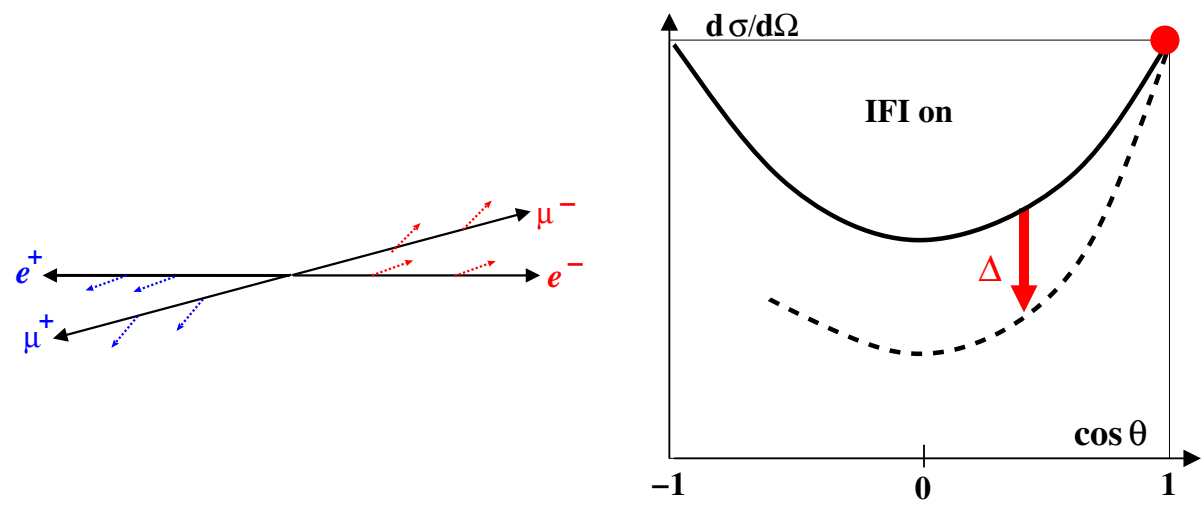

FIG. 2. Multiple photon emission at a forward scattering angle.
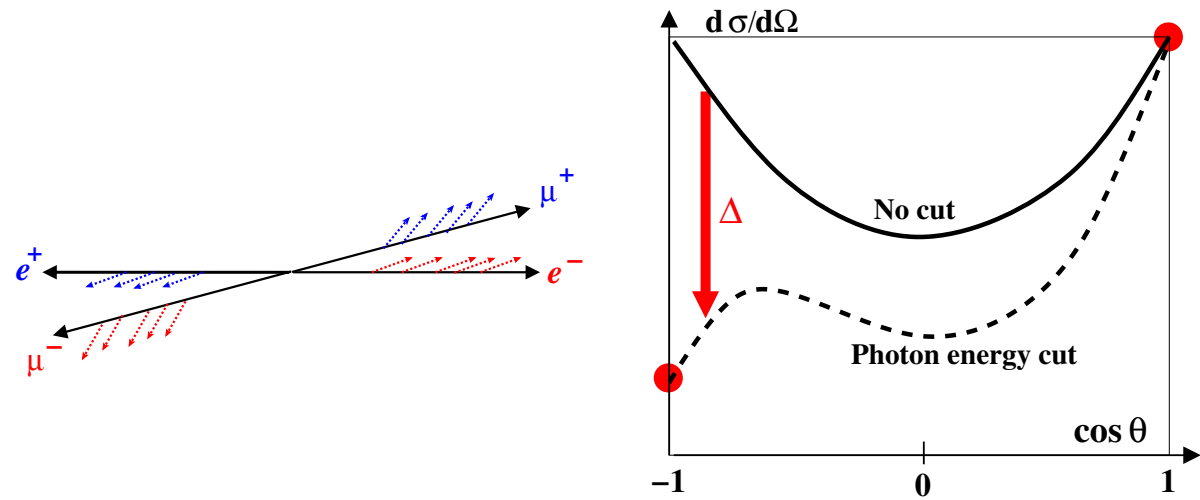

FIG. 3. Multiple photon emission at a backward scattering angle.

distribution is uniformly lowered by a $\theta$-independent Sudakov form factor, ${ }^{6}$

$$
\begin{aligned}
\frac{d \sigma}{d \cos \theta}(K) & \simeq \frac{d \sigma_{\text {Born }}}{d \cos \theta} \exp \left[-\int_{K}^{E} \frac{d k^{0}}{k^{0}}\left(2 \frac{\alpha}{\pi} \ln \frac{s}{m_{e}^{2}}+2 \frac{\alpha}{\pi} \ln \frac{s}{m_{\mu}^{2}}\right)_{\text {virt }}\right] \\
& =\frac{d \sigma_{\text {Born }}}{d \cos \theta} e^{-\Delta(K / E)} .
\end{aligned}
$$

The above relation is illustrated schematically in Fig. 1.

Photon emission is, however, suppressed in the smallangle limit $\theta \rightarrow 0$, as illustrated schematically in Fig. 2, simply because the outgoing muon inherits most of the electromagnetic field accompanying the incoming electron of the same charge as the muon; hence there is no need for the compensating action of bremsstrahlung. In fact, bremsstrahlung dies out completely at $\theta=0$, and it is the IFI contribution which kills both ISR and FSR. The virtual form factor in the angular distribution at $t \rightarrow 0$, $s-|t|-|u|=0,|u| \rightarrow s$ becomes

\footnotetext{
${ }^{6}$ The subscript "virt" appears because virtual corrections feature $-\int_{0}^{E}$, while real emissions add $+\int_{0}^{K}$, so that the uncompensated remnant $-\int_{K}^{E}$ is of virtual origin.
}

$$
\begin{aligned}
\Delta & =\int_{K}^{E} \frac{d k^{0}}{k^{0}}\left(2 \frac{\alpha}{\pi} \ln \frac{s}{m_{e}^{2}}+2 \frac{\alpha}{\pi} \ln \frac{s}{m_{\mu}^{2}}-4 \frac{\alpha}{\pi} \ln \frac{|t|}{|u|}\right) \\
& \rightarrow \int_{K}^{E} \frac{d k^{0}}{k^{0}}\left(2 \frac{\alpha}{\pi} \ln \frac{|t|}{m_{e}^{2}}+2 \frac{\alpha}{\pi} \ln \frac{|t|}{m_{\mu}^{2}}\right) \simeq 0 .
\end{aligned}
$$

On the other hand, in backward scattering, illustrated schematically in Fig. 3, the situation is completely different. The electromagnetic field accompanying $e^{-}$has to be replaced by that of $\mu^{+}$, hence the violent compensating action of the bremsstrahlung is much stronger than for wide angles. Here we have $u \rightarrow 0 \quad(c \rightarrow-1$ side $)$, $s-|t|-|u|=0,|t| \rightarrow s$. Thus, IFI enhances the total QED correction by a factor of 2:

$$
\begin{aligned}
\Delta & =\int_{K}^{E} \frac{d k^{0}}{k^{0}}\left(2 \frac{\alpha}{\pi} \ln \frac{s}{m_{e}^{2}}+2 \frac{\alpha}{\pi} \ln \frac{s}{m_{\mu}^{2}}-4 \frac{\alpha}{\pi} \ln \frac{|t|}{|u|}\right) \\
& \rightarrow \int_{K}^{E} \frac{d k^{0}}{k^{0}}\left(4 \frac{\alpha}{\pi} \ln \frac{s}{m_{e}^{2}}+4 \frac{\alpha}{\pi} \ln \frac{s}{m_{\mu}^{2}}\right),
\end{aligned}
$$

creating a dip in the muon angular distribution for backward scattering (in the presence of a cutoff on the total photon energy, as shown previously). 


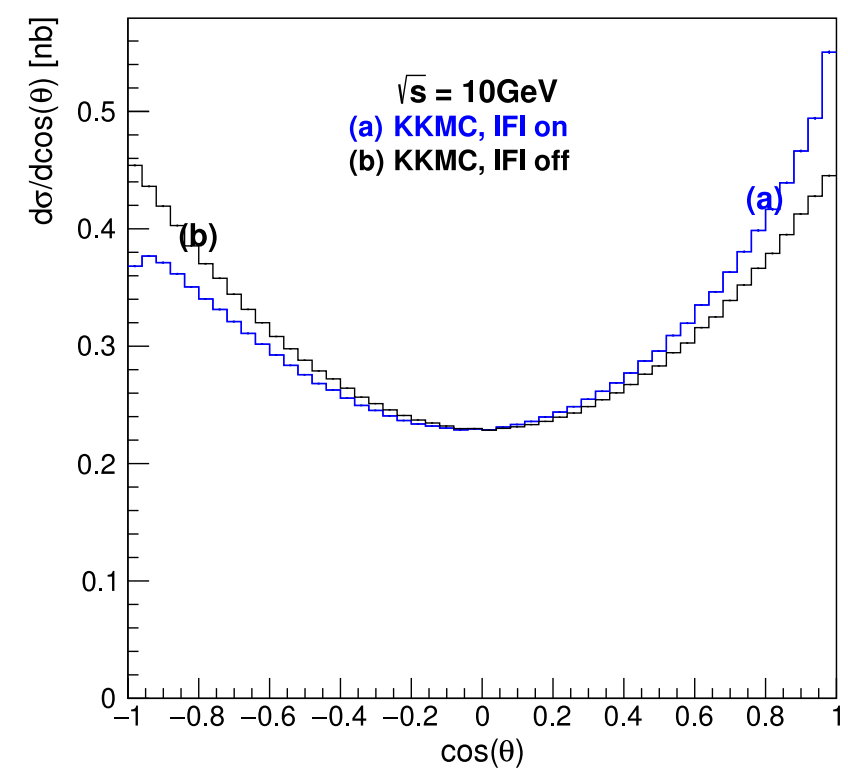

FIG. 4. Muon angular distribution for IFI switched on and off for total photon energy below $2 \%$ of $E_{\text {beam }}=\sqrt{s} / 2=5 \mathrm{GeV}$. The distribution is obtained from KKMC.

In reality, the distribution of $\cos \theta$ far from the resonance appears as shown in Fig. 4 for a relatively strong cutoff on total photon energy ( $2 \%$ of the beam energy).

The presence of a narrow resonance significantly changes the pattern of QED cancellations. Let us analyze briefly how the real and virtual corrections combine at a resonance position $\sqrt{s}=M_{Z}$.

(i) For pure ISR, the virtual correction is $\sim-\frac{2 \alpha}{\pi} \ln \frac{s}{m_{e}^{2}} \ln \frac{E}{\lambda}$, as without a resonance, while the real contribution is cut by the resonance profile $\sim+\frac{2 \alpha}{\pi} \ln \frac{s}{m_{e}^{2}} \ln \frac{\Gamma_{Z}}{\lambda}$. The resulting cross section $\sigma(K)$ is suppressed by the remnant virtual factor $[1-$ $\left.\frac{2 \alpha}{\pi} \ln \frac{M_{Z}}{\Gamma_{Z}}\right]_{\text {virt }}$ for any cut above the resonance width, $K>\Gamma_{Z}$.

(ii) The effect of FSR is the same as in the case without a resonance, i.e. $\sigma(K)$ is suppressed by the remnant virtual factor $\left[1-\frac{2 \alpha}{\pi} \ln \frac{s}{m_{\mu}^{2}} \ln \frac{E}{K}\right]_{\text {virt }}$.

(iii) The case of IFI is most complicated. The virtual correction $\sim-\frac{4 \alpha}{\pi} \ln \frac{t}{u} \ln \frac{\Gamma_{Z}}{\lambda}$ is cut by the resonance (contrary to the ISR case). The real correction $\sim+$ $\frac{4 \alpha}{\pi} \ln \frac{t}{u} \ln \frac{\Gamma_{Z}}{\lambda}$ is also cut by the resonance (similar to the ISR case). The resulting $d \sigma(K) / d \Omega$ is strongly power-suppressed by the $\Gamma_{Z} / M_{Z}$ factor for any cut above the resonance width, $K>\Gamma_{Z}$ ! For an energy cut below the resonance width, $K<\Gamma_{Z}$, IFI starts to rise logarithmically, i.e. the suppression factor is $\sim 1-\frac{2 \alpha}{\pi} \ln \frac{t}{u} \ln \frac{\Gamma_{Z}}{K}$.

Away from the resonance, IFI gradually changes to the previous nonresonant case, and in the entire neighborhood of the resonance, a QED calculation including

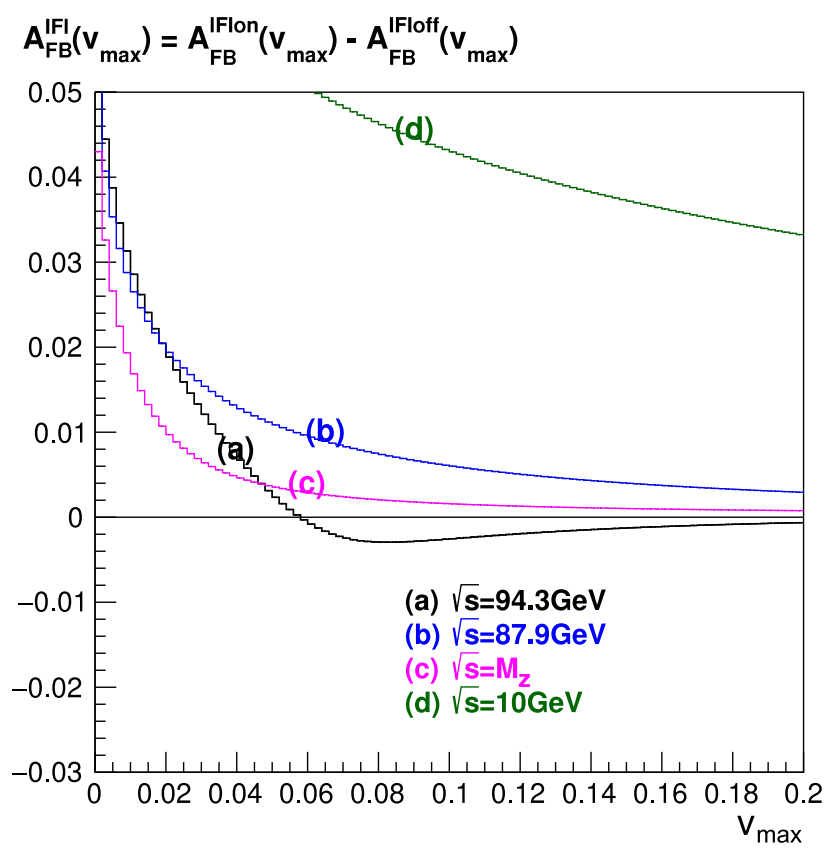

FIG. 5. The dependence of IFI contributions to charge asymmetry on the total photon energy cutoff $v_{\max }$ for various beam energies. The distribution is obtained from KKMC.

photon resummation at the amplitude level (CEEX) is mandatory.

The above mechanism is clearly illustrated in Fig. 5, where the IFI contribution to $A_{\mathrm{FB}}$ is shown as a function of $v_{\max }$, the cutoff on the total photon energy in units of the beam energy. ${ }^{7}$ As we see, far from the $Z$ resonance and for a loose photon energy cutoff, $A_{\mathrm{FB}} \simeq 2 \%$ and grows for stronger cutoffs. In the middle of the resonance, it is strongly suppressed, $A_{\mathrm{FB}}<0.1 \%$, and starts to grow below $v_{\max } \simeq \Gamma_{Z} / M_{Z} \simeq 0.02$. Remarkably, at the other two energies $\sqrt{s} \simeq M_{Z} \pm 3.5 \mathrm{GeV}, \Gamma_{Z} / M_{Z}$ suppression is still quite strong, more than factor $1 / 5$.

On the methodology side, although we are interested mainly in the IFI effect off the $Z$ peak, at $\sqrt{s} \simeq M_{Z} \pm$ $3.5 \mathrm{GeV}$, it is worth also keeping an eye on $\sqrt{s}=M_{Z}$ and energies far away from the resonance. This is simply because any technical problem or mistreatment of physics which may cause a small effect at $\sqrt{s} \simeq M_{Z} \pm 3.5 \mathrm{GeV}$ could be magnified there, making it easier to trace it back and eliminate. This is why we shall often compare our principal results with the results at $\sqrt{s}=M_{Z}$ and $\sqrt{s}=10 \mathrm{GeV}$.

\section{NEW CALCULATION IN THE SEMISOFT APPROXIMATION AND KKFoam MONTE CARLO}

As outlined in the Introduction, the aim of this section is to describe in detail all ingredients in the newly

\footnotetext{
${ }^{7}$ More precisely, $v=1-M_{\mu \mu}^{2} / s$. Here, we have temporarily used the ALEPH definition of $v$.
} 
developed KKFoam Monte Carlo which will be served in the next sections for validation of the KKMC predictions for $A_{\mathrm{FB}}(s)$ at a precision at least a factor of 10 better than in the past. KKFoam is not a true event generator because photon momenta are partly integrated out analytically. Nevertheless, it provides weighted MC events with explicit muon four-momenta; hence angular distributions of muons with an arbitrary cutoff on the total photon energy can be calculated.

In the following Sec. III A, the multiphoton matrix element (spin amplitudes) will be defined. In Secs. III B and III C the above matrix element will be squared, and spin summation and phase space integration will be done partly analytically. The IFI effect appears in the resulting muon angular distribution. The resulting formula involves a fourfold convolution over radiator functions of the initial state radiation (ISR), final state radiation (FSR) and two functions due to initial-final state interference (IFI). (Further analytic integration is not possible.)

In addition, in Sec. III D, the phase space integration is extended to the full phase space, and matching with the known $\mathcal{O}\left(\alpha^{1}\right)$ and $\mathcal{O}\left(\alpha^{1}\right)$ results for the ISR and FSR radiator functions is performed. The radiator functions are convoluted with the effective Born spin amplitudes in which EW corrections are included.

In Sec. IIIE, it is explained how the remaining 5-dimensional integration over the four radiator functions (ISR, FSR and $2 \times \mathrm{IFI}$ ) and the azimuthal angle $\theta$ of the muon is performed numerically using a Monte Carlo method. It is not trivial due to the presence of new types of singularities in the IFI radiator functions, different from the standard ones of the ISR and FSR radiator functions. The newly developed computer program KKFOam is new software tool, methodologically completely independent of KKMC, although it exploits some building blocks of KKMC, for instance the DIZET library of the EW corrections [28].

\section{A. Matrix element of multi-soft-photon emission in the semisoft approximation}

Let us consider the matrix element of the process

$$
\begin{aligned}
& e^{-}\left(p_{1}\right)+e^{+}\left(p_{2}\right) \rightarrow \mu^{-}\left(q_{1}\right)+\mu^{+}\left(q_{2}\right)+\gamma\left(k_{1}\right) \\
& \quad+\cdots+\gamma\left(k_{n}\right)
\end{aligned}
$$

near the $Z$ resonance in the soft photon limit. The standard kinematic variables $s=\left(p_{1}+p_{2}\right)^{2}, t=\left(q_{1}-p_{1}\right)^{2}, u=$ $\left(q_{2}-p_{1}\right)^{2}$ will be used. Around any narrow resonance, the notion of the soft photon limit has to be refined. In the framework of the standard Yennie-Frautschi-Suura (YFS) [25] soft photon resummation, one starts with all photons being very soft, i.e. $k_{i}^{0} \ll \Gamma_{Z} \ll \sqrt{s} / 2$. Near the resonance, however, it is worth considering a wider soft photon range, with $k_{i}^{0} \ll \sqrt{s} / 2$, but allowing photon energies comparable to or even greater than the resonance width $\Gamma_{Z}$. In the following, we shall refer to this regime as the semisoft approximation. Following the notation of Ref. [12], in the semisoft regime, the matrix element of our process reads as follows:

$$
\begin{aligned}
\mathscr{M}^{\mu_{1}, \mu_{2}, \ldots, \mu_{n}}\left(p_{i}, q_{j}, k_{l}\right)= & \sum_{V=\gamma, Z} \sum_{\mathscr{P}} e^{\alpha B_{4}^{V}\left(s_{I}, t, m_{\gamma}\right)} \prod_{i \in I} j_{I}^{\mu_{i}}\left(k_{i}\right) \\
& \times \prod_{r \in F} j_{F}^{\mu_{r}}\left(k_{r}\right) \mathcal{M}_{V}\left(s_{I}, t\right), \\
s_{I} & =P_{I}^{2}, \quad P_{I}=p_{1}+p_{2}-\sum_{i \in I} k_{j}, \\
j_{I}^{\mu}(k)= & e Q_{e}\left(\frac{p_{1}^{\mu}}{k p_{1}}-\frac{p_{2}^{\mu}}{k p_{2}}\right), \\
j_{F}^{\mu}(k)= & e Q_{f}\left(\frac{q_{1}^{\mu}}{k q_{1}}-\frac{q_{2}^{\mu}}{k q_{2}}\right), \\
\alpha B_{4}^{V}\left(s, t, m_{\gamma}\right) & =\alpha B_{4}\left(s, t, m_{\gamma}\right)+\alpha \Delta B_{4}^{V}\left(s, t, \bar{M}_{V}^{2}\right) .
\end{aligned}
$$

The above formula involves a sum over the set of $2^{n}$ partitions $\{\mathscr{P}\}=\{I, F\}^{n}$,

$$
\begin{aligned}
\{\mathscr{P}\}= & \{(I, I, I, \ldots, I),(F, I, I, \ldots, I),(I, F, I, \ldots, I), \\
& (F, F, I, \ldots, I), \ldots,(F, F, I, \ldots, F)\},
\end{aligned}
$$

of photons among the initial and final state. The meaning of the shorthand notation $i \in I$ is that $\prod_{i \in I}$ includes all photons with $\mathscr{P}_{i}=I$ and similarly $\mathscr{P}_{r}=F$ for $r \in F$.

The form factor $B_{4}\left(p_{i}, q_{i}, m_{\gamma}\right)$ is the standard one appearing in YFS resummation [25] for four charged particles in the scattering process. As stressed in Refs. [18-20], in the semisoft regime, an additional term in the form factor

$$
\begin{aligned}
\alpha \Delta B_{4}^{Z}\left(s, t, \bar{M}^{2}\right) & =-2 Q_{e} Q_{f} \frac{\alpha}{\pi} \ln \left(\frac{t}{u}\right) \ln \left(\frac{\bar{M}_{Z}^{2}-s}{\bar{M}_{Z}^{2}}\right), \\
\bar{M}^{2} & =M_{Z}^{2}-i M_{Z} \Gamma_{Z}, \quad \Delta B_{4}^{\gamma} \equiv 0,
\end{aligned}
$$

must be included, but only in the resonant component of the amplitude. For $\gamma$ exchange, only the standard $\alpha B_{4}$ of the YFS scheme is needed, and $\alpha B_{4}^{V}$ is not present. Most important is that, in the semisoft approximation, the energy argument of the resonance propagator in the Born matrix element $\mathcal{M}_{V}$ must be shifted by the total energy lost to initial state photons $j \in I,{ }^{8}$ because of its strong energy dependence. The same additional dependence on $s_{I}$ also enters into the form factor $\alpha B_{4}^{V}$. The summation over all partitions of $n$ photons between the initial and final state $\{I, F\}$ is mandatory in order to obey Bose-Einstein

\footnotetext{
${ }^{8}$ In the strict YFS soft limit this energy shift may be neglected. In the semisoft regime it could also be neglected for the $\gamma$ exchange part. For the sake of a better treatment of the collinear (mass) singularities, it is best to keep it everywhere.
} 
symmetry and gauge invariance. Fermion spinor indices are implicit in $\mathcal{M}_{V}$. The standard YFS virtual form factor $B_{4}$ is usually regularized with a photon mass $m_{\gamma}$. The mass regulator can be removed once the real and virtual calculations are combined.

In the framework of coherent exclusive exponentiation (CEEX) [12,22], the above matrix element represents a zeroth-order CEEX matrix element defined throughout the entire phase space, including hard photons. Higher orders are also defined in the CEEX scheme, and implemented for a finite number of the hard photons; see Ref. [12].

The same matrix element can be rewritten in a compact form using a generating functional formulation (MellinFourier transform):

$$
\begin{aligned}
& \mathscr{M}^{\mu_{1}, \mu_{2}, \ldots, \mu_{n}}\left(p_{i}, q_{j}, k_{1}, \ldots, k_{n}\right)=\sum_{V=\gamma, Z} \int \frac{d^{4} Q d^{4} x}{(2 \pi)^{4}} e^{i x \cdot(P-Q)} e^{\alpha B_{4}^{V}\left(Q^{2}, t, m_{\gamma}\right)}\left[\prod_{i=1}^{n} J^{\mu_{i}}\left(x, k_{i}\right)\right] \mathcal{M}_{V}\left(Q^{2}, t\right) \\
& J^{\mu}(x, k)=e^{-i k \cdot x} j_{I}^{\mu}(k)+j_{F}^{\mu}(k) .
\end{aligned}
$$

The corresponding total cross section reads:

$$
\begin{aligned}
\sigma(s)= & \frac{1}{\operatorname{flux}(s)} \sum_{n=0}^{\infty} \frac{1}{n !} \int \frac{d^{3} q_{1}}{q_{1}^{0}} \frac{d^{3} q_{2}}{q_{2}^{0}} \prod_{i=1}^{n} \int \frac{d^{3} k_{i}}{k_{i}^{0}} \delta\left(P-q_{1}-q_{2}-\sum_{i=1}^{n} k_{i}\right) \\
& \times \mathscr{M}^{\mu_{1}, \mu_{2}, \ldots, \mu_{n}}\left(p, q, k_{1}, \ldots, k_{n}\right)\left[\mathscr{M}_{\mu_{1}, \mu_{2}, \ldots, \mu_{n}}\left(p, q, k_{1}, \ldots, k_{n}\right)\right]^{*},
\end{aligned}
$$

where $P=p_{1}+p_{2}$. Note that in the above expression, the standard Lorentz invariant phase space integral extends over the entire phase space, as it would in the Monte Carlo implementation, i.e., energy conservation naturally limits photon energies from above. A cutoff on the total photon energy will be imposed later in our analytic calculations, in order to perform phase space integration analytically.

In the semisoft approximation, the matrix element of Eq. (5) is simple enough that in the absence of experimental cuts one can perform an analytic integration over photon angles and energies and sum explicitly over photon multiplicities. This is what we refer to as an analytic exponentiation.

The first important step will be reorganization of the multiphoton distributions keeping phase space integration untouched. Later on phase space integrations will be done in the semisoft regime and finally contributions from hard photon phase space will be reintroduced in the standard matching procedure at the level of partly integrated distributions.

\section{B. Reorganization of multiphoton distributions}

One may perform analytic reorganization of multiphoton distributions, a necessary first step in the analytic exponentiation, by means of a combinatorial reorganization of the sum over photons without using the generating functional (Mellin-Fourier transform) formulation of Eq. (8). This method was developed in Ref. [12], albeit for the resonant component only. Another alternative method of integration/summation over semisoft photons would employ a coherent states technique. This method was used, for instance, in Refs. [18,19]. Let us start from the generating functional form of Eq. (8), which was used in the original YFS paper [25], although it was applied there for the simpler nonresonant case. Of course, all three methods lead to an identical final result.

In the first step, let us introduce the usual eikonal factors for photon emission from the initial state, final state, and their interference:

$$
\begin{aligned}
S_{I}(k) & =-j_{I}(k) \cdot j_{I}(k), \quad S_{F}(k)=-j_{F}(k) \cdot j_{F}(k), \\
S_{X}(k) & =-j_{I}(k) \cdot j_{F}(k),
\end{aligned}
$$

and Fourier representations of the $\delta$-functions of the phase space: ${ }^{9}$

$$
\begin{aligned}
\sigma(s)= & \frac{1}{\operatorname{flux}(s)} \sum_{n=0}^{\infty} \frac{1}{n !} \sum_{V, V^{\prime}} \int \frac{d^{3} q_{1}}{q_{1}^{0}} \frac{d^{3} q_{2}}{q_{2}^{0}} \frac{d^{4} Q d^{4} x}{(2 \pi)^{4}} \frac{d^{4} Q^{\prime} d^{4} x^{\prime}}{(2 \pi)^{4}} e^{i x \cdot(P-Q)-i x^{\prime} \cdot\left(P-Q^{\prime}\right)} \frac{d^{4} y}{(2 \pi)^{4}} e^{i y \cdot\left(P-q_{1}-q_{2}\right)} \\
& \times \prod_{i=1}^{n} \int \frac{d^{3} k_{i}}{k_{i}^{0}}\left[e^{-i k_{i} \cdot\left(y+x-x^{\prime}\right)} S_{I}\left(k_{i}\right)+e^{-i k_{i} \cdot(y+x)} S_{X}\left(k_{i}\right)+e^{-i k_{i} \cdot\left(y-x^{\prime}\right)} S_{X}\left(k_{i}\right)+e^{-i k_{i} \cdot y} S_{F}\left(k_{i}\right)\right] \\
& \times \mathcal{M}_{V}(Q, t) \mathcal{M}_{V^{\prime}}^{*}\left(Q^{\prime}, t\right) e^{\alpha B_{4}^{V}\left(Q^{2}, t, m_{\gamma}\right)+\alpha B_{4}^{* V^{\prime}}\left(Q^{\prime 2}, t, m_{\gamma}\right)} .
\end{aligned}
$$

\footnotetext{
${ }^{9}$ The overall 4-momentum conservation $\delta^{(4)}$-function will be present implicitly in the next steps.
} 
In the above functional representation, the summation over photon multiplicities (exponentiation) is trivial: ${ }^{10}$

$$
\begin{aligned}
\sigma(s)= & \frac{1}{\operatorname{flux}(s)} \sum_{V, V^{\prime}} \int \frac{d^{3} q_{1}}{q_{1}^{0}} \frac{d^{3} q_{2}}{q_{2}^{0}} \frac{d^{4} Q d^{4} x}{(2 \pi)^{4}} \frac{d^{4} Q^{\prime} d^{4} x^{\prime}}{(2 \pi)^{4}} e^{i x \cdot(P-Q)-i x^{\prime} \cdot\left(P-Q^{\prime}\right)} \frac{d^{4} y}{(2 \pi)^{4}} e^{i y \cdot\left(P-q_{1}-q_{2}\right)} \\
& \times \exp \left\{\int \frac{d^{3} k}{k^{0}}\left[e^{-i k \cdot\left(y+x-x^{\prime}\right)} S_{I}(k)+e^{-i k \cdot(y+x)} S_{X}(k)+e^{-i k \cdot\left(y-x^{\prime}\right)} S_{X}(k)+e^{-i k \cdot y} S_{F}(k)\right]\right\} \\
& \times \exp \left\{\alpha B_{4}^{V}\left(Q^{2}, s, t\right)+\alpha\left(B_{4}^{V^{\prime}}\left(Q^{\prime 2}, s, t\right)\right)^{*}\right\} \mathcal{M}_{V}(Q, t) \mathcal{M}_{V^{\prime}}^{*}\left(Q^{\prime}, t\right) .
\end{aligned}
$$

The integrations over $x, x^{\prime}$ and $y$ can be reorganized in order to achieve a clear factorization into ISR, FSR, and IFI parts, as shown in Appendix A.

A slightly reorganized form of Eq. (A2) with $U^{\mu}$ representing the total photon momentum of pure FSR emission, $K^{\mu}$ representing the total momentum of pure ISR emission, and with $R^{\mu}$ and $R^{\prime \mu}$ aggregating IFI photons present in $\mathcal{M}_{V}$ and $\mathcal{M}_{V^{\prime}}^{*}$, correspondingly, reads as follows:

$$
\begin{aligned}
\sigma(s)= & \frac{1}{\text { flux }(s)} \int \frac{d^{3} q_{1}}{2 q_{1}^{0}} \frac{d^{3} q_{2}}{2 q_{2}^{0}} d^{4} K d^{4} R d^{4} R^{\prime} d^{4} U \delta^{4}\left(P-q_{1}-q_{2}-K-R-R^{\prime}-U\right) \\
& \times \int \frac{d^{4} z}{(2 \pi)^{4}} e^{i z \cdot K+\int \frac{d^{3} k}{k^{0}} e^{-i k \cdot z} S_{I}(k)} \int \frac{d^{4} u}{(2 \pi)^{4}} e^{i u \cdot R+\int \frac{d^{3} k}{k^{0}} e^{-i k \cdot u} S_{X}(k)} \times \int \frac{d^{4} u^{\prime}}{(2 \pi)^{4}} e^{i u^{\prime} \cdot R^{\prime}+\int \frac{d^{3} k}{k^{0}} e^{-i k \cdot u^{\prime}} S_{X}(k)} \\
& \int \frac{d^{4} y}{(2 \pi)^{4}} e^{i y \cdot U+\int \frac{d^{3} k}{k^{0}} e^{-i k \cdot y} S_{F}(k)} \sum_{V, V^{\prime}=\gamma, Z} \mathcal{M}_{V}(P-K-R) \mathcal{M}_{V^{\prime}}^{*}\left(P-K-R^{\prime}\right) \\
& \times \exp \left\{2 \alpha \Re B_{4}\left(s, t, m_{\gamma}\right)+\alpha \Delta B_{4}^{V}\left((P-K-R)^{2}\right)+\left(\alpha \Delta B_{4}^{V^{\prime}}\left(\left(P-K-R^{\prime}\right)^{2}\right)\right)^{*}\right\} ;
\end{aligned}
$$

see also the illustration in Fig. 6.

The role of the Mellin transform in the above algebra was merely to provide compact bookkeeping of the complicated sums in the multiphoton distributions, without any modification of the underlying phase space integration. At any step, we could go back to standard phase space without any cutoffs; for instance Eq. (13) can be rewritten as follows:

$$
\begin{aligned}
\sigma(s) & =\frac{1}{\text { flux }(s)} \int \frac{d^{3} q_{1}}{2 q_{1}^{0}} \frac{d^{3} q_{2}}{2 q_{2}^{0}} d^{4} K d^{4} R d^{4} R^{\prime} d^{4} U \delta^{4}\left(P-q_{1}-q_{2}-K-R-R^{\prime}-U\right) \\
& \times \sum_{n_{1}=0}^{\infty} \frac{1}{n_{1} !} \prod_{i_{1}=1}^{n_{1}} \frac{d^{3} k_{i_{1}}}{k_{i_{1}}^{0}} S_{I}\left(k_{i_{1}}\right) \delta_{K=\sum_{i_{1}=1}^{n_{1}} k_{i_{1}}} \sum_{n_{2}=0}^{\infty} \frac{1}{n_{2} !} \prod_{i_{2}=1}^{n_{2}} \frac{d^{3} k_{i_{2}}}{k_{i_{2}}^{0}} S_{X}\left(k_{i_{2}}\right) \delta_{R=\sum_{i_{2}=1}^{n_{1}} k_{i_{2}}} \sum_{n_{3}=0}^{\infty} \frac{1}{n_{3} !} \prod_{i_{3}=1}^{n_{3}} \frac{d^{3} k_{i_{3}}}{k_{i_{3}}^{0}} S_{X}\left(k_{i_{3}}\right) \delta_{R^{\prime}=\sum_{i_{3}=1}^{n_{3}} k_{i_{3}}} \\
& \times \sum_{n_{4}=0}^{\infty} \frac{1}{n_{4} !} \prod_{i_{4}=1}^{n_{4}} \frac{d^{3} k_{i_{4}}}{k_{i_{4}}^{0}} S_{F}\left(k_{i_{4}}\right) \delta_{U=\sum_{i_{4}=1}^{n_{4}} k_{i_{4}}} \sum_{V, V^{\prime}=\gamma, Z} \mathcal{M}_{V}(P-K-R) \mathcal{M}_{V^{\prime}}^{*}\left(P-K-R^{\prime}\right) \exp \left\{2 \alpha \Re B_{4}\left(s, t, m_{\gamma}\right)\right. \\
& \left.+\alpha \Delta B_{4}^{V}\left((P-K-R)^{2}\right)+\left(\alpha \Delta B_{4}^{V^{\prime}}\left(\left(P-K-R^{\prime}\right)^{2}\right)\right)^{*}\right\} .
\end{aligned}
$$

This is a generalization of Eq. (88) in Ref. [12], which was obtained there using pure combinatorics, without any use of the Mellin-Fourier transform. Both virtual and real photon integrals are IR-regularized using finite photon mass $m_{\gamma}$.

Another advantage of the compact Eq. (13) is that by means of adding and subtracting

$$
\int_{k^{0} \leq E} \frac{d^{3} k}{k^{0}}\left[S_{I}(k)+2 S_{X}(k)+S_{F}(k)\right], \quad E=\frac{\sqrt{s}}{2},
$$

in the form-factor exponent, we obtain a manifestly IR-finite expression [25]:

\footnotetext{
${ }^{10} \mathrm{Both}$ the virtual functions $B_{4}^{V}$ and real emission integrals over $S$-factors are regularized temporarily using a small photon mass $m_{\gamma}$, which will cancel in the final result.
} 


$$
\begin{aligned}
\sigma(s)= & \frac{1}{\operatorname{flux}(s)} \int \frac{d^{3} q_{1}}{2 q_{1}^{0}} \frac{d^{3} q_{2}}{2 q_{2}^{0}} d^{4} K d^{4} R d^{4} R^{\prime} d^{4} U \delta^{4}\left(P-q_{1}-q_{2}-K-R-R^{\prime}-U\right) \\
& \times \int \frac{d^{4} z}{(2 \pi)^{4}} e^{i z \cdot K+\int \frac{d^{3} k}{k^{0}}\left[e^{-i k \cdot z}-\theta\left(k^{0}<E\right)\right] S_{I}(k)} \int \frac{d^{4} u}{(2 \pi)^{4}} e^{i u \cdot R+\int \frac{d^{3} k}{k^{0}}\left[e^{-i k \cdot u}-\theta\left(k^{0}<E\right)\right] S_{X}(k)} \int \frac{d^{4} u^{\prime}}{(2 \pi)^{4}} e^{i u^{\prime} \cdot R^{\prime}+\int \frac{d^{3} k}{k^{0}}\left[e^{-i k \cdot u^{\prime}}-\theta\left(k^{0}<E\right)\right] S_{X}(k)} \\
& \times \int \frac{d^{4} y}{(2 \pi)^{4}} e^{i y \cdot U+\int \frac{d^{3} k}{k^{0}}\left[e^{-i k \cdot y}-\theta\left(k^{0}<E\right)\right] S_{F}(k)} \sum_{V, V^{\prime}=\gamma, Z} \mathcal{M}_{V}(P-K-R) \mathcal{M}_{V^{\prime}}^{*}\left(P-K-R^{\prime}\right), \exp \left\{Y\left(p_{i}, q_{i}\right)\right. \\
& \left.+\alpha \Delta B_{4}^{V}\left((P-K-R)^{2}\right)+\left(\alpha \Delta B_{4}^{V^{\prime}}\left(\left(P-K-R^{\prime}\right)^{2}\right)\right)^{*}\right\},
\end{aligned}
$$

where $\left(e^{-i k \cdot y}-1\right) / k^{0}$ is IR-finite for $k^{0} \rightarrow 0$ and the classic YFS form factor

$$
Y\left(p_{i}, q_{i}\right)=2 \alpha \Re B_{4}\left(s, t, m_{\gamma}\right)+\int_{k^{0} \leq E} \frac{d^{3} k}{k^{0}}\left[S_{I}(k)+2 S_{X}(k)+S_{F}(k)\right]
$$

is also finite in the $m_{\gamma} \rightarrow 0$ limit.

Reintroducing an IR cutoff $\varepsilon$ on the real photon energies, Eq. (16) can be also rewritten in the following equivalent form with the standard phase space integration and without any Mellin transforms [31]:

$$
\begin{aligned}
\sigma(s)= & \frac{1}{\text { flux }(s)} \int \frac{d^{3} q_{1}}{2 q_{1}^{0}} \frac{d^{3} q_{2}}{2 q_{2}^{0}} d^{4} K d^{4} R d^{4} R^{\prime} d^{4} U \delta^{4}\left(P-q_{1}-q_{2}-K-R-R^{\prime}-U\right) \sum_{n_{1}=0}^{\infty} \frac{1}{n_{1} !} \int_{k_{i_{1}}^{0}>\varepsilon} \prod_{i_{1}=1}^{n_{1}} \frac{d^{3} k_{i_{1}}}{k_{i_{1}}^{0}} S_{I}\left(k_{i_{1}}\right) \delta_{K=\sum_{i_{1}=1}^{n_{1}} k_{i_{1}}} \\
& \times \sum_{n_{2}=0}^{\infty} \frac{1}{n_{2} !} \int_{k_{i_{2}}^{0}>\varepsilon} \prod_{i_{2}=1}^{n_{2}} \frac{d^{3} k_{i_{2}}}{k_{i_{2}}^{0}} S_{X}\left(k_{i_{2}}\right) \delta_{R=\sum_{i_{2}=1}^{n_{1}} k_{i_{2}}} \sum_{n_{3}=0}^{\infty} \frac{1}{n_{3} !} \int_{k_{i_{3}}^{0}>\varepsilon} \prod_{i_{3}=1}^{n_{3}} \frac{d^{3} k_{i_{3}}}{k_{i_{3}}^{0}} S_{X}\left(k_{i_{3}}\right) \delta_{R^{\prime}=\sum_{i_{3}=1}^{n_{3}} k_{i_{3}}} \\
& \times \sum_{n_{4}=0}^{\infty} \frac{1}{n_{4} !} \int_{k_{i_{3}}^{0}>\varepsilon} \prod_{i_{4}=1}^{n_{4}} \frac{d^{3} k_{i_{4}}}{k_{i_{4}}^{0}} S_{F}\left(k_{i_{4}}\right) \delta_{U=\sum_{i_{4}=1}^{n_{4}} k_{i_{4}}} \exp \left(-\int_{\varepsilon<k^{0}<E} \frac{d^{3} k}{k^{0}} S(k)\right) \sum_{V, V^{\prime}=\gamma, Z} \mathcal{M}_{V}(P-K-R) \mathcal{M}_{V^{\prime}}^{*}\left(P-K-R^{\prime}\right) \\
& \times \exp \left\{Y\left(p_{i}, q_{i}\right)+\alpha \Delta B_{4}^{V}\left((P-K-R)^{2}\right)+\left(\alpha \Delta B_{4}^{V^{\prime}}\left(\left(P-K-R^{\prime}\right)^{2}\right)\right)^{*}\right\}
\end{aligned}
$$

The phase space integration in the above formula cannot be performed analytically. (It is done numerically without any approximation in the KKMC program.) In the following, this phase space integration will be done analytically in the semisoft approximation.

\section{Analytic integration over photon momenta}

In the next step, we shall integrate over the photon angles in Eq. (16), introducing the cutoff $E_{\max }=v_{\max } \frac{1}{2} s^{1 / 2}$,

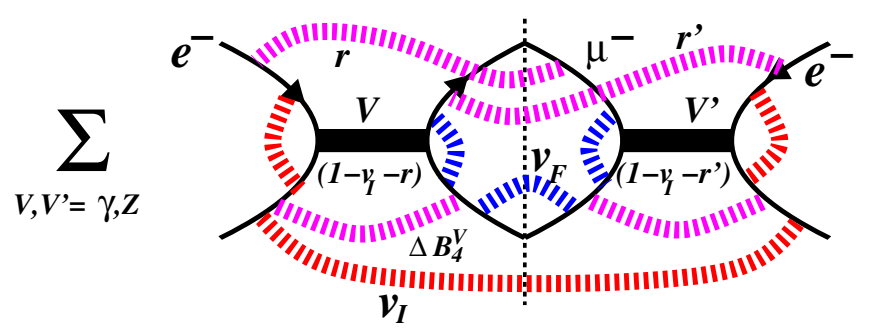

FIG. 6. Exponentiated multiple photon emission from initial and final fermions including ISR, FSR, and IFI in the resonant process, as in Eq. (13). Dashed lines represent multiple real and/ or virtual photons. $v_{\max } \ll 1$, on the total photon energy in order to simplify the phase space integral, and staying within the semisoft approximation for the multiphoton distributions, as in Eq. (16) and Eq. (18).

Let us show how it is done for the initial state part of this multiphoton integral. In the semisoft photon limit, the integrand of Eq. (16) has no dependence on the spatial components of $K$ outside of the $e^{i z \cdot K}$ factor. Typically, the Born matrix element and the resonant form factor have a dependence on $K^{0}$ through

$$
\begin{aligned}
(P-K-R)^{2} & =P^{2}-2 P \cdot(K+R)+(K+R)^{2} \\
& \simeq s-2 \sqrt{s}\left(K^{0}+R^{0}\right),
\end{aligned}
$$

but no dependence on the spatial components $\vec{K}$. Thus, the integral over $\vec{K}$ yields a factor $\delta^{3}(\vec{z})$ and leads to ${ }^{11}$

\footnotetext{
${ }^{11}$ See also Eq. (36) in the next subsection for versions without a Mellin transform.
} 


$$
\begin{aligned}
& \int \frac{d^{4} K d^{4} z}{(2 \pi)^{4}} e^{i z \cdot K+\int \frac{d^{3} k}{k^{0}} S_{I}(k)\left[e^{-i k \cdot z}-\theta_{k^{0}<E}\right]}
\end{aligned}
$$

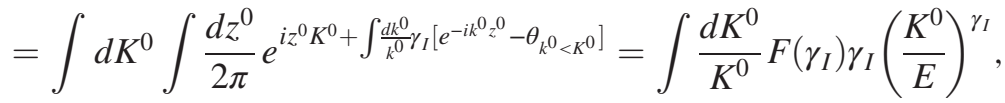

where the integration over photon angles resulted in

$$
\gamma_{I}=\gamma_{I}(s)=\int \frac{d^{3} k}{k^{0}} S_{I}(k) \delta\left(2 k^{0} / \sqrt{s}-1\right) .
$$

The subtle point is that the elimination of $\int d^{3} \vec{K} \delta^{3}\left(\vec{K}-\sum_{i=1}^{n} \vec{k}_{i}\right)$ implies that we keep $\vec{K}=\sum_{i=1}^{n} \vec{k}_{i}$ everywhere in the entire integrand. Note that in KKMC, the above "recoil effect" in the Born matrix element and phase space integral is taken into account correctly for hard photons as well. The function

$$
F(\gamma) \equiv \frac{\exp \left(-\gamma C_{E}\right)}{\Gamma(1+\gamma)}
$$

is well known from YFS work (Eq. (2.44) in Ref. [25]) and is due to the competition of soft real photons for the available fixed total energy.

Similarly, we are able to integrate over FSR and IFI photons:

$$
\begin{aligned}
& \int d^{4} U \frac{d^{4} y}{(2 \pi)^{4}} e^{i y \cdot U+\int \frac{d^{3} k}{k^{0}} S_{F}(k)\left[e^{-i k \cdot y}-\theta_{k^{0}<E}\right]}=\int \frac{d U^{0}}{U^{0}} \gamma_{F}\left(\frac{K^{0}}{E}\right)^{\gamma_{F}} F\left(\gamma_{F}\right), \\
& \int d^{4} R \frac{d^{4} u}{(2 \pi)^{4}} e^{i u \cdot R+\int \frac{d^{3} k}{k^{0}} S_{X}(k)\left[e^{-i k \cdot u}-\theta_{k^{0}<E}\right]}=\int \frac{d R^{0}}{R^{0}} \gamma_{X}\left(\frac{K^{0}}{E}\right)^{\gamma_{X}} F\left(\gamma_{X}\right), \\
& \int d^{4} R^{\prime} \frac{d^{4} u^{\prime}}{(2 \pi)^{4}} e^{i u^{\prime} \cdot R^{\prime}+\int \frac{d^{3} k}{k^{0}} S_{X}(k)\left[e^{-i k \cdot u^{\prime}}-\theta_{k^{0}<E}\right]}=\int \frac{d R^{\prime 0}}{R^{\prime 0}} \gamma_{X}\left(\frac{K^{0}}{E}\right)^{\gamma_{X}} F\left(\gamma_{X}\right),
\end{aligned}
$$

where

$$
\begin{aligned}
\gamma_{F}=\gamma_{F}(s) & =\int \frac{d^{3} k}{k^{0}} S_{F}(k) \delta\left(2 k^{0} / \sqrt{s}-1\right), \\
\gamma_{X}=\gamma_{X}(\cos \theta) & =\int \frac{d^{3} k}{k^{0}} S_{X}(k) \delta\left(2 k^{0} / \sqrt{s}-1\right),
\end{aligned}
$$

and $\theta$ is the angle the between the momenta $p_{1}$ of $e^{-}$and $q_{1}$ of $\mu^{-}$.

Inserting all the above into Eq. (16), we finally obtain a compact elegant formula:

$$
\begin{aligned}
\sigma\left(s, v_{\max }\right)= & \frac{3 \sigma_{0}(s)}{8} \sum_{V, V^{\prime}} \int_{0}^{1} d v_{I} d v_{F} d r d r^{\prime} \int \frac{d \cos \theta d \phi}{2} \theta\left(v_{\max }-v_{I}-r-r^{\prime}-v_{F}\right) \\
& \times \rho\left(\gamma_{I}, v_{I}\right) \rho\left(\gamma_{X}, r\right) \rho\left(\gamma_{X}, r^{\prime}\right) \rho\left(\gamma_{F}, v_{F}\right) e^{Y\left(p_{i}, q_{i}\right)} \\
& \times \frac{1}{4} \sum_{\varepsilon \tau} \mathfrak{R}\left\{e^{\alpha \Delta B_{4}^{V}\left(s\left(1-v_{I}-r\right)\right)} \mathfrak{M}_{\varepsilon \tau}^{V}\left(v_{I}+r, c\right)\left[e^{\alpha \Delta B_{4}^{V^{\prime}}\left(s\left(1-v_{I}-r^{\prime}\right)\right)} \mathfrak{M}_{\varepsilon \tau}^{V^{\prime}}\left(v_{I}+r^{\prime}, c\right)\right]^{*}\right\},
\end{aligned}
$$

where the Born spin amplitudes of Appendix $\mathrm{C}$ are used and we define

$$
\rho(\gamma, v)=F(\gamma) \gamma v^{\gamma-1}, \quad v_{I}=\frac{2 K^{0}}{\sqrt{s}}, \quad r=\ln \frac{2 R^{0}}{\sqrt{s}}, \quad r^{\prime}=\ln \frac{2 R^{\prime 0}}{\sqrt{s}}, \quad v_{F}=\frac{2 U^{0}}{\sqrt{s}} .
$$

The appearance of the real part $\Re\left[\mathcal{M}_{V} \mathcal{M}_{V^{\prime}}^{*}\right]$ has resulted from symmetrization over $r$ and $r^{\prime}$. The overall structure of the above integral is illustrated in Fig. 6. 
Note that the YFS function $\rho(\gamma, v)$ obeys the following nice convolution rule (related to the fact that it represents a Markovian process):

$$
\int d v_{1} d v_{2} \delta\left(v-v_{1}-v_{2}\right) \rho\left(\gamma_{1}, v_{1}\right) \rho\left(\gamma_{2}, v_{2}\right)=\rho\left(\gamma_{1}+\gamma_{2}, v\right),
$$

but this feature cannot be exploited to simplify the integral of Eq. (25), because of the peculiar dependence of the matrix element on $r$ and $r^{\prime}$. Let us stress that the double convolution over ISR photons, separately for the Born amplitude and its conjugate seen in Eq. (25), is the landmark feature of the semisoft exponentiation pioneered in Refs. $[18,19]$ and implemented in KKMC.

\section{Matching of analytic exponentiation with fixed orders}

Any matching of analytic exponentiation with fixedorder calculations must address the inclusion of the hard photon phase space beyond the semisoft regime represented in Eq. (25) with the cutoff $v_{\max } \ll 1$ on the total photon energy. The above matching will follow past examples in Refs. [10,12,32]. It will result in the formula valid for $0<v_{\max } \leq 1$.

In order to match analytic exponentiation with known analytic $\mathcal{O}\left(\alpha^{1,2,3}\right)$ QED results for ISR and FSR and compare with KKMC over the entire phase space, let us extrapolate the formula of Eq. (25) beyond the semisoft regime to the entire range of the variable

$$
v=1-M_{\mu \mu}^{2} / s, \quad v \in(0,1),
$$

replacing soft photon approximation

$$
v=v_{I}+v_{F}+r+r^{\prime}
$$

with a multiplicative ansatz guided by the collinear kinematics,

$$
1-v=\left(1-v_{I}\right)\left(1-v_{F}\right)(1-r)\left(1-r^{\prime}\right) .
$$

With this ansatz, Eq. (25) takes the form

$$
\begin{aligned}
\sigma^{(0)}\left(s, v_{\max }\right)= & \frac{3 \sigma_{0}(s)}{8} \sum_{V, V^{\prime}} \int d v d v_{I} d v_{F} d r d r^{\prime} \delta_{1-v=\left(1-v_{I}\right)\left(1-v_{F}\right)(1-r)\left(1-r^{\prime}\right)} \theta_{v_{\max }>v} \\
& \times \int \frac{d \cos \theta d \phi}{2} \rho\left(\gamma_{I}(s), v_{I}\right) \rho\left(\gamma_{F}\left(s\left(1-v_{I}\right)\left(1-v_{F}\right)\right), v_{F}\right) \rho\left(\gamma_{X}(c), r\right) \rho\left(\gamma_{X}(c), r^{\prime}\right) e^{Y\left(p_{i}, q_{i}\right)} \\
& \times \frac{1}{4} \sum_{\varepsilon \tau} \Re\left\{e^{\alpha \Delta B_{4}^{V}\left(s\left(1-v_{I}\right)(1-r)\right)} \mathfrak{M}_{\varepsilon \tau}^{V}\left(1-\left(1-v_{I}\right)(1-r), c\right)\right. \\
& \left.\times\left[e^{\alpha \Delta B_{4}^{V^{\prime}}\left(s\left(1-v_{I}\right)\left(1-r^{\prime}\right)\right)} \mathfrak{M}_{\varepsilon \tau}^{V^{\prime}}\left(1-\left(1-v_{I}\right)\left(1-r^{\prime}\right), c\right)\right]^{*}\right\},
\end{aligned}
$$

where $c=\cos \theta$ and we have inserted also the Born spin amplitudes of Appendix C, From now on we may use $0<v_{\max } \leq 1$.

In the numerical comparison of the above $\mathcal{O}\left(\alpha^{0}\right)_{\exp }$ formula with KKMC, it is worth including numerically significant $\mathcal{O}\left(\alpha^{2}\right)$ contributions from the trivial phase integration. It was shown in Ref. [12] [see Eq. (206) there] that the following substitution does the job:

$$
\begin{aligned}
\rho\left(\gamma_{I}, v_{I}\right) \rightarrow & \rho_{I}^{(0)}\left(\gamma_{I}, v_{I}\right)=\rho\left(\gamma_{I}, v_{I}\right) \exp \left[\frac{1}{4} \gamma_{I}+\frac{\alpha}{\pi}\left(-\frac{1}{2}+\frac{\pi^{2}}{3}\right)\right]\left[1-\frac{1}{4} \gamma_{I} \ln \left(1-v_{I}\right)\right], \\
\rho\left(\gamma_{F}, v_{F}\right) \rightarrow & \rho_{F}^{(0)}\left(\gamma_{F}, v_{F}\right)=\rho\left(\gamma_{F}, v_{F}\right) \\
& \times \exp \left[\frac{1}{4} \gamma_{I}+\frac{\alpha}{\pi}\left(-\frac{1}{2}+\frac{\pi^{2}}{3}\right)-\frac{\gamma_{F}}{2} \ln \left(1-v_{F}\right)\right]\left[1-\frac{1}{4} \gamma_{F} \ln \left(1-v_{F}\right)\right],
\end{aligned}
$$

where $\gamma_{F}=\gamma_{F}\left(s\left(1-v_{I}\right)\left(1-v_{F}\right)\right) .^{12}$

In order to compare with $\mathcal{O}\left(\alpha^{2}\right) \mathrm{KKMC}$ calculations [including non-IR contributions of IFI up to $\mathcal{O}\left(\alpha^{1}\right)$ ] it is also quite easy to upgrade the ISR and FSR radiator functions in Eqs. (32) to $\mathcal{O}\left(\alpha^{2}\right)$ :

\footnotetext{
${ }^{12}$ We could also use $\gamma_{F}=\gamma_{F}(s(1-v))$, but we have checked that it leads to the same numerical results.
} 


$$
\begin{aligned}
\rho\left(\gamma_{I}, v_{I}\right) \rightarrow & \rho_{I}^{(2)}\left(\gamma_{I}, v_{I}\right)=\rho\left(\gamma_{I}, v_{I}\right) \exp \left[\frac{1}{4} \gamma_{I}+\frac{\alpha}{\pi}\left(-\frac{1}{2}+\frac{\pi^{2}}{3}\right)\right]\left[1+\frac{\gamma_{I}}{4}+\frac{\gamma_{I}^{2}}{8}\right. \\
& \left.+v_{I}\left(-1+\frac{v_{I}}{2}\right)+\gamma_{I}\left(-\frac{v_{I}}{2}-\frac{1+3\left(1-v_{I}\right)^{2}}{4} \ln \left(1-v_{I}\right)\right)\right], \\
\rho\left(\gamma_{F}, v_{F}\right) \rightarrow & \rho_{F}^{(2)}\left(\gamma_{F}, v_{F}\right)=\rho\left(\gamma_{F}, v_{F}\right) \exp \left[\frac{1}{4} \gamma_{I}+\frac{\alpha}{\pi}\left(-\frac{1}{2}+\frac{\pi^{2}}{3}\right)-\frac{\gamma_{F}}{2} \ln \left(1-v_{F}\right)\right] \\
& \times\left[1+\frac{\gamma_{F}}{4}+\frac{\gamma_{F}^{2}}{8}+v_{F}\left(-1+\frac{v_{F}}{2}\right)+\gamma_{F}\left(-\frac{v_{F}}{2}+\frac{v_{F}\left(2-v_{F}\right)}{8} \ln \left(1-v_{I}\right)\right)\right] ;
\end{aligned}
$$

see Tables I and II in Ref. [12].

The resulting ISR + FSR + IFI formula of Ref. [29] with all the above upgrades of ISR and FSR radiator functions [with the resummation of $\left.\ln \left(\Gamma_{Z} / M_{Z}\right)\right]$ is now ready for the MC implementation.

We are also going to implement the following formula in which IFI is completely neglected:

$$
\begin{aligned}
& \sigma_{\mathrm{no} I \mathrm{II}}^{(0)}\left(s, v_{\max }\right) \\
& =\frac{3 \sigma_{0}(s)}{8} \int d v d v_{I} d v_{F} \delta_{1-v=\left(1-v_{I}\right)\left(1-v_{F}\right)} \theta_{v_{\max }>v} \\
& \quad \times \int \frac{d \cos \theta d \phi}{2} \rho\left(\gamma_{I}(s), v_{I}\right) \rho\left(\gamma_{F}\left(s\left(1-v_{I}\right)\left(1-v_{F}\right)\right), v_{F}\right) \\
& \quad \times e^{Y\left(p_{i}, q_{i}\right)} \frac{1}{4} \sum_{\varepsilon \tau}\left|\mathfrak{M}_{\varepsilon \tau}\left(v_{I}, c\right)\right|^{2}
\end{aligned}
$$

In the final push towards inclusion of as many known fixed order results as possible into the analytic exponentiation formula, we include the complete $\mathcal{O}\left(\alpha^{1}\right)$ virtual IFI contributions. This amounts to adding the non-IR parts of the $\gamma \gamma$ and $\gamma Z$ box diagrams explicitly provided in Eqs. (C12) in Appendix $\mathrm{C}$ to the Born spin amplitudes:

$$
\begin{aligned}
\mathfrak{M}_{\varepsilon \tau}(s, t) \rightarrow & \mathfrak{M}_{\varepsilon \tau}(s, t)+\mathfrak{M}_{\varepsilon \tau}^{\{\gamma \gamma\}}\left(s, t, m_{\gamma}\right)+\mathfrak{M}_{\varepsilon \tau}^{\{\gamma Z\}}\left(s, t, m_{\gamma}\right) \\
& -2 \alpha B_{4}\left(s, t, m_{\gamma}\right) \mathfrak{M}_{\varepsilon \tau}(s, t) \\
& -\alpha \Delta B_{4}^{Z}(s, t) \mathfrak{M}_{\varepsilon \tau}^{Z}(s, t)
\end{aligned}
$$

This is done in the framework of the standard YFS-inspired reorganization of the IR singularities, the same way as in the CEEX matrix element of KKMC, without any danger of double counting. The additional subtraction of $\alpha \Delta B_{4}^{V}$ prevents double counting with the resummation/exponentiation of this term in the semisoft regime.

In addition electroweak and QCD corrections are also included in coupling constants of Born amplitudes, the same way as in KKMC. Both KKMC and KKFoam use the DIZET library of $\mathcal{O}\left(\alpha^{1}\right)$ EW corrections [28] [including some of $\mathcal{O}\left(\alpha^{2}\right)$ ], and the method in which EW corrections are inserted into Born-like parts of the spin amplitudes in
KKMC is essentially the same as in ZFITTER [13]. It is described in Eqs. (21-25) of Ref. [22].

Finally, with all the above changes due to matching with $\mathcal{O}\left(\alpha^{1}\right)$ and $\mathcal{O}\left(\alpha^{2}\right)$ known fixed-order corrections, we are now ready to implement the results of analytic exponentiation of Eqs. (29) and (32) with radiator functions of Eq. (30), (31) and box insertions, using the Monte Carlo method.

Coming back to the extension of the phase space in Eqs. (29) and (32) we see that it has now a well defined meaning: for IFI switched off these formulas coincide with the well known QED convolution formulas for the total cross section $[10,12,32]$ including hard photons. However, for the angular distributions Eq. (32) is not able to reproduce exactly the $\mathcal{O}\left(\alpha^{1,2}\right)$ angular distribution beyond the soft limit. On the other hand, from the analysis of Ref. [33] it is known that it reproduces numerically very well $\mathcal{O}\left(\alpha^{1,2}\right)$ MC results without IFI near the $\mathrm{Z}$ resonance for $A_{\mathrm{FB}}$ calculated practically for any choices on the muon angle, within realistic cutoffs including hard photon emissions. Unfortunately, the known nonsoft $\mathcal{O}\left(\alpha^{1}\right)$ IFI contributions to angular distributions, see Ref. [8] and Appendix C, cannot be reproduced exactly ${ }^{13}$ by Eqs. (29), (32). The main aim of Eqs. (29), (32) with radiator functions of Eqs. (30), (31) is to test the soft limit of IFI implementation in KKMC in the presence of $\mathcal{O}\left(\alpha^{2}\right)$ ISR, FSR and $\mathcal{O}\left(\alpha^{1}\right)$ EW corrections.

How different are the above analytic resummations of the IFI effect in the semisoft approximation from the known similar calculations in the literature [18-20]? Although the starting point in terms of multiphoton amplitudes is the same, semisoft resummation in Ref. [18] exploits techniques of coherent states and Mellin transform for dealing with multiple sums over photons and phase space integration, while our approach is based on the straightforward combinatorics and direct phase space integration. References [18-20] attempt to do final phase space integration analytically, while in our approach we perform them numerically, gaining more

\footnotetext{
${ }^{13}$ It would cost adding two extra phase space integration variables in KKFoam in order to complete $\mathcal{O}\left(\alpha^{1}\right)$.
} 
flexibility in the matching with finite order results and in numerical comparisons with KKMC.

As a parenthetical remark, let us remark that one may try to do some extra ad hoc simplifications, strictly speaking not justified in the semisoft regime, which may have some practical advantages in the parametrization of the $\mathrm{MC}$ results or data. One example is the following variant of Eq. (29) which implements IFI in the approximate form: ${ }^{14}$

$$
\begin{aligned}
\sigma^{(0)}\left(s, v_{\max }\right)= & \frac{3 \sigma_{0}(s)}{8} \int d v d v_{I} d v_{F} d u \delta_{1-v=\left(1-v_{I}\right)\left(1-v_{F}\right)(1-u)} \theta_{v_{\max }>v} \\
& \times \int \frac{d \cos \theta d \phi}{2} \rho\left(\gamma_{I}(s), v_{I}\right) \rho\left(\gamma_{F}\left(s\left(1-v_{I}\right)\left(1-v_{F}\right)\right), v_{F}\right) \rho\left(2 \gamma_{X}(c), u\right) e^{Y(s, c)} \\
& \times \frac{1}{4} \sum_{\varepsilon \tau}\left|\sum_{V} e^{2 \alpha \Delta B_{4}^{V}\left(s\left(1-v_{I}\right)(1-u)\right)} \mathfrak{M}_{\varepsilon \tau}^{V}\left(c, 1-\left(1-v_{I}\right)(1-u), c\right)\right|^{2} .
\end{aligned}
$$

The simplification is due to neglecting $r$ and $r^{\prime}$ dependence in the Born matrix element and keeping the integration over $u=r+r^{\prime}$. The quality of the above approximation can only be judged using numerical tests.

\section{E. Numerical integration methodology}

Our aim is to perform numerically the 5-dimensional and 3-dimensional integrals in Eqs. (29) and (32) using the Monte Carlo integrator FOAM [29,30]. This is not quite trivial because the integrand of Eqs. (29) and (32) is singular and nonpositive. Singularities due to $\rho_{I}$ of ISR and $\rho_{F}$ of FSR can be easily eliminated with the following simple mapping of variables: ${ }^{15}$

$$
v=x_{\max } y_{1}^{1 / \gamma_{I}}, \quad u=x_{\max } y_{2}^{1 / \gamma_{I}}, \quad y_{i} \in(0,1) .
$$

The variable $x_{\max }=0.999 \ldots$ is a technical cutoff introduced to avoid numerical instabilities near $v=1$. The main problem is the integration over the two more strongly singular and nonpositive $\rho_{X}$ factors. This occurs when

$$
\gamma_{X}(\theta)=2 Q_{e} Q_{f} \frac{\alpha}{\pi} \ln \left(\frac{1-\cos \theta}{1+\cos \theta}\right)
$$

becomes negative: $\gamma_{X}(\theta)=-\beta<0$ in the forward hemisphere, where $\cos \theta>0$.

In fact, one may think that in such a case the integral of Eq. (25) does not make sense at all, because the singularity $r^{\gamma X^{-1}}=r^{-\beta-1}$ from $\rho_{X}$ is even not integrable! However, a closer examination of the multiphoton integral which has led to $\rho_{X}\left(r, \gamma_{X}(\theta)\right)$ reveals that the original distribution is in fact regularized with the familiar plusprescription $(\ldots)_{+}$.

In order to understand the problem better, it is worth examining the generic YFS multiphoton integral:

$$
\begin{aligned}
& \int_{K^{0}<E} \frac{d^{4} K d^{4} z}{(2 \pi)^{4}} e^{i z \cdot K+\int \frac{d^{3} k}{k^{0}} S(k)\left[e^{-i k \cdot z}-\theta_{k^{0}<E}\right]} \\
& =\int_{K^{0}<E} d^{4} K e^{-\int \frac{d^{3} k}{k^{0}} S(k) \theta_{\varepsilon<k^{0}<E}} \sum_{n=0}^{\infty} \frac{1}{n !} \prod_{i=1}^{n} \int_{\varepsilon<k^{0}<E} \frac{d^{3} k_{i}}{k_{i}^{0}} S\left(k_{i}\right) \delta^{4}\left(K-\sum_{i=1}^{n} k_{i}\right) \\
& =\int_{0}^{E} d K^{0} e^{-\int \gamma \frac{d k^{0}}{k^{0}} \theta_{\varepsilon<k^{0}<K^{0}}} \sum_{n=0}^{\infty} \frac{1}{n !} \prod_{i=1}^{n} \int_{\varepsilon<k^{0}<K^{0}} \gamma \frac{d k_{i}^{0}}{k_{i}^{0}} \delta\left(K^{0}-\sum_{i=1}^{n} k_{i}^{0}\right) \\
& =\int_{0}^{E} d K^{0} \int \frac{d z}{2 \pi} e^{i z K^{0}+\int \frac{d k^{0}}{k^{0}} \gamma\left[e^{-i k^{0} z}-\theta_{k^{0}<k^{0}}\right]} \\
& =\int_{0}^{E} \frac{d K^{0}}{K^{0}} \gamma F(\gamma)\left(\frac{K^{0}}{E}\right)^{\gamma}=\int_{0}^{1} d v \gamma v^{\gamma-1} F(\gamma)=\int_{0}^{1} d v \rho(\gamma, v)=F(\gamma) .
\end{aligned}
$$

\footnotetext{
${ }^{14}$ There are more variants of this formula, for instance setting $u=0$ in Born matrix element and form factor, etc.

${ }^{15}$ FOAM can cope with these singularities even without such a mapping.
} 
It is easy to check that the above integral is always finite and well defined for any choice of $S=S_{I}, S_{F}, S_{X}$, even for negative $S$ and for negative $\gamma$ ! Obviously, for $\gamma>0$, the singularity $v^{\gamma-1}$ is integrable and does not require any regulation. Closer inspection of Eq. (36) with an explicit IR regulator $\epsilon \ll 1$ reveals that for any $\gamma$, including $\gamma=-\beta<0$, the following holds:

$$
\begin{aligned}
\rho(\gamma, v)= & e^{-\int \gamma^{d k^{0}} \theta^{0}} \theta_{\varepsilon<k^{0}<k^{0}} \sum_{n=0}^{\infty} \frac{1}{n !} \prod_{i=1}^{n} \int_{\varepsilon<k^{0}<K^{0}} \gamma \frac{d k_{i}^{0}}{k_{i}^{0}} \\
& \times \delta\left(K^{0}-\sum_{i=1}^{n} k_{i}^{0}\right) \\
= & \delta(v) F(\gamma)\left[1-\int_{\varepsilon}^{1} d v^{\prime} \gamma v^{\prime \gamma-1}\right] \\
& +\theta(v-\varepsilon) F(\gamma) \gamma v^{\gamma-1}=F(\gamma)\left[\delta(v)+\left(\gamma v^{\gamma-1}\right)_{+}\right] .
\end{aligned}
$$

The standard plus prescription can be formulated either in a regulator-independent way

$$
\int_{0}^{1} d v \phi(v)\left(\gamma v^{\gamma-1}\right)_{+}=\int_{0}^{1} d v[\phi(v)-\phi(0)] \gamma v^{\gamma-1}
$$

or with an explicit regulator $\varepsilon \ll 1$

$$
\begin{aligned}
\left(\gamma v^{\gamma-1}\right)_{+} & =\gamma v^{\gamma-1} \theta(v-\varepsilon)-\delta(v) \int_{\varepsilon}^{1} d v^{\prime} \gamma v^{\prime \gamma-1} \\
& =\gamma v^{\gamma-1} \theta(v-\varepsilon)-\delta(v)\left[1-\varepsilon^{\gamma}\right] .
\end{aligned}
$$

Of course, for $\gamma>0$, it becomes simpler, because for $\varepsilon \rightarrow 0$, we get $\left(\gamma v^{\gamma-1}\right)_{+} \rightarrow \gamma v^{\gamma-1}-\delta(v)$ and $\rho(\gamma, v) \rightarrow$ $F(\gamma) \gamma v^{\gamma-1}$. However, the explicit IR regulator remains mandatory for $\gamma<0$.

As a closing cross check, let us verify that for $\gamma<0$ and regularized

$$
\rho(-\beta, v)=F(-\beta)\left[\delta(v) \varepsilon^{-\beta}-\theta(v>\varepsilon) \beta v^{-1-\beta}\right],
$$

the basic convolution rule of Eq. (27) still holds: ${ }^{16}$

$$
\int d v_{1} d v_{2} \delta\left(v-v_{1}-v_{2}\right) \rho\left(-\beta, v_{1}\right) \rho\left(\gamma, v_{2}\right)=\rho(\gamma-\beta, v) .
$$

In terms of the Markovian process, the function $\rho(\gamma, v)$ for $\gamma>0$ represents adding more (soft) photons. The other $\rho(-\beta, v)$ function is undoing that (backward evolution).

In the context of the explanation of the physics of IFI in Sec. II, the presence of $\rho(\gamma, v)$ with negative $\gamma$ in the forward hemisphere in Eq. (25) is now perfectly

\footnotetext{
${ }^{16}$ In the $\varepsilon \rightarrow 0$ limit, of course.
}

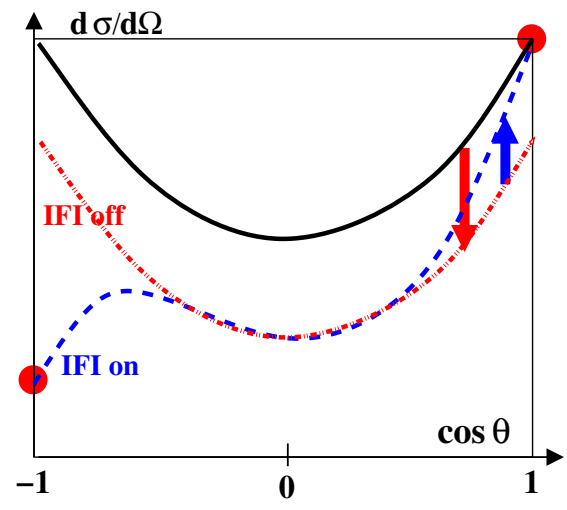

FIG. 7. The role of IFI. In forward scattering, the upward arrow (IFI) partially counteracts the action of the downward arrow (ISR + FSR).

understandable: $\rho\left(\gamma_{X}, r\right) \rho\left(\gamma_{X}, r^{\prime}\right)$ is undoing part of the ISR and FSR photon emission coming from $\rho\left(\gamma_{I}, v\right) \rho\left(\gamma_{F}, u\right)$ ! See the upward (blue) arrow in Fig. 7 for the corresponding graphical illustration.

In the numerical $\mathrm{MC}$ integration, it is not difficult to introduce a small IR regulator $\varepsilon$ into $\rho\left(\gamma_{X}, r\right)$ when $\gamma_{X}<0$. In the integrand for FOAM, this is done as a part of the mapping of the integration variables $r$ and $r^{\prime}$ into internal variables of FOAM.

Another issue is that the integrand becomes negative for $\gamma_{X}<0$, for $r>\varepsilon$, or for $r^{\prime}>\varepsilon$. This is handled in a standard way using weighted MC events with a nonpositive weight. In the actual integration by means of FOAM, the modulus of the integrand is used during the exploration stage, while in the following MC calculation of the integral, the MC events are weighted with the true signed distribution. The distribution of the $\mathrm{MC}$ weights in the second stage has two peaks: ${ }^{17}$ the bigger one close to +1 and smaller one near -1 ; see Fig. 8. More details on the mappings used in the construction of the integrand for FOAM in KKFoam program are given in Appendix B.

\section{NUMERICAL RESULTS FROM KKFOam AND KKMC}

In this section, we present results from the updated $\mathrm{v} 4.22$ of $\mathrm{KKMC}$, also referred to as KKMCee, the non-MC integrator KKsem, ${ }^{18}$ and the newly developed MC integrator (simulator) program KKFoam, based on the $\mathrm{C}++$ version of FOAM [30]. KKFOam implements the 5-dimensional integral of Eq. (29) including IFI, together with its 3-dimensional variant without IFI of Eq. (32). They will

\footnotetext{
${ }^{17}$ This entails a certain loss of integration precision, but it turns out to be affordable.

${ }^{18} \mathrm{KKsem}$ uses Gauss quadrature programs to integrate analytic formulas up to 3 dimensions. It was developed at the time of preparing Ref. [12].
} 


\section{Weight distribution Foam}

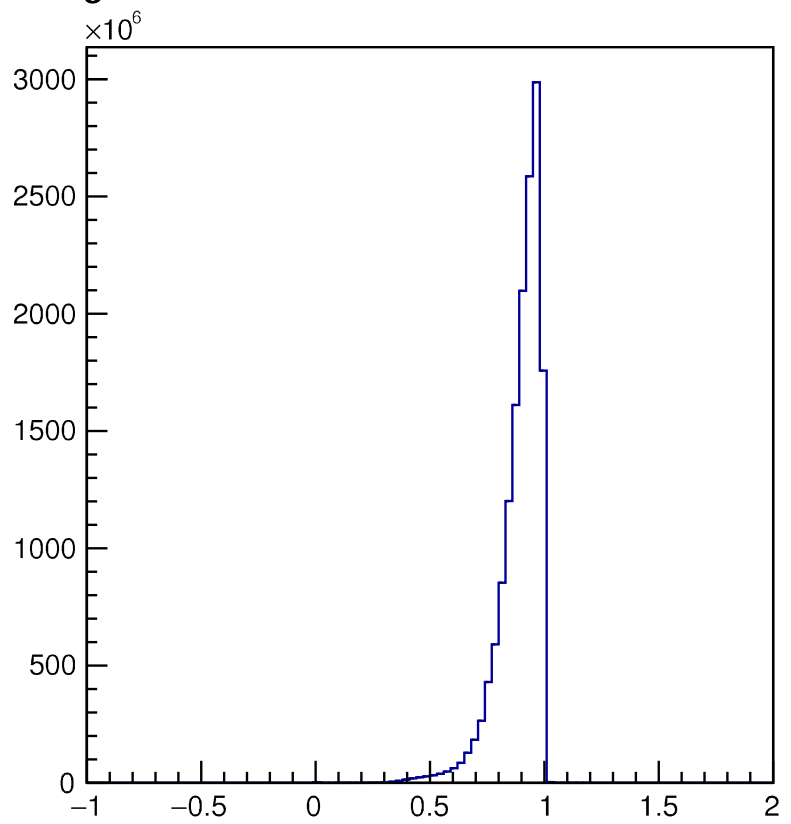

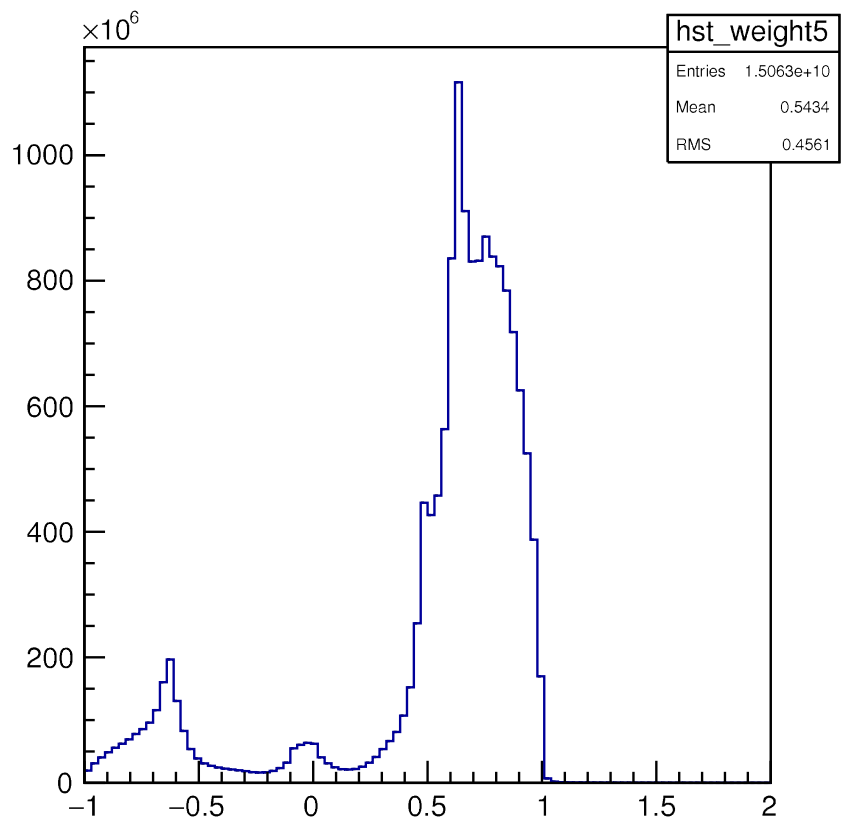

FIG. 8. The right-hand plot is an example of the MC weight distribution for calculating the total cross section using FOAM according to Eq. (29). The left-hand plot presents the MC weight distribution without IFI, see Eq. (32).

be often nicknamed in the following as KKFoam5 and KKFoam3, correspondingly.

Another subgenerator in KKFoam taking care of 2dimensional integration over $v$ and $\cos \theta$ will be used for reproducing and/or implementing old pure $\mathcal{O}\left(\alpha^{1}\right)$ results without resummation.

In KKFoam5 and KKFoam3, one may choose ISR and FSR structure functions with soft photon exponentiation and QED corrections up to $\mathcal{O}\left(\alpha^{0}\right), \mathcal{O}\left(\alpha^{1}\right)$ and $\mathcal{O}\left(\alpha^{2}\right)$, as defined in Tables I and II in Ref. [12]. Pure QED nonlogarithmic $\mathcal{O}\left(\alpha^{2}\right)$ corrections are $<10^{-5}$, hence are neglected for ISR, FSR and IFI. They should be included and evaluated more precisely in the future.

The Born cross section in both KKFoam5 and KKFoam3 is implemented using two types of subprograms of KKMC, both of them using spin amplitudes: either calculated in the global positioning of spin (GPS) scheme ${ }^{19}$ of Ref. [12] and labeled with GPS or CEEX, or using spin amplitudes of KORALZ [17] and labeled as EEX. Note that it is not possible to use EEX Born for IFI implementation; hence in KKFoam5 only GPS/CEEX Born amplitudes are implemented.

Electroweak and QCD corrections are included in KKFOam in the rescaled coupling constants of Born amplitudes, both for CEEX/GPS and EEX type, the same way as in KKMC. Contributions from nonfactorizable $\gamma \gamma$ and $\gamma Z$ boxes are also included; see also Eq. (33) for the details. Both KKMC and KKFoam use DIZET library of the $\mathcal{O}\left(\alpha^{1}\right)$ EW corrections [28] [including some of $\mathcal{O}\left(\alpha^{2}\right)$ ]

\footnotetext{
${ }^{19}$ This is a variant of Kleiss-Stirling method of Ref. [34].
}

and the method in which EW corrections are inserted into Born-like parts of the spin amplitudes in KKMC is essentially the same as in ZFITTER [13]. It is described in Eqs. (21-25) of Ref. [22]. This method protects completeness of the $\mathcal{O}\left(\alpha^{1}\right)$ content of the EW corrections. If there is any bias introduced in this method, then it has to be of $\mathcal{O}\left(\alpha^{2}\right)$.

It should be kept in mind that in KKFoam5, hard photon corrections are included in integrated form in the structure functions up to $\mathcal{O}\left(\alpha^{2}\right)$ for ISR and FSR, while for IFI they are not included - only the finite parts of the virtual $\mathcal{O}\left(\alpha^{1}\right)$ IFI corrections $(\gamma-Z$ boxes) are included there. (In KKFoam3, IFI is completely absent.)

The immediate short-term aim in this section is to prove that these programs correctly calculate $\sigma\left(v<v_{\max }\right)$ and $A_{\mathrm{FB}}\left(v_{\max }\right)$ with physical and technical precision $\delta A_{\mathrm{FB}} \sim$ $10^{-4}$ and $\delta \sigma / \sigma \sim 3 \times 10^{-4}$. This is a factor of 10 better than at LEP, but still a factor of 10 short of what needed for FCCee near the $Z$ resonance. An additional cutoff $|\cos \theta|<$ $c_{\max }$ will sometimes be imposed. An analysis for more realistic cuts will be presented in a separate publication. The IFI effect in $A_{\mathrm{FB}}$ depends strongly on the cutoff on the total photon energy $v_{\max }$, which will typically be varied between $v_{\max }=0.002$ and $v_{\max }=0.200$. As already pointed out in the Introduction, such a cutoff stronger that in typical LEP data analysis may be necessary at FCCee for the sake of better control of backgrounds and higher order QED effects. Moreover, the expectation is that semisoft photon resummation employed in KKFoam5 (taking into account the energy shift due to ISR in the $Z$ 
TABLE I. Table of various types of QED matrix elements, resummation methodology and phase space integration methods in the following numerical studies. "Semisoft" indicates that exact multiphoton M.E. with narrow resonance effects included. "Soft.+Col." indicates the use of collinear photon distribution functions (PDFs) for ISR and FSR. "GPS Born" means the use of Born spin amplitudes as in CEEX, while "EEX Born" indicates the use of Born of the EEX scheme. EW corrections are placed in the Born-like part of the spin amplitudes.

\begin{tabular}{|c|c|c|c|c|c|c|}
\hline MC Prog. & M.E. & Resum. & ISR & FSR & IFI & EW \\
\hline KKMC & CEEX2 & Semisoft & $\mathcal{O}\left(\alpha^{2}\right)$ & $\mathcal{O}\left(\alpha^{2}\right)$ & $\mathcal{O}\left(\alpha^{1}\right)$ & Yes \\
\hline KKMC & CEEX1 & Semisoft & $\mathcal{O}\left(\alpha^{1}\right)$ & $\mathcal{O}\left(\alpha^{1}\right)$ & $\mathcal{O}\left(\alpha^{1}\right)$ & Yes \\
\hline $\mathrm{KKMC}$ & CEEX0 & Semisoft & $\mathcal{O}\left(\alpha^{0}\right)$ & $\mathcal{O}\left(\alpha^{0}\right)$ & $\mathcal{O}\left(\alpha^{0}\right)$ & Yes \\
\hline $\mathrm{KKMC}$ & EEX3 & Soft.+Col. & $\mathcal{O}\left(\alpha^{3}\right)$ & $\mathcal{O}\left(\alpha^{2}\right)$ & None & Yes \\
\hline $\mathrm{KKMC}$ & EEX2 & Soft.+Col. & $\mathcal{O}\left(\alpha^{2}\right)$ & $\mathcal{O}\left(\alpha^{2}\right)$ & None & Yes \\
\hline $\mathrm{KKMC}$ & EEX1 & Soft.+Col. & $\mathcal{O}\left(\alpha^{1}\right)$ & $\mathcal{O}\left(\alpha^{1}\right)$ & None & Yes \\
\hline $\mathrm{KKMC}$ & EEX0 & Soft.+Col. & $\mathcal{O}\left(\alpha^{0}\right)$ & $\mathcal{O}\left(\alpha^{0}\right)$ & None & Yes \\
\hline KKsem2 & EEX Born & Soft.+Col. & $\mathcal{O}\left(\alpha^{2}\right)$ & $\mathcal{O}\left(\alpha^{2}\right)$ & None & Yes \\
\hline KKsem0 & EEX Born & Soft.+Col. & $\mathcal{O}\left(\alpha^{0}\right)$ & $\mathcal{O}\left(\alpha^{0}\right)$ & None & Yes \\
\hline KKFoam5 & GPS Born & Soft.+Col. & $\mathcal{O}\left(\alpha^{2}\right)$ & $\mathcal{O}\left(\alpha^{2}\right)$ & $\mathcal{O}\left(\alpha^{1}\right)$ & Yes \\
\hline KKFoam3 & EEX Born & Semisoft & $\mathcal{O}\left(\alpha^{2}\right)$ & $\mathcal{O}\left(\alpha^{2}\right)$ & None & Yes \\
\hline KKFoam2 & EEX Born & None & $\mathcal{O}\left(\alpha^{1}\right)$ & $\mathcal{O}\left(\alpha^{1}\right)$ & $\mathcal{O}\left(\alpha^{1}\right)$ & Yes \\
\hline
\end{tabular}

propagator) will work fairly well in this cutoff range near the $Z$ pole. $^{20}$

In the following analysis, event selection will be examined in terms of two variables only, $\cos \theta$ for the angle between $e^{-}$and $\mu^{-}$and $v=1-M_{\mu \mu}^{2} / s$. The variable $v$ represents approximately the total energy of all ISR and FSR photons, in units of the beam energy. (More results for realistic selection cuts will be shown in the next paper.) Of course, once harder photons are allowed, the definition of $\cos \theta$ is no longer unique. For the KKMC results, we will use the $\cos \theta$ definition of Ref. [8] unless otherwise stated-see the following Sec. IV B for more discussion of other choices of $\cos \theta$ and their precise definitions.

\section{A. Outline of the numerical investigations}

We have conducted numerical studies with three different programs, KKMC, KKsem and KKFoam, featuring several variants of QED matrix elements and different types of phase space integration. For the convenience of the reader, we summarize in Table I all types of programs and QED matrix elements (M.E.) used in them. The CEEX matrix element of $\mathrm{KKMC}$ for IFI component is rated in the table as $\mathcal{O}\left(\alpha^{1}\right)$ because of missing nonsoft $\mathcal{O}\left(\alpha^{2}\right)$ parts of the pentabox diagrams specified in Fig. 5 in Ref. [12], but the soft/infrared parts of these diagrams are included thanks to the semisoft resummation technique. It would be desirable to include these pentaboxes in a future version of KKMC. ${ }^{21}$

Let us outline the plan of the following tests which will lead to new estimates of the theoretical uncertainty of the IFI calculation:

\footnotetext{
${ }^{20}$ On the other hand, strict YFS soft photon approximation neglecting the ISR energy shift in the $Z$ propagator is expected to be adequate for our precision requirements only for $v_{\max } \leq 10^{-4}$.

${ }^{21}$ Another urgent desirable update of the M.E. in KKMC would be inclusion of the $\alpha^{3} \ln ^{3}\left(s / m_{e}^{2}\right)$ corrections.
}

(i) In the following Sec. IV B, we shall find that the influence of the choice of the muon scattering angle on the measurement of $A_{\mathrm{FB}}$ is negligible.

(ii) Section IV C is devoted to a calibration exercise in which the correctness of the MC integration is checked by comparing the cutoff dependence of $\sigma\left(v_{\max }\right)$ and $A_{\mathrm{FB}}\left(v_{\max }\right)$ from three programs, KKMC, KKsem and KKFoam, with IFI switched off. It is done first for a maximally simple variant of the QED matrix element with resummation and then for the best one.

(iii) In the next step, in Sec. IV D, the IFI effect in $A_{\mathrm{FB}}$ is examined in the results for $A_{\mathrm{FB}}\left(v_{\max }\right)$ from KKMC and KKFoam for a maximally simple and the best QED matrix element separately for three energies $s^{1 / 2}=10,87.9,94.3 \mathrm{GeV}$.

(iv) Section IVE is devoted to the difference $\Delta A_{\mathrm{FB}}^{\mathrm{IFI}}\left(v_{\max }\right)=A_{\mathrm{FB}}^{\mathrm{IFI}}\left(v_{\max }, s_{+}\right)-A_{\mathrm{FB}}^{\mathrm{IFI}}\left(v_{\max }, s_{-}\right), \quad$ in which the IFI effect cancels. Results from KKMC and KKFOam for this difference will be compared. $\Delta A_{\mathrm{FB}}$ is directly related to the measurement of $\alpha_{\mathrm{QED}}\left(M_{Z}\right)$ at FCCee.

(v) Finally, in Sec. IV F results for the energy difference $\Delta A_{\mathrm{FB}}^{\mathrm{IFI}}\left(v_{\max }\right)$ from KKMC will be analyzed for QED matrix elements with an increasing level of sophistication in order to estimate its theoretical uncertainty due to missing higher orders of QED.

\section{B. On the choice of the scattering angle $\theta$}

In the limit when all photons are very soft, the momenta of the final muons are back to back and the scattering angle $\theta$ between $e^{-}$and $\mu^{-}$is unique. Once at least one photon becomes energetic, the final muons are not back to back and there are many possible definitions of the effective $\theta$. Using $\theta^{(1)}=\angle\left(e^{-}, \mu^{-}\right)$or $\theta^{(2)}=\angle\left(e^{+}, \mu^{+}\right)$is not a favorable choice experimentally, because it does not exploit 
fully the power of the tracker detector, which detects both $\mu^{ \pm}$equally well.

An example of a choice favorable for experiments, taking full advantage of the very good angular resolution of the muon detectors (trackers), which is much higher than the energy resolution, is that of ref [33]:

$$
\begin{aligned}
\cos \theta^{*} & =y_{1} \cos \theta_{1}-y_{2} \cos \theta_{2}, \\
y_{1} & =\frac{\sin \theta_{2}}{\sin \theta_{1}+\sin \theta_{2}}, \quad y_{2}=\frac{\sin \theta_{1}}{\sin \theta_{1}+\sin \theta_{2}}, \\
& y_{1}+y_{2}=1 .
\end{aligned}
$$

However, it was shown in Ref. [8] that analytic evaluation of the IFI effect according to the $\mathcal{O}\left(\alpha^{1}\right)$ QED matrix element can be easily done using

$$
\begin{array}{rlr}
\cos \theta^{\star} & =x_{1} \cos \theta^{(1)}+x_{2} \cos \theta^{(2)}, & x_{i}=q_{i}^{0} /\left(q_{1}^{0}+q_{2}^{0}\right), \\
x_{1}+x_{2} & =1 .
\end{array}
$$

We use this choice for most of the numerical results presented in this work, unless otherwise stated. Moreover, in Ref. [8] compact analytic results were obtained for a charge asymmetry defined using the first moment,

$$
\tilde{A}_{\mathrm{FB}}^{*}=\frac{3}{2} \int_{-1}^{1} \cos \theta^{*} \frac{d \sigma}{\sigma},
$$

instead of the conventional forward-backward asymmetry $A_{\mathrm{FB}}=\left(\sigma_{F}-\sigma_{B}\right) / \sigma$.

For KKFOam, the choice of $\cos \theta$ is irrelevant as long as all photons are sufficiently ${ }^{22}$ soft. Once at least one photon becomes energetic, the $\mathcal{O}\left(\alpha^{1}\right)$ contribution calculated for a well-defined choice of $\cos \theta$ should be included in KKFoam. So far, this is not yet done-it should be done in the next version. Most likely, the preferred choice for $\mathrm{KKFOam}$ will be $\cos \theta^{\star}$.

On the other hand, $\mathrm{KKMC}$ is a regular MC event generator providing four-momenta of both muons (and all photons); hence it provides a prediction for $A_{\mathrm{FB}}$ with any definition of $\cos \theta$. Let us examine, using KKMC, how different the QED predictions for $A_{\mathrm{FB}}$ are for the above two choices of $\theta$ when $v<0.2$. Figure 9 shows that the difference between $A_{\mathrm{FB}}^{*}$ and $A_{\mathrm{FB}}^{*}$ is below expected FCCee experimental precision of $\delta A_{\mathrm{FB}} \sim 3 \times 10^{-5}$, i.e. all of our analysis for $\cos \theta^{*}$ is valid for $\cos \theta^{\star}$ and vice versa.

Using KKMC, it is easy to examine the difference between $\tilde{A}_{\mathrm{FB}}^{*}$ and $A_{\mathrm{FB}}^{*}$. Figure 10 shows that such a difference might be sizable, up to $\sim 1 \%$. However, the difference in the IFI component could cancel between two calculations-for instance, we have checked that it does cancel in the difference between KKMC and KKsem, for IFI switched on.

\footnotetext{
${ }^{22}$ Sufficiently from the point of view of the FCCee precision.
}

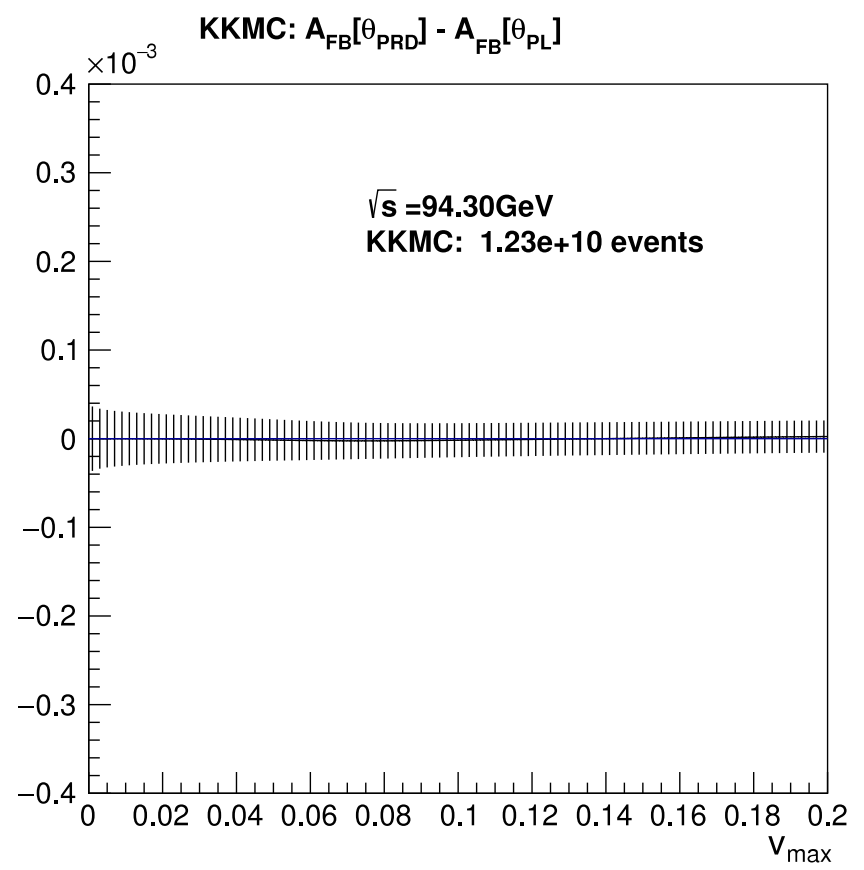

FIG. 9. The difference between $A_{\mathrm{FB}}^{\star}$ and $A_{\mathrm{FB}}^{\bullet}$, from KKMC at $10 \mathrm{GeV}$ with IFI on.

\section{Baseline calibration, ISR + FSR without IFI}

Let us start with the baseline calibration of the MC tools at the precision level $\sim 10^{-5}$ at $\sqrt{s_{-}}=87.9 \mathrm{GeV}$ and $\sqrt{s_{+}}=94.3 \mathrm{GeV}$. Although KKsem does not include IFI, it is still useful for checking the normalization of both KKMC and KKFoam. Of course, normalization is irrelevant for our main observable, $A_{\mathrm{FB}}\left(v_{\max }\right)$, but it is still profitable

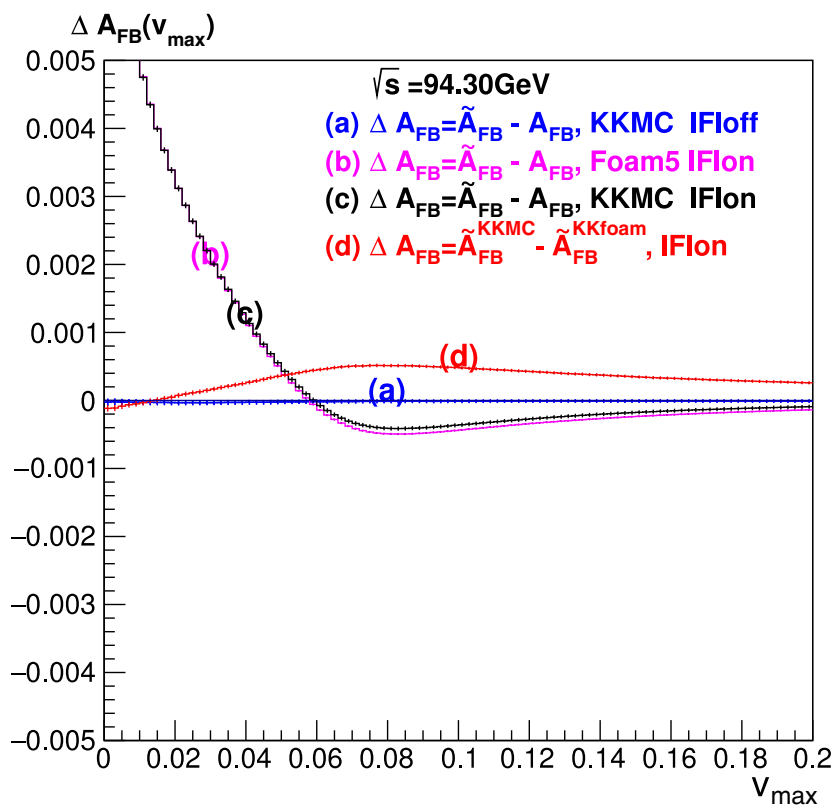

FIG. 10. The difference between $A_{\mathrm{FB}}$ and $\tilde{A}_{\mathrm{FB}}$ at $94.3 \mathrm{GeV}$ with IFI on. 
to test it, simply because some technical problem that would be evident in $\sigma\left(v_{\max }\right)$ could produce a small annoying effect in $A_{\mathrm{FB}}$ as well. Thus, it is better to keep an eye on both of these.

As already underlined, our main aim in the present study is a precise prediction for $A_{\mathrm{FB}}$ at two energies $\sqrt{s_{ \pm}}=87.9$ near $Z$ resonance. However, in order to get better confidence in the implementation of the QED matrix element, we will also check $A_{\mathrm{FB}}$ at $\sqrt{s}=10 \mathrm{GeV}$, where the $Z$ resonance is negligible, and at $\sqrt{s}=M_{Z}$, where the suppression of IFI due to the long life time of the $Z$ is maximal.

Let us start with a purely technical test with IFI off at $\sqrt{s}=94.3,87.9$ and $10 \mathrm{GeV}$, presented in Fig. 11. In the LHS plot, all cross sections $\sigma\left(v_{\max }\right)$ are divided by the reference cross section from KKsem. All calculations are at simplistic exponentiated $\mathcal{O}\left(\alpha^{0}\right)$ QED including ISR and FSR, but without IFI. Different types of the Born matrix element, EEX or GPS, are used. Very good agreement is seen, up to statistical error $\delta \sigma / \sigma \sim 3 \times 10^{-5}$. The agreement for $A_{\mathrm{FB}}$ is also very good, essentially up to statistical error $\delta A_{\mathrm{FB}} \sim 1 \times 10^{-5}$ at $\sqrt{s_{ \pm}}$. The above equality of the KKFOam and KKsem results is very important, because it illustrates/proves the quality of the MC integrators-it should be kept in mind that for IFI off they integrate exactly the same 3-dimensional integrand. Even more significant is the agreement, to within $\delta \sigma / \sigma \sim 1 \times 10^{-5}$ near the $Z$ resonance, of KKMC with the other two programs for the simplified EEX0 matrix element. This is because for MC statistics of $2 \times 10^{10}$ events one may expect problems with rounding errors in the accumulation of the weights in the histograms. ${ }^{23}$ The slightly bigger discrepancy beyond statistical error of $\delta A_{\mathrm{FB}} \sim 3 \times 10^{-5}$ for $10 \mathrm{GeV}$ is not yet statistically significant and not so important for our aims.

We conclude that the technical precision of the MC numerical integration in all three programs, KKsem, KKFOam and KKsem, is satisfactory for our needs. Moreover, the above test is also important due to the fact that the IFI effect is added in KKMC by reweighting MC events generated without IFI. Hence, the technical precision established for the non-IFI mode persists when IFI is switched on.

In Fig. 12, we continue baseline testing without IFI, now with $\mathcal{O}\left(\alpha^{2}\right)$ exponentiated ISR and FSR. The relative differences $\delta \sigma / \sigma$ between KKMC and KKFoam vs KKsem are examined. It is done for the CEEX/GPS and EEX Born matrix element. The relative difference $\delta \sigma / \sigma \sim 3 \times 10^{-4}$ for KKMC confirms all older tests in Ref. [12], rated at the $\sim 1 \times$ $10^{-3}$ level. $^{24}$ On the other hand, the differences in $A_{\mathrm{FB}}$ between KKMC and KKFoam or KKsem are again of the

\footnotetext{
${ }^{23}$ Running in parallel on 100 nodes and combining the histograms afterwards helps to reduce this problem.

${ }^{24}$ This is not a high priority, but we shall try later to find the source of these differences in the normalization.
}

order of the statistical error, which is $\sim 3 \times 10^{-5}$, except $\sqrt{s}=10 \mathrm{GeV}$, where it is slightly bigger.

The main result of the tests presented in Figs. 11 and 12 is that the basic technical precision (in the MC integration) of KKMC and KKFOam3 near the $Z$ resonance is generally better than $\delta A_{\mathrm{FB}} \sim 3 \times 10^{-5}$. The implementation of QED photonic corrections for ISR and FSR (no IFI) up to $\mathcal{O}\left(\alpha^{2}\right)$ was also tested at this precision level. ${ }^{25}$

\section{IFI contribution to $A_{\mathrm{FB}}$ from KKMC and KKFoam}

Let us now increase the level of sophistication by one important step-including IFI. This will be done first in the simpler case (A) for ISR, FSR and IFI at the level $\mathcal{O}_{\exp }\left(\alpha^{0}\right)$ with exponentiation, and next in the case (B) for exponentiated IFI at the level $\mathcal{O}_{\exp }\left(\alpha^{1}\right)$, accompanied by ISR and FSR up to exponentiated $\mathcal{O}_{\exp }\left(\alpha^{2}\right)$.

In case (A), results for $A_{\mathrm{FB}}\left(v_{\max }\right)$ from KKMC and KKFOam are shown in Fig. 13, while in case (B), the results are shown in Fig. 14, for energies $\sqrt{s}=87.9,94.3$, $10 \mathrm{GeV}$ in both cases. The absolute predictions for $A_{\mathrm{FB}}$ from KKMC and KKFOam are seen in the LHS plots of the these figures. The differences in $A_{\mathrm{FB}}$ due to switching on the IFI contribution are quite sizable and rising quickly for $v_{\max } \leq 0.06$, up to $5 \%$ for $v_{\max } \leq 0.002$.

The IFI contribution to $A_{\mathrm{FB}}$ is shown more clearly in the RHS plots of Figs. 13 and 14, where the absolute predictions for the IFI effect in $A_{\mathrm{FB}}$ from $\mathrm{KKMC}$ and KKFOam are presented. The most important result is the one represented by the red curve (c) in the RHS figures in Fig. 14. It represents the difference between KKMC and KKFOam for the IFI contribution. This crucial difference is up to $\delta A_{\mathrm{FB}} \sim 5 \times 10^{-4}$. (It will be analyzed carefully one more time in the next section.) It is definitely above the technical precision level $\delta A_{\mathrm{FB}}<3 \times 10^{-5}$, established previously in case of IFI switched off.

How can we understand the above result? In the case of Fig. 13 where both KKMC and KKFoam are at the same $\mathcal{O}_{\text {exp }}\left(\alpha^{0}\right)$ level for ISR, FSR and IFI, with semisoft resummation of IFI, the source of the difference is a different treatment of the matrix element far away from the infrared point $v_{\max }=0$. Remembering that the energy shift in the $Z$-resonance propagator is properly taken into account in the semisoft approximation, the difference between KKMC and KKFoam should be proportional to $v_{\max }$ and should vanish for $v_{\max } \rightarrow 0$. This is what we see in Fig. $13 .^{26}$

\footnotetext{
${ }^{25}$ This is not true for KKFOam, where subprograms with and without IFI are independent modules generating their own different MC events.

${ }^{26}$ The slight difference at $v_{\max } \rightarrow 0$ for $\sqrt{s}=10 \mathrm{GeV}$ can be traced to small spikes in the $0.99<|\cos \theta|<1$ range, to be examined separately. It goes away for realistic experimental cutoffs.
} 

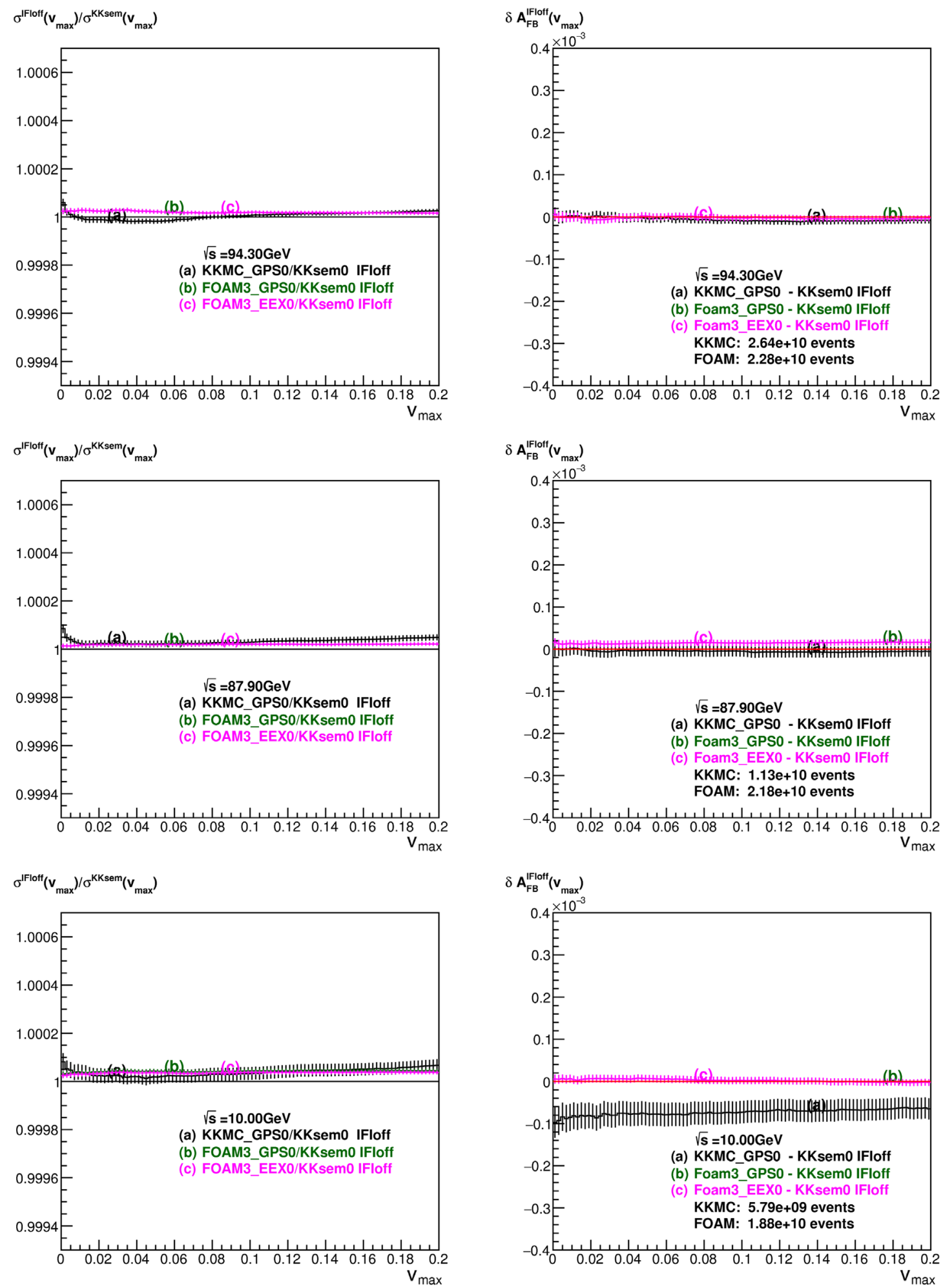

FIG. 11. Technical test, $\mathcal{O}_{\exp }\left(\alpha^{0}\right)$ ISR + FSR without IFI at $94.3,87.9$ and $10 \mathrm{GeV}$.

In the case of Fig. 14, the difference between KKMC and KKFoam should reflect the fact that in KKMC the entire $\mathcal{O}_{\exp }\left(\alpha^{1}\right)$ real and virtual contributions are included, while in KKFoam5, the $\mathcal{O}_{\text {exp }}\left(\alpha^{1}\right)$ real contribution is incomplete.
This could increase the difference between KKMC and KKFOam. In fact it changes sign and increases the difference by at most a factor of 2. This can be seen as unexpected. In order to have an idea how big the 

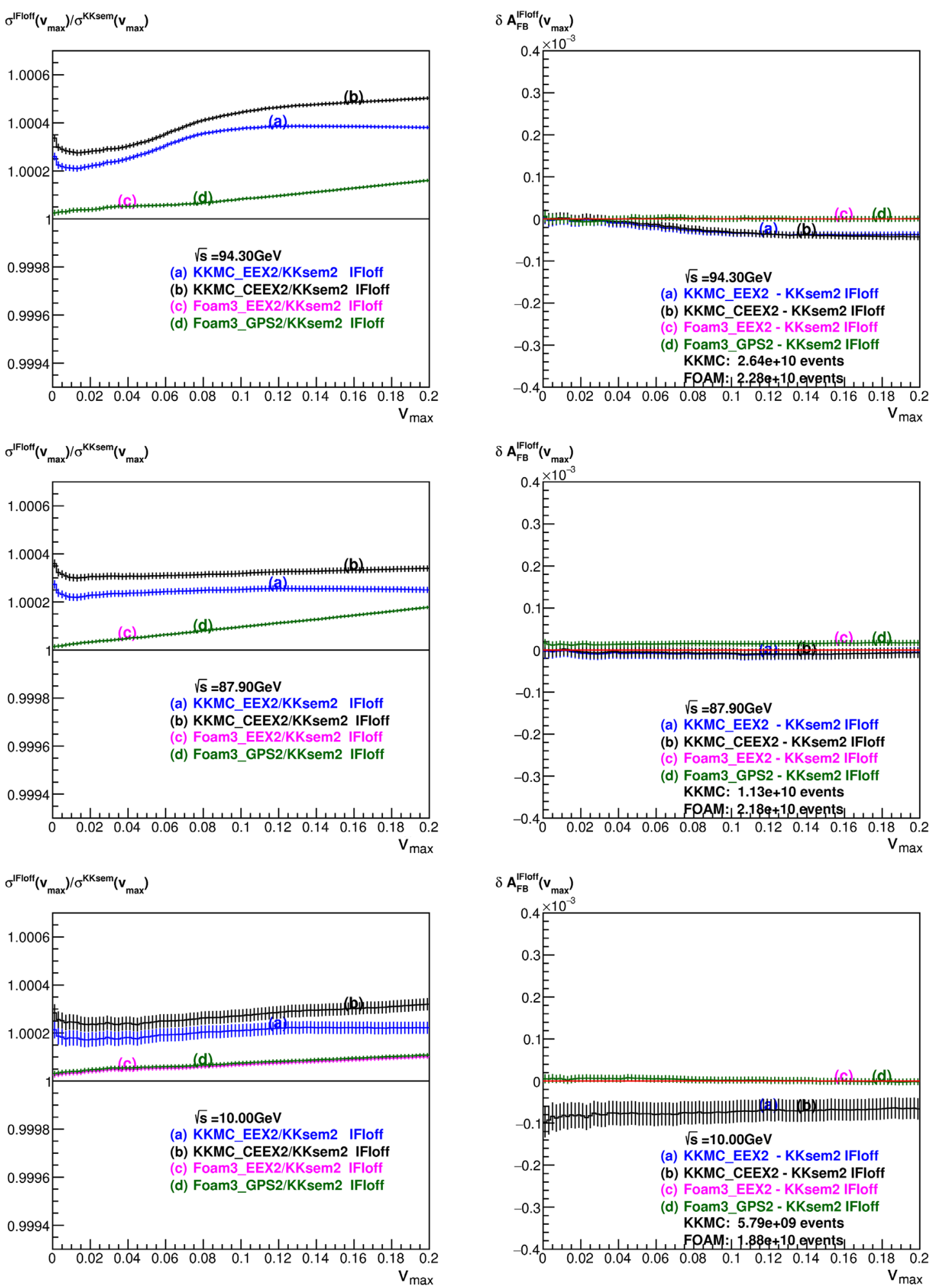

FIG. 12. Results with $\mathcal{O}_{\exp }\left(\alpha^{2}\right)$ ISR + FSR without IFI at $94.3,87.9$ an $10 \mathrm{GeV}$.

$\mathcal{O}_{\text {exp }}\left(\alpha^{1}\right)$ real photon IFI contribution can be, we have also included this contribution [curve (e)] in Fig. 14, subtracting the soft component in an ad hoc manner. As we see, curve (e) typically has the same sign as the difference between KKMC and KKFoam shown in curve (c), but is a factor of
3-4 bigger. Apparently, KKFoam includes most of the $\mathcal{O}\left(\alpha^{1}\right)$ hard photon IFI contribution. ${ }^{27}$

\footnotetext{
${ }^{27}$ It would be interesting to include this missing $\mathcal{O}_{\text {exp }}\left(\alpha^{1}\right)$ real photon IFI contribution in KKFOam.
} 

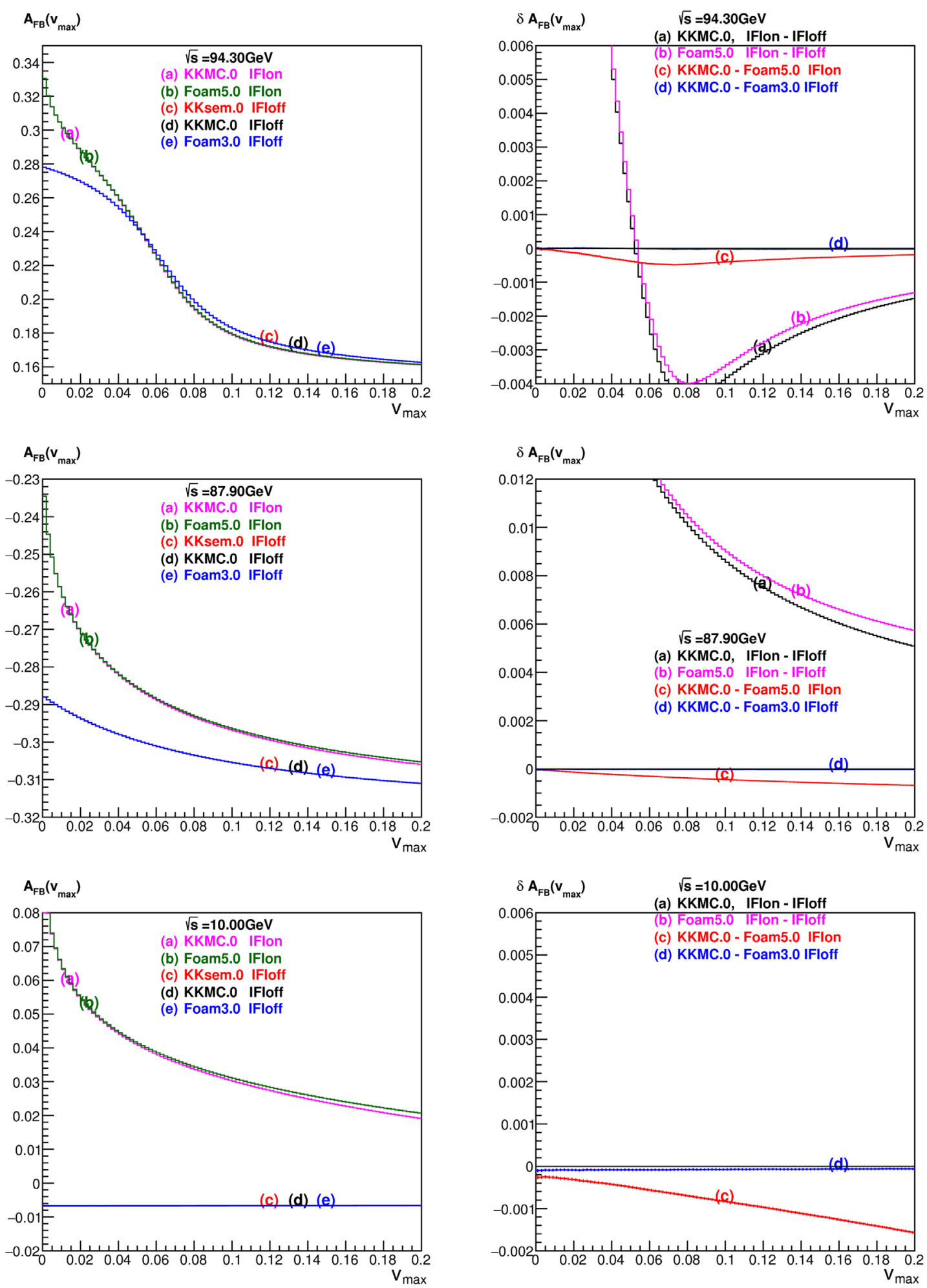

FIG. 13. Results with $\mathcal{O}_{\exp }\left(\alpha^{0}\right)$ ISR + FSR and IFI at $94.3 \mathrm{GeV}, 87.9 \mathrm{GeV}$ and $10 \mathrm{GeV}$.

The inclusion of QED $\mathcal{O}_{\exp }\left(\alpha^{1}\right)$ virtual corrections and box diagrams was done in KKFOam following the prescription of Eq. (33). The pure $\mathcal{O}\left(\alpha^{1}\right)$ numerical results in Fig. 14 were reproduced using analytic formulas of
Refs. [8,33], which are collected and tested numerically one more time in Appendix C.

In spite of the incompleteness of the $\mathcal{O}\left(\alpha^{1}\right)$ IFI in KKFoam, the above result makes us confident that we 

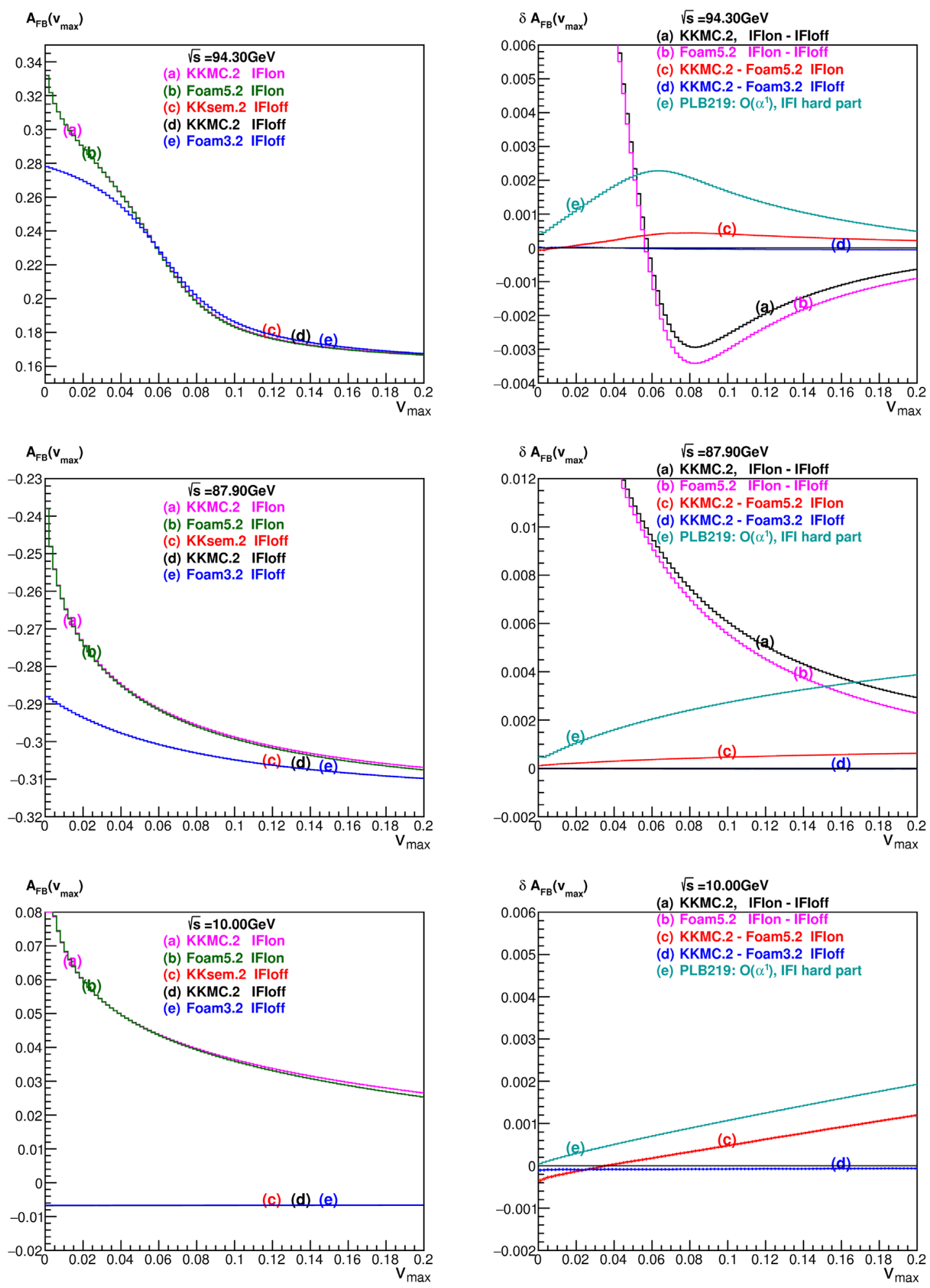

FIG. 14. Results with $\mathcal{O}_{\exp }\left(\alpha^{2}\right)$ ISR + FSR and $\mathcal{O}_{\exp }\left(\alpha^{1}\right)$ IFI at $94.3,87.9$ and $10 \mathrm{GeV}$.

are quite close to reaching our first intermediate goal of controlling the IFI effect in $A_{\mathrm{FB}}$ at the level of $\delta A_{\mathrm{FB}} \sim 10^{-4}$ in the semisoft resummation regime $\left(v_{\max } \leq 0.06\right)$.

Let us also finally show just one example of the entire angular distribution $d \sigma / d \cos \theta$ from KKFOam and KKMC, simply because agreement in $A_{\mathrm{FB}}$ does not necessarily imply agreement in the angular distributions. In Fig. 15, such a comparison is done for a relatively mild cutoff $v_{\max }=0.02$ on the total photon energy. The angular distributions agree to within $0.005 \%$ as expected. 

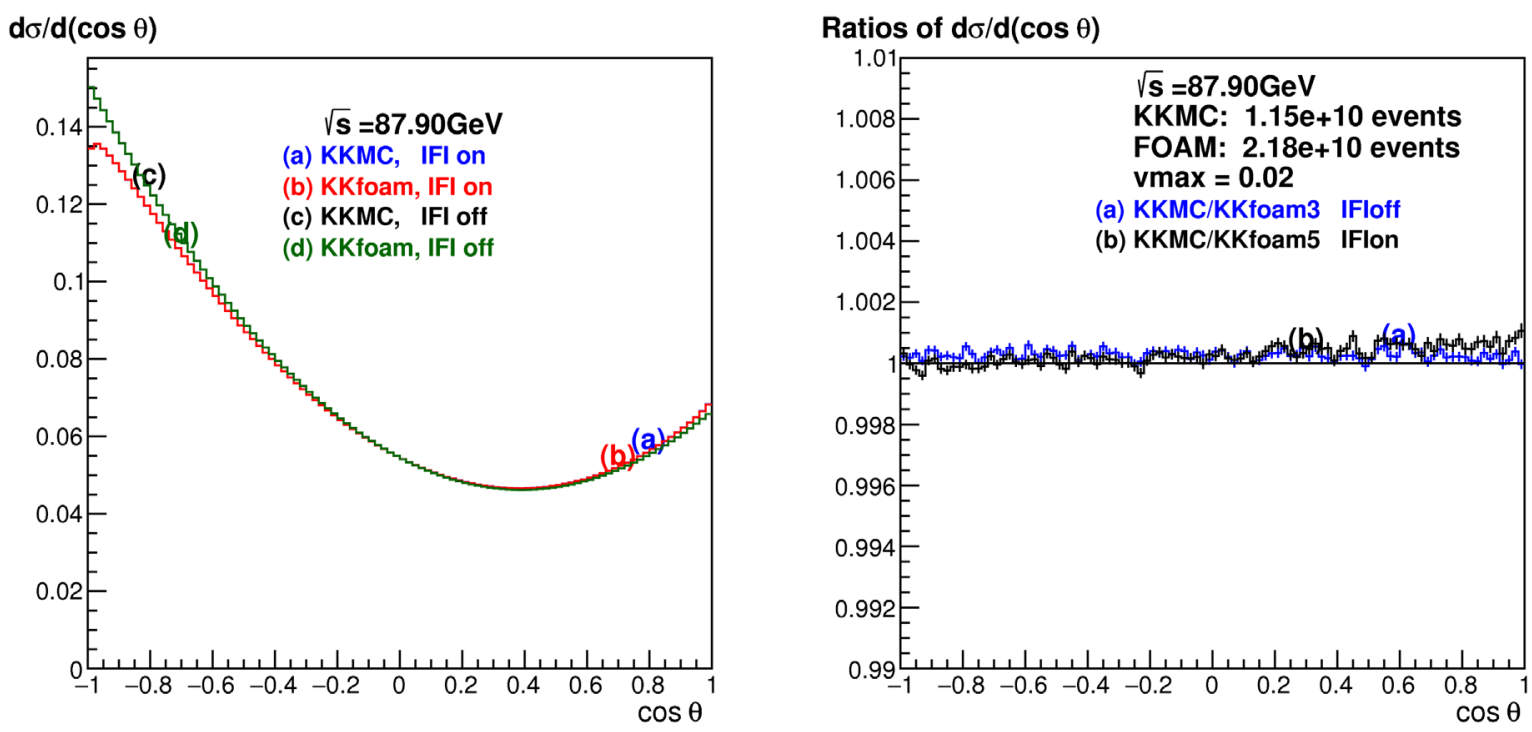

FIG. 15. Comparison between KKMC and KKFoam for the angular distribution for the cutoff on total photon energy $v_{\text {max }}=0.02$.

\section{E. $A_{\mathrm{FB}}\left(s_{ \pm}\right)$from KKMC and KKFoam in presence of IFI}

As explained in Ref. [5], the QED coupling constant $\alpha_{\mathrm{QED}}\left(M_{Z}\right)$ is closely related to $A_{\mathrm{FB}}\left(s_{ \pm}\right)$, but the exact relation is not straightforward and we are not trying to reproduce it. We limit our interest to the propagation of errors from $A_{\mathrm{FB}}\left(s_{ \pm}\right)$to $\alpha_{\mathrm{QED}}\left(M_{Z}\right)$, which is simpler and can be read from Eq. (4.9) in Ref. [5]. For our purpose, it will be enough to use a simplified version of this equation,

$$
\left.\frac{\delta \alpha_{\mathrm{QED}}}{\alpha_{\mathrm{QED}}}\right|_{M_{Z}} \simeq \frac{\delta A_{\mathrm{FB}}\left(s_{+}\right)-\delta A_{\mathrm{FB}}\left(s_{-}\right)}{A_{\mathrm{FB}}\left(s_{+}\right)-A_{\mathrm{FB}}\left(s_{-}\right)}
$$

which is valid for small $\delta A_{\mathrm{FB}}\left(s_{ \pm}\right)$and/or when there are no strong cancellations between them. ${ }^{28}$ This will be true in the following numerical examples, and we shall show typically the numerator $\delta A_{\mathrm{FB}}\left(s_{+}\right)-\delta A_{\mathrm{FB}}\left(s_{-}\right)$along with the uncertainties $\delta A_{\mathrm{FB}}\left(s_{ \pm}\right)$.

Having the above in mind we reexamine the comparisons between KKMC and KKFOam of the previous section for this $\Delta A_{\mathrm{FB}}$.

From now on, we impose a realistic cutoff $|\cos \theta|<0.9$ in the tests; however the cutoff has little influence on the resulting $A_{\mathrm{FB}}$. To start with, in Fig. 16 we show $A_{\mathrm{FB}}\left(v_{\max }\right)$ from KKMC at $\sqrt{s_{ \pm}}$with IFI switched on/off and with the best QED matrix element in KKMC. The $A_{\mathrm{FB}}$ changes sign between these two energies. On the other hand, IFI keeps the same sign, hence we expect partial cancellation of the IFI effect in the $\alpha_{\mathrm{QED}}\left(M_{Z}\right)$. We do not pursue the reconstruction of $\alpha_{\mathrm{QED}}\left(M_{Z}\right)$ and only plot the difference of IFI effect between two energies in the LHS of Fig. 16 as a guide.

\footnotetext{
${ }^{28}$ We thank P. Janot for pointing this out to us.
}

We have produced the same figure for KKFoam, but we do not show it here, because it looks essentially the same as Fig. 16. What is more interesting is to reexamine the difference between KKMC and KKFOam,

$$
\delta A_{\mathrm{FB}}\left(s_{ \pm}\right)=\left.A_{\mathrm{FB}}\left(s_{ \pm}\right)\right|_{\mathrm{KKMC}}-\left.A_{\mathrm{FB}}\left(s_{ \pm}\right)\right|_{\mathrm{KKFoam}},
$$

already shown in curve (c) of Fig. 14, and its difference between two energies $\sqrt{s_{ \pm}}$,

$$
\Delta \delta A_{\mathrm{FB}}=\delta A_{\mathrm{FB}}\left(s_{+}\right)-\delta A_{\mathrm{FB}}\left(s_{-}\right),
$$

relevant for the uncertainty in the measurement of $\alpha_{\mathrm{QED}}\left(M_{Z}\right)$. We are interested in the above quantity primarily for IFI switched on. This quantity is shown in Fig. 17; see curve (c) there. It turns out that $\Delta \delta A_{\mathrm{FB}} \leq$ $2 \times 10^{-4}$ within the interesting range of photon energy cutoff $v_{\max } \leq 0.1$. In Fig. 17, we have also included two dashed lines ${ }^{29}$ marking the band of the present uncertainty $\delta \alpha_{\mathrm{QED}} / \alpha_{\mathrm{QED}}\left(M_{Z}\right)=1.1 \times 10^{-4}$ according to Ref. [35]. The aim of FCCee is of course to get a substantially smaller error than that.

The main contribution to $\Delta \delta A_{\mathrm{FB}}$ in curve (c) comes from the uncertainty in the IFI implementation (most likely in KKFOam), as can be seen from curve (d) in Fig. 17, which represents $\Delta \delta A_{\mathrm{FB}}$ for IFI switched off. The aim of future work will be to get $\Delta \delta A_{\mathrm{FB}} \leq 3 \times 10^{-5}$ for IFI on, that is to the same level as for IFI off, in the semisoft regime $v_{\max } \leq 0.06$.

The above $\Delta \delta A_{\mathrm{FB}} \leq 2 \times 10^{-4}$ can be treated as an (over) conservative estimate of the uncertainty of the IFI prediction for KKMC in the semisoft regime, which is much

\footnotetext{
${ }^{29}$ The dashed lines of the band are at $\pm 1.1 \times 10^{-4} \mid A_{\mathrm{FB}}\left(s_{+}\right)-$ $\left.A_{\mathrm{FB}}\left(s_{+}\right)\right|_{v_{\max } \rightarrow 0}= \pm 0.57 \times 10^{-4}$.
} 
KKMC: $A_{F B}\left(v_{\max }\right), \mid \cos (\theta \mid<0.9$

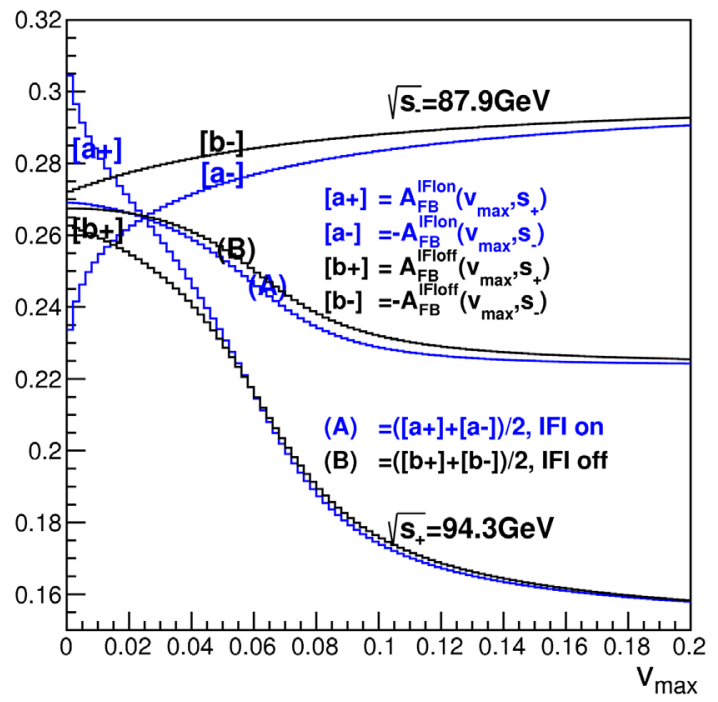

$A_{F B}^{|F|}\left(v_{\text {max }}\right)=A_{F B}^{I F \mid o n}\left(v_{\max }\right)-A_{F B}^{\mid F l o f f}\left(v_{\max }\right), \mid \cos (\theta \mid<0.9$

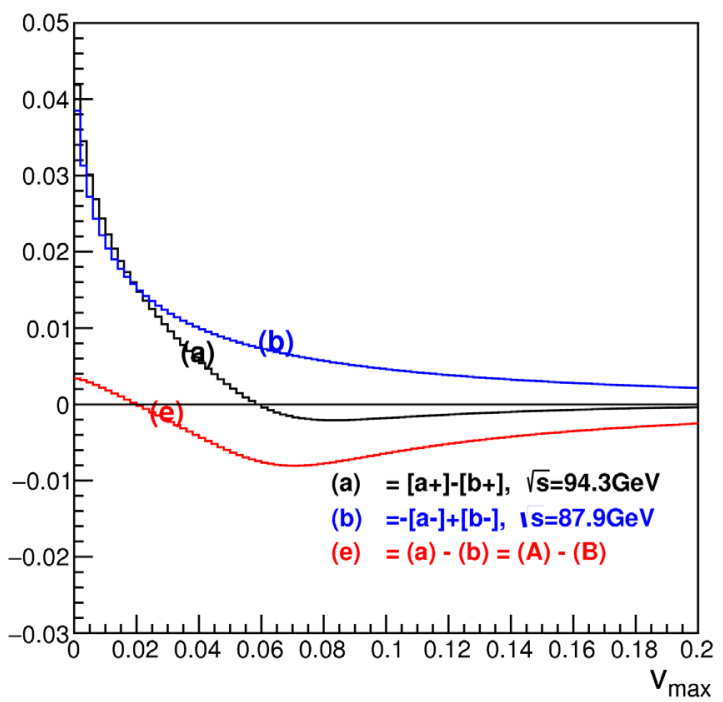

FIG. 16. $A_{\mathrm{FB}}\left(v_{\max }, s_{ \pm}\right)$from KKMC with $\mathcal{O}_{\exp }\left(\alpha^{2}\right)$ ISR + FSR and $\mathcal{O}_{\exp }\left(\alpha^{1}\right)$ IFI.

better than the LEP-era estimate but still not up to the needs of FCCee. A less conservative estimate will be provided in the next section.

\section{F. On $A_{\mathrm{FB}}$ for $\mathcal{O}_{\exp }\left(\alpha^{i}\right), i=0,1,2$ in $\mathrm{KKMC}$}

The differences between KKMC and KKFoam provide much valuable information, because the two programs

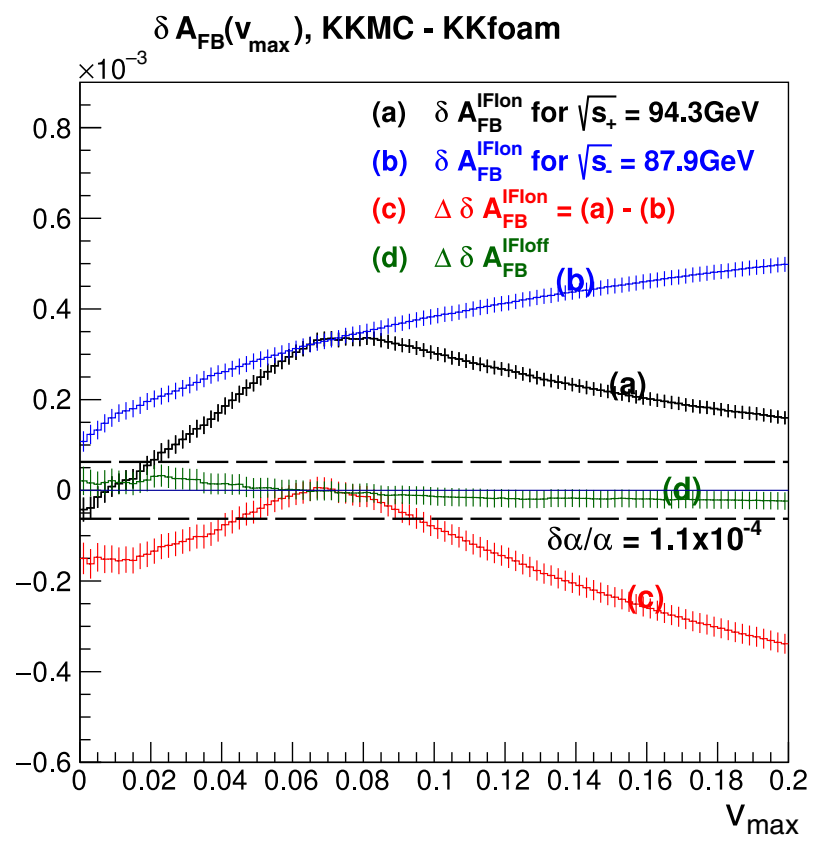

FIG. 17. Difference $\delta A_{\mathrm{FB}}\left(v_{\max }\right)$ between $A_{\mathrm{FB}}$ from KKMC and KKFOam and their difference between two energies $\sqrt{s_{ \pm}}$. IFI is switched on/off in both KKMC and KKFoam. The band marked with a dashed line corresponds to the precision estimate of the $\alpha_{\mathrm{QED}}\left(M_{Z}\right)$ of Ref. [35]. differ quite a lot technically (MC soft photon phase space integration vs analytic integration), while implementing the same physics of QED corrections. However, KKMC alone offers interesting insight into missing higher order QED corrections related to IFI. ${ }^{30}$

In KKMC, one may choose three types of the QED multiphoton matrix element with resummation at increasing sophistication levels, $\mathcal{O}_{\exp }\left(\alpha^{i}\right), i=0,1,2$. In Fig. 18 we examine differences in the IFI contribution $A_{\mathrm{FB}}^{\mathrm{IFI}}\left(v_{\max }\right)$ between $\mathcal{O}\left(\alpha^{2}\right)$ and $\mathcal{O}\left(\alpha^{1}\right)$ and also between $\mathcal{O}\left(\alpha^{1}\right)$ and $\mathcal{O}\left(\alpha^{0}\right)$. In all of them, IFI may be switched on or off. Complete non-IR $\mathcal{O}\left(\alpha^{1}\right)$ corrections are included in the $\mathcal{O}\left(\alpha^{i}\right), i=1,2$ case while in the $\mathcal{O}\left(\alpha^{0}\right)$ case, only the IR part of exponentiated IFI is implemented. In the most sophisticated case of the $\mathcal{O}_{\exp }\left(\alpha^{2}\right)$ QED matrix element in $\mathrm{KKMC}$, only pure nonlog photonic corrections are missing. ${ }^{31}$

In the LHS of Fig. 18, we show plots of the IFI component for all three cases $\mathcal{O}_{\exp }\left(\alpha^{i}\right), i=0,1,2$, while in the RHS we see the differences, for the two energy points $\sqrt{s_{ \pm}} \cdot 32$

The most important difference in Fig. 18, between $A_{\mathrm{FB}}$ for $\mathcal{O}\left(\alpha^{2}\right)$ and $\mathcal{O}\left(\alpha^{1}\right)$, is below the statistical error of $10^{-4}$. This can be treated as a measure of the missing QED photonic higher-order corrections in the KKMC predictions for $A_{\mathrm{FB}}$ for this particular type of experimental cutoff,

\footnotetext{
${ }^{30}$ Provided we trust the smallness of the technical precision error of KKMC.

${ }^{31}$ In particular, the non-IR parts of QED pentaboxes are missing; see Fig. 5 in Ref. [12].

${ }^{32}$ Differences in Fig. 18 are obtained using MC weights event per event, so statistical errors are grossly overestimated. This explains the lack of fluctuations among bins.
} 
KKMC: $A_{F B}^{|F|}\left(v_{\text {max }}\right)=A_{F B}^{|F| o n}\left(v_{\text {max }}\right)-A_{F B}^{\mid F l o f f}\left(v_{\text {max }}\right)$

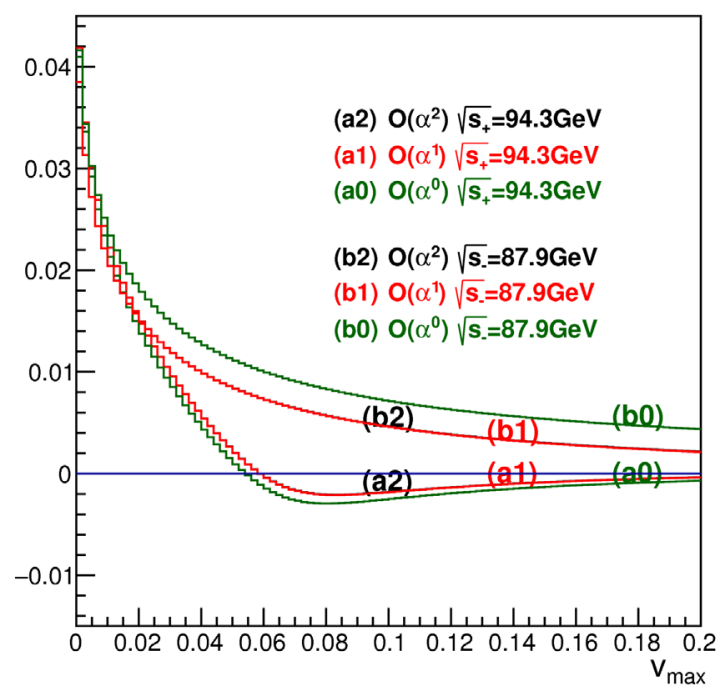

KKMC: $\quad \Delta A_{F B}^{\mathrm{FI}}\left(v_{\max }\right), \quad|\cos (\theta)|<0.90$

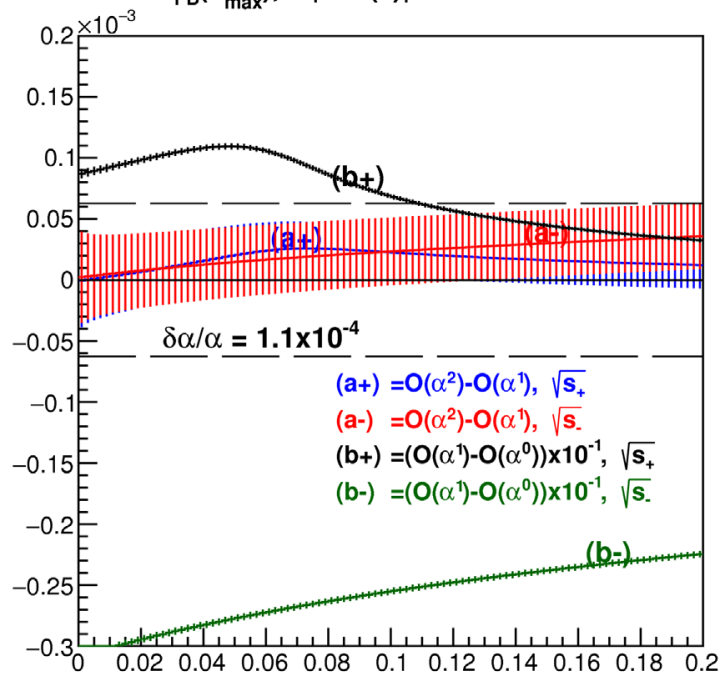

FIG. 18. IFI component in $A_{\mathrm{FB}}\left(s_{ \pm}\right)$obtained using KKMC program with three types of increasingly sophisticated QED matrix elements, $\mathcal{O}_{\exp }\left(\alpha^{i}\right), i=0,1,2$. The band between the dashed lines corresponds to the precision estimate of the $\alpha_{\mathrm{QED}}\left(M_{Z}\right)$ of Ref. [35].

$v_{\max }<0.2$ and $|\cos \theta|<0.9$, near $Z$ resonance, $\left|M_{Z}-\sqrt{s}\right| \leq 3.5 \mathrm{GeV}$.

Finally, let us remark that the $\mathrm{MC}$ results for $A_{\mathrm{FB}}$ presented here with a statistical precision of $10^{-4}$ were obtained using $\sim 10^{10} \mathrm{MC}$ events generated in parallel runs on PC farms. Reducing the statistical error to $10^{-5}$ will be feasible, but not trivial. However, higher precision may be also feasible with less $\mathrm{MC}$ events using the technique of recording differences of the $\mathrm{MC}$ weights, as it was done in some plots shown in the following.

KKMC CEEX, IFI off, $|\cos (\theta)|<1$

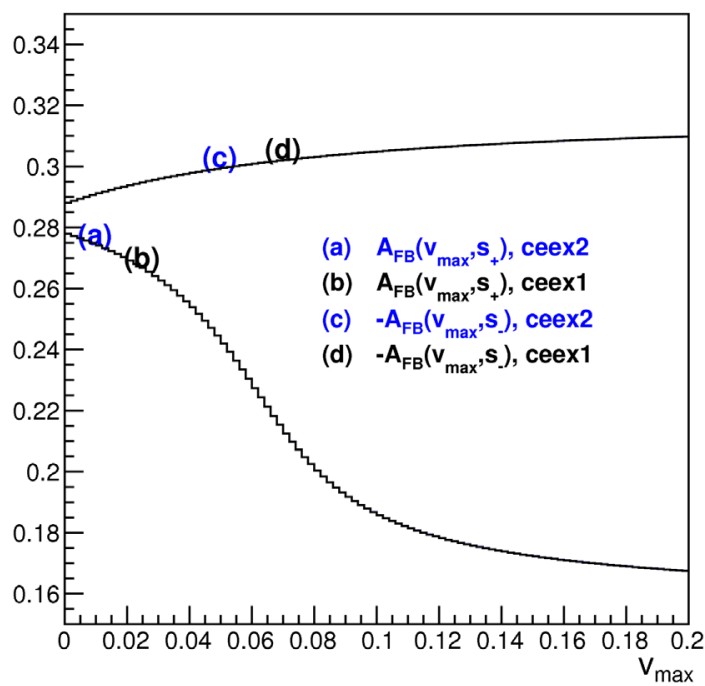

\section{G. More on the uncertainty of the ISR effect in $A_{\mathrm{FB}}$}

In this section, we will present a few results from KKMC which, in particular, will give us more insight on the ISR effects in $A_{\mathrm{FB}}$ when IFI is switched on and off.

In Fig. 19, we show differences between $\mathcal{O}_{\exp }\left(\alpha^{i}\right), i=$ 1,2 results from $\mathrm{KKMC}$ with the CEEX matrix element in the case of IFI switched off-that is pure ISR and FSR effects. In fact, the ISR effect is dominant here.

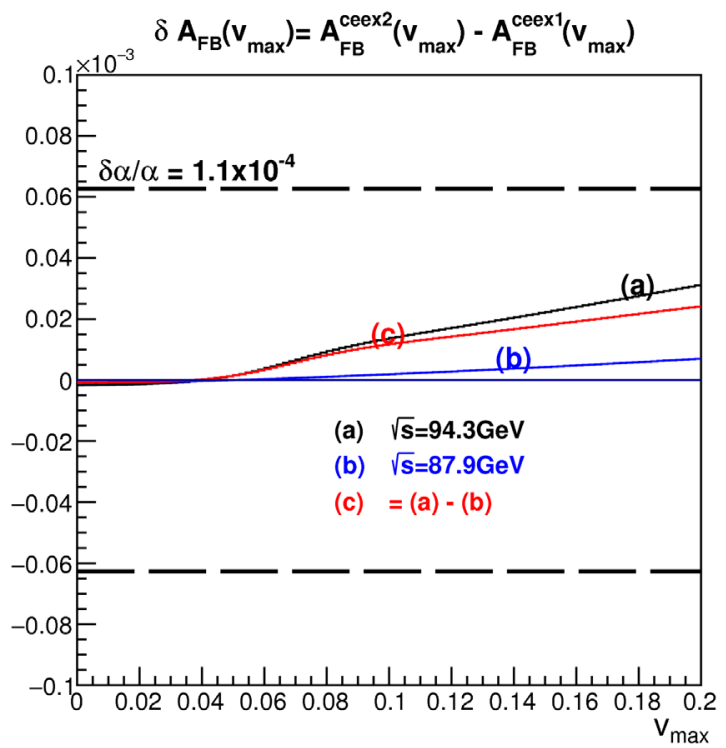

FIG. 19. Differences between $A_{\mathrm{FB}}$ calculated using CEEX matrix element $\mathcal{O}_{\exp }\left(\alpha^{i}\right), i=1,2$ with IFI switched off. The band between the dashed lines represents the precision estimate of the $\alpha_{\mathrm{QED}}\left(M_{Z}\right)$ of Ref. [35]. 
KKMC EEX without IFI, $|\cos (\theta)|<1$,

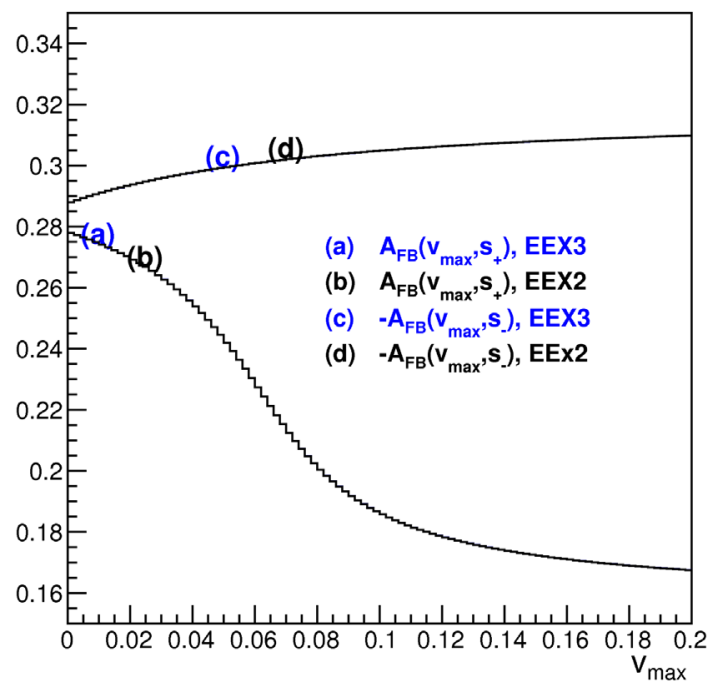

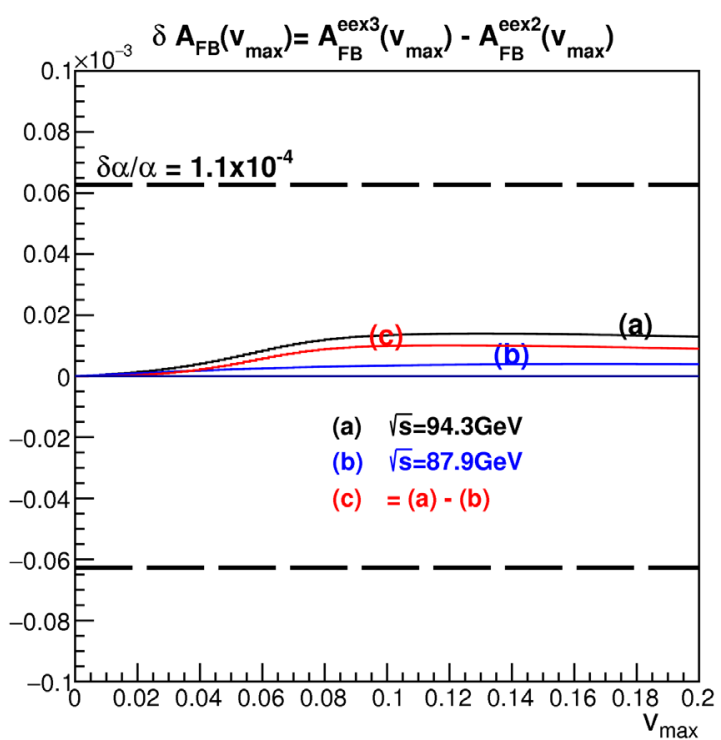

FIG. 20. Differences between $A_{\mathrm{FB}}$ calculated using the EEX matrix element $\mathcal{O}_{\exp }\left(\alpha^{i}\right), i=2,3$, which is without IFI.
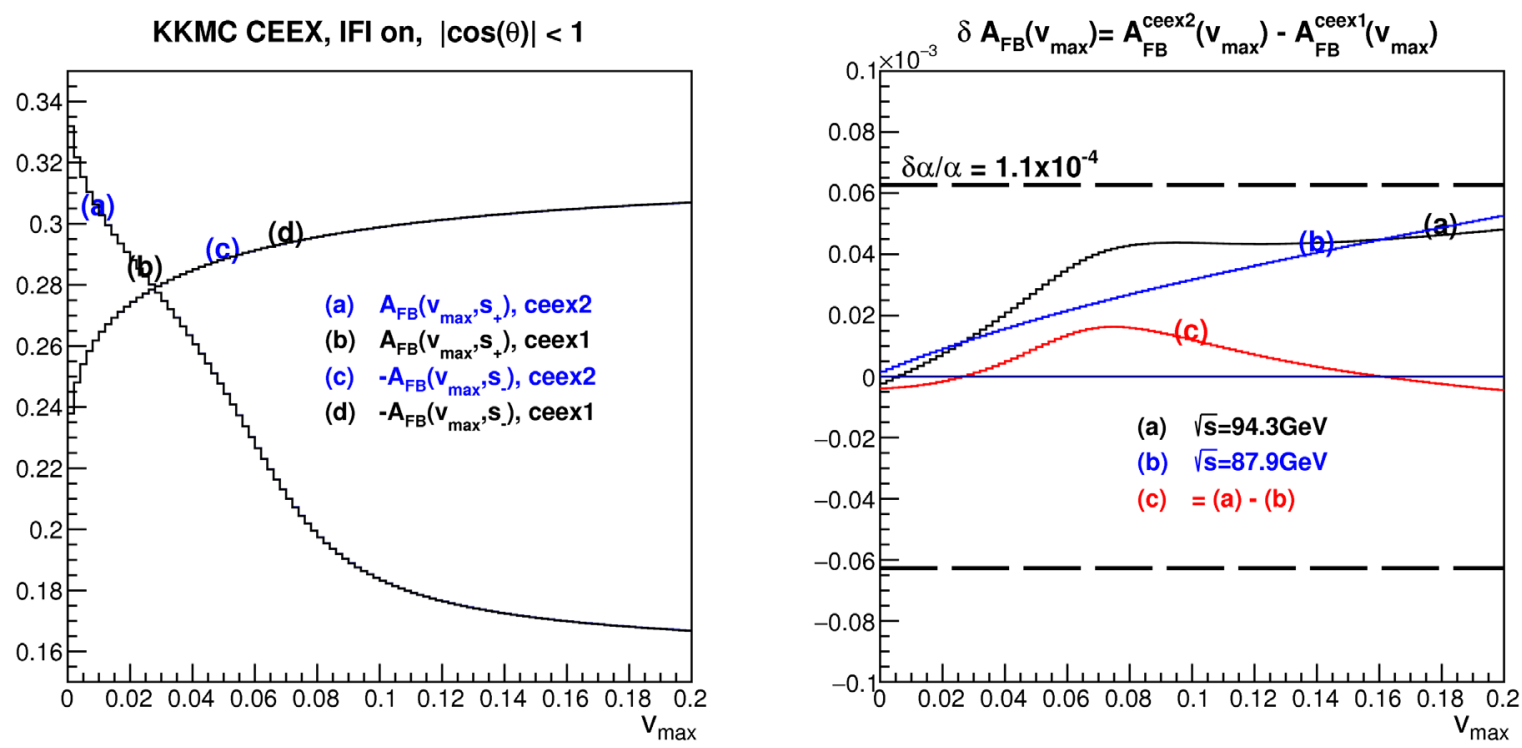

FIG. 21. Differences between $A_{\mathrm{FB}}$ calculated using the CEEX matrix element $\mathcal{O}_{\exp }\left(\alpha^{i}\right), i=1,2$ with IFI switched on.

The variation is smaller than $3 \times 10^{-5}$ and cancels between the two energies $\sqrt{s_{ \pm}}$. 33

The same phenomenon is seen in Fig. 20, albeit the differences are smaller, as expected. Note also that in both of the above cases, the effect of ISR is completely negligible for $v_{\max } \leq 0.05$, that is for cutoffs on photon energy interesting experimentally!

Finally, we switch on IFI and examine again the differences between $\mathcal{O}_{\exp }\left(\alpha^{i}\right), i=1,2$ results from KKMC with the CEEX matrix element in the case of IFI switched

\footnotetext{
${ }^{33}$ Such a cancellation of the ISR effect was already noticed in Ref. [5].
}

on. The results are shown in Fig. 21. This is the most interesting result, because it shows the indirect influence of ISR on the IFI contribution to $A_{\mathrm{FB}}$. Curve (c) shows that for the difference in $A_{\mathrm{FB}}$ between the two energies $\sqrt{s_{ \pm}}$, the first and second order results agree to within $\leq 2 \times 10^{-5}$. The disagreement is larger than was seen in the previous graph with IFI off in the semisoft region $v_{\max } \leq 0.06$.

One may conclude that the above result provides a strong indication that the QED uncertainty in $A_{\mathrm{FB}}$ from KKMC is of the order of the expected FCCee experimental error $\delta A_{\mathrm{FB}} \simeq 3 \times 10^{-5}$. In the above plots, statistical MC errors are negligible because all differences between the various QED matrix elements are calculated using weight differences for the same sets of weighted MC events. 
TABLE II. Table of the most important estimates of technical and physical uncertainties of $A_{\mathrm{FB}}$ due to IFI. We define $\Delta A_{\mathrm{FB}} \equiv A_{\mathrm{FB}}\left(s_{+}\right)-A_{\mathrm{FB}}\left(s_{-}\right)$. The quoted numbers represent maximum values for the $v_{\max } \in(0.0-0.05)$ and $v_{\max } \in(0.0-0.20)$ ranges in the corresponding figures.

\begin{tabular}{|c|c|c|c|c|c|c|}
\hline No. & Uncert. & IFI & Source & Ref. & $v_{\max } \leq 0.05$ & $v_{\max } \leq 0.20$ \\
\hline 1. & Tech. & OFF & $\left|A_{\mathrm{FB}}\left(s_{ \pm}\right)\right|_{\mathrm{KKMC}_{\text {ceex } 0}-\mathrm{KKsem} 0}$ & Fig. 11 & $2 \times 10^{-5}$ & $2 \times 10^{-5}$ \\
\hline 2. & Tech. & OFF & $\left|A_{\mathrm{FB}}\left(s_{ \pm}\right)\right|_{\mathrm{KKMC}_{\text {ceex } 2}-\mathrm{KKsem} 2}$ & Fig. 12 & $2 \times 10^{-5}$ & $3 \times 10^{-5}$ \\
\hline 3. & Phys. & $\mathrm{ON}$ & $\left|\Delta A_{\mathrm{FB}}^{\mathrm{IFI}}\right|_{\mathrm{KKMC}_{c c e 2}-\text { KKfoam5 }}$ & Fig. 17 & $2 \times 10^{-4}$ & $3 \times 10^{-4}$ \\
\hline 4. & Phys. & $\mathrm{ON}$ & $\left|A_{\mathrm{FB}}^{\mathrm{IFI}}\left(s_{+}\right)\right|_{\mathrm{CEEX} 2-\mathrm{CEEX} 1}$ & Fig. 18 & $0.3 \times 10^{-4}$ & $0.40 \times 10^{-4}$ \\
\hline 5. & Phys. & $\mathrm{ON}$ & $\left|A_{\mathrm{FB}}^{\mathrm{IFI}}\left(s_{-}\right)\right|_{\mathrm{CEEX} 2-\mathrm{CEEX} 1}$ & Fig. 18 & $0.3 \times 10^{-4}$ & $0.30 \times 10^{-4}$ \\
\hline 6. & Phys. & OFF & $\left|\Delta A_{\mathrm{FB}}^{\mathrm{CEEX} 2}-\Delta A_{\mathrm{FB}}^{\mathrm{CEEX} 1}\right|$ & Fig. 19 & $0.01 \times 10^{-5}$ & $0.20 \times 10^{-4}$ \\
\hline 7. & Phys. & $\mathrm{ON}$ & $\left|\Delta A_{\mathrm{FB}}^{\mathrm{CEEX} 2}-\Delta A_{\mathrm{FB}}^{\mathrm{CEEX} 1}\right|$ & Fig. 21 & $0.15 \times 10^{-4}$ & $0.15 \times 10^{-4}$ \\
\hline
\end{tabular}

\section{SUMMARY AND OUTLOOK}

The extensive numerical results presented in this work and summarized in Table II allow us to conclude that the technical and physical uncertainty of the prediction of KKMC for the IFI component of $A_{\mathrm{FB}}$ near the $Z$ resonance is of order $\sim 10^{-4}$ (row 3 in Table II). This is definitely better than the state of art in the LEP era $\sim 2 \times 10^{-3}-3 \times 10^{-3}$. For IFI switched off, the technical precision $\sim 10^{-5}$ was obtained (rows 1-2 in Table II). Some of the results presented here indicate that the physical precision (higher orders) of the IFI component of $A_{\mathrm{FB}}$ near the $Z$ resonance from KKMC is in fact at the level $\sim 1.5 \times 10^{-5}$ (row 7 in Table II). I.e., what is needed in the FCCee experiment proposed to measure the QED coupling constant at the scale $M_{Z}$ with this precision. This would allow $\alpha_{\mathrm{QED}}\left(M_{Z}\right)$ to be determined to a precision significantly better than the present estimate of Ref. [35], which is $\frac{\delta \alpha_{\mathrm{QED}}}{\alpha_{\mathrm{QED}}} \simeq 1.1 \times 10^{-4}$. However, more work is needed to achieve better confidence in the technical precision and higher order photonic QED corrections in the KKMC results. More work is also needed to estimate other missing nonphotonic QED corrections (e.g. pair emission) and electroweak corrections. Extension of the presented analysis to more realistic experimental selections (cuts) is also desirable.

The newly developed auxiliary MC program KKFOam was instrumental in the above achievement. For more precise tests of KKMC, it would be profitable to include in the phase space of KKFoam the exact contribution from nonsoft real $\mathcal{O}\left(\alpha^{1}\right)$ emission matched with semisoft analytic resummation.

\section{ACKNOWLEDGMENTS}

This work was supported in part by the Polish National Science Center Grant No. 2016/23/B/ST2/03927 and the CERN FCC Design Study Programme. S. A. Y. acknowledges support from The Citadel Foundation. The authors would like to thank Patrick Janot, Maciej Skrzypek and Zbigniew Was for critical reading of the manuscript and useful comments.

\section{APPENDIX A: FACTORIZING THE EXPONENTIATED FORMULA}

Starting from Eq. (12), let us introduce $\int d^{4} z \delta^{4}(z-y-$ $\left.x+x^{\prime}\right)=1, \quad \int d^{4} u \delta^{4}(u-y+x)=1$ and $\int d^{4} u^{\prime} \delta^{4}(u-$ $\left.y+x^{\prime}\right)=1$ in the Fourier expression, obtaining

$$
\begin{aligned}
\sigma(s)= & \frac{1}{\operatorname{flux}(s)} \sum_{V, V^{\prime}} \int \frac{d^{3} q_{1}}{q_{1}^{0}} \frac{d^{3} q_{2}}{q_{2}^{0}} \frac{d^{4} Q d^{4} x}{(2 \pi)^{4}} \frac{d^{4} Q^{\prime} d^{4} x^{\prime}}{(2 \pi)^{4}} e^{i x \cdot(P-Q)-i x^{\prime} \cdot\left(P-Q^{\prime}\right)} \frac{d^{4} y}{(2 \pi)^{4}} e^{i y \cdot\left(P-q_{1}-q_{2}\right)} \\
& \times \int \frac{d^{4} K d^{4} z}{(2 \pi)^{4}} \frac{d^{4} R d^{4} u}{(2 \pi)^{4}} \frac{d^{4} R^{\prime} d^{4} u^{\prime}}{(2 \pi)^{4}} e^{i\left(z-y-x+x^{\prime}\right) \cdot K+i(u-y-x) \cdot R+i\left(u^{\prime}-y+x^{\prime}\right) \cdot R^{\prime}} \\
& \times \exp \left\{\int \frac{d^{3} k}{k^{0}}\left[e^{-i k \cdot\left(y+x-x^{\prime}\right)} S_{I}(k)+e^{-i k \cdot(y+x)} S_{X}(k)+e^{-i k \cdot\left(y-x^{\prime}\right)} S_{X}(k)+e^{-i k \cdot y} S_{F}(k)\right]\right\} \\
& \times \exp \left\{\alpha B_{4}^{V}\left(Q^{2}, t, m_{\gamma}\right)+\alpha\left(B_{4}^{V^{\prime}}\left(Q^{\prime 2}, t, m_{\gamma}\right)\right)^{*}\right\} \mathcal{M}_{V}(Q, t) \mathcal{M}_{V^{\prime}}^{*}\left(Q^{\prime}, t\right) .
\end{aligned}
$$

The lowest-order spin amplitudes $\mathcal{M}_{V}, V=\gamma, Z$ are, up to a normalization constant, equal to the amplitudes $\mathfrak{M}_{\varepsilon \tau}^{V}$ defined in Appendix C, but fermion helicities are temporarily suppressed.

Thanks to the above reorganization, we may clearly factorize the result into contributions due to the ISR, FSR, and IFI components of multiphoton emission: 


$$
\begin{aligned}
& \sigma(s)=\frac{1}{\operatorname{flux}(s)} \int \frac{d^{3} q_{1}}{q_{1}^{0}} \frac{d^{3} q_{2}}{q_{2}^{0}} \frac{d^{4} Q d^{4} x}{(2 \pi)^{4}} \frac{d^{4} Q^{\prime} d^{4} x^{\prime}}{(2 \pi)^{4}} e^{i x \cdot(P-Q)-i x^{\prime} \cdot\left(P-Q^{\prime}\right)} \sum_{V, V^{\prime}} \\
& \times \int d^{4} K d^{4} R d^{4} R^{\prime} e^{i\left(-x+x^{\prime}\right) \cdot K+i(-x) \cdot R+i\left(+x^{\prime}\right) \cdot R^{\prime}} \\
& \times \int \frac{d^{4} z}{(2 \pi)^{4}} e^{i z \cdot K+\int \frac{d^{3} k}{k^{0}} e^{-i k \cdot z} S_{I}(k)} \int \frac{d^{4} u}{(2 \pi)^{4}} e^{i u \cdot R+\int \frac{d^{3} k}{k^{0}} e^{-i k \cdot u} S_{X}(k)} \\
& \times \int \frac{d^{4} u^{\prime}}{(2 \pi)^{4}} e^{i u^{\prime} \cdot R^{\prime}+\int \frac{d^{3} k}{k^{0}} e^{-i k \cdot u^{\prime}} S_{X}(k)} \int \frac{d^{4} y}{(2 \pi)^{4}} e^{i y \cdot\left(P-q_{1}-q_{2}-K-R-R^{\prime}\right)+\int \frac{d^{3} k}{k^{0}} e^{-i k \cdot y} S_{F}(k)} \\
& \times \exp \left\{\alpha B_{4}^{V}\left(Q^{2}, t, m_{\gamma}\right)+\alpha\left(B_{4}^{V^{\prime}}\left(Q^{\prime 2}, t, m_{\gamma}\right)\right)^{*}\right\} \mathcal{M}_{V}(Q) \mathcal{M}_{V^{\prime}}^{*}\left(Q^{\prime}\right) \\
& =\frac{1}{\operatorname{flux}(s)} \sum_{V, V^{\prime}} \int \frac{d^{3} q_{1}}{q_{1}^{0}} \frac{d^{3} q_{2}}{q_{2}^{0}} d^{4} Q d^{4} Q^{\prime} \\
& \times \int d^{4} K d^{4} R d^{4} R^{\prime} \delta^{4}(P-K-R-Q) \delta^{4}\left(P-K-R^{\prime}-Q^{\prime}\right) \\
& \times \int \frac{d^{4} z}{(2 \pi)^{4}} e^{i z \cdot K+\int \frac{d^{3} k}{k^{0}} e^{-i k \cdot z} S_{I}(k)} \int \frac{d^{4} u}{(2 \pi)^{4}} e^{i u \cdot R+\int \frac{d^{3} k}{k^{0}} e^{-i k \cdot u} S_{X}(k)} \\
& \times \int \frac{d^{4} u^{\prime}}{(2 \pi)^{4}} e^{i u^{\prime} \cdot R^{\prime}+\int \frac{d^{3} k}{k^{0}} e^{-i k \cdot u^{\prime}} S_{X}(k)} \int \frac{d^{4} y}{(2 \pi)^{4}} e^{i y \cdot\left(P-q_{1}-q_{2}-K-R-R^{\prime}\right)+\int \frac{d^{3} k}{k^{0}} e^{-i k \cdot y} S_{F}(k)} \\
& \times \exp \left\{\alpha B_{4}^{V}\left(Q^{2}, t, m_{\gamma}\right)+\alpha\left(B_{4}^{V^{\prime}}\left(Q^{\prime 2}, t, m_{\gamma}\right)\right)^{*}\right\} \mathcal{M}_{V}(Q) \mathcal{M}_{V^{\prime}}^{*}\left(Q^{\prime}\right) .
\end{aligned}
$$

\section{APPENDIX B: MAPPINGS IN THE FOAM INTEGRAND}

The regularized radiator distribution for the IFI component in the semisoft photon analytical exponentiation,

$$
\begin{aligned}
\rho(\gamma, v) & =F(\gamma)\left(\delta(v) \varepsilon^{\gamma}+\theta(v-\varepsilon) \gamma v^{\gamma-1}\right), \\
\int_{0}^{1} d v \rho(\gamma, v) & =F(\gamma) \equiv F_{\gamma},
\end{aligned}
$$

is valid for both positive and negative $\gamma$. The regulator $\varepsilon$ should be smaller that any scale dependence in the Born cross section times the target precision of the calculation. In our case it should be below $\Gamma_{Z} / M_{Z}$ by a factor of at least $10^{-4}$, i.e. $\varepsilon<10^{-5}$ is recommended. ${ }^{34}$ The distribution for FOAM should be positive in the exploration phase; hence,

$$
\tilde{\rho}(\gamma, v)=|\rho(\gamma, v)|=F_{\gamma}\left[\delta(v) \varepsilon^{\gamma}+\theta(v-\varepsilon)|\gamma| v^{\gamma-1}\right]
$$

is used. The mapping from $v$ to the internal variable $r \in$ $(0,1)$ of FOAM is chosen such that its Jacobian compensates exactly $\tilde{\rho}(v)$. More precisely, $v(r)$ is the solution of the equation

$$
r \int_{0}^{1} d v^{\prime} \tilde{\rho}\left(\gamma, v^{\prime}\right)=\int_{0}^{v} d v^{\prime} \tilde{\rho}\left(\gamma, v^{\prime}\right)=F_{\gamma} R(v) .
$$

\footnotetext{
${ }^{34}$ In the actual MC runs, we use $\varepsilon=10^{-6}$.
}

Note that for $\gamma>0$ we have $R(1)=1$, while for $\gamma<0$ we get $R(1)=2 e^{\gamma}-1>1$. Differentiating Eq. (B3) we get $F_{\gamma} R(1) d r=\tilde{\rho}(\gamma, v) d v$; hence the Jacobian is

$$
J(v)=|d v / d r|=F_{\gamma} R(1)(\tilde{\rho}(\gamma, v))^{-1} .
$$

For $\gamma>0$, the mapping [with $R(1)=1$ and $R(\varepsilon)=\varepsilon^{\gamma}$ ] is simply

$$
\begin{aligned}
& v(r)=0, \quad \text { for } r<R(\varepsilon)=\varepsilon^{\gamma}, \\
& v(r)=r^{1 / \gamma}, \quad \text { for } r>R(\varepsilon) .
\end{aligned}
$$

The corresponding Jacobian is

$$
\begin{aligned}
& J(v)=1 / R(\varepsilon)=\varepsilon^{-\gamma} \text { for } v=0 \text { and } \\
& J(v)=F_{\gamma}(\tilde{\rho}(\gamma, v))^{-1}=\frac{v}{r \gamma} \quad \text { for } v>\varepsilon .
\end{aligned}
$$

For $\gamma<0$ the mapping [with $R(1)=2 \varepsilon^{\gamma}-1$ and $\left.R(\varepsilon)=\varepsilon^{\gamma}\right]$ is more complicated:

$$
\begin{aligned}
v(r)= & 0, \quad \text { for } r<\frac{R(\varepsilon)}{R(1)}=\frac{\varepsilon^{\gamma}}{2 \varepsilon^{\gamma}-1}, \\
v(r)= & {[2 R(\varepsilon)-r R(1)]^{1 / \gamma}=\left[2 \varepsilon^{\gamma}-r\left(2 \varepsilon^{\gamma}-1\right)\right]^{1 / \gamma} } \\
& \text { for } r>\frac{R(\varepsilon)}{R(1)} .
\end{aligned}
$$


The corresponding Jacobian reads

$$
\begin{aligned}
& J(v)=\frac{R(1)}{R(\varepsilon)}=\frac{2 \varepsilon^{\gamma}-1}{\varepsilon^{\gamma}} \quad \text { for } v=0 \text { and } \\
& J(v)=\frac{F_{\gamma} R(1)}{\tilde{\rho}(\gamma, v)} \quad \text { for } v>\varepsilon .
\end{aligned}
$$

In the second simulation stage, FOAM generates weighted MC events with the IFI component being $w=J(v) \rho(\gamma, v)$. In the case of $\gamma>0$, the weight (component) in FOAM will be $w=1$ for any $v$, while for $\gamma<0$ it will be $w=R(1)$ for $v=0$ and $w=-R(1)$ for $v>\varepsilon$.

In addition, special care has to be taken in the case of $\gamma \rightarrow 0$, that is for $\cos \theta \simeq 0$, because in this region the above mappings can be numerically unstable due to the limited range of the exponent in floating-point arithmetic. Because of that, when $|\gamma \ln \varepsilon|<\Delta \ll 1$, many of the above formulas have to be expanded accordingly. ${ }^{35}$

For $|\gamma \ln \varepsilon|<\Delta \ll 1$ and $\gamma>0$, the expanded distribution, mapping, and Jacobian read:

$$
\begin{aligned}
& \tilde{\rho}(v)=\rho(v)=F_{\gamma}\left[\delta(v)(1+\gamma \ln \varepsilon)+\theta(v>\varepsilon) \frac{\gamma}{v}\right], \quad R(\varepsilon)=(1+\gamma \ln \varepsilon), \quad R(1)=1, \\
& v(r)=0 \quad \text { for } r<R(\varepsilon), \quad v(r)=\exp \left[-\frac{1}{\gamma}(1-r)\right] \quad \text { for } r>R(\varepsilon), \\
& J(v)=1 / R(\varepsilon) \quad \text { for } v=0 \quad \text { and } \quad J(v)=F_{\gamma}(\tilde{\rho}(\gamma, v))^{-1} \quad \text { for } v>\varepsilon .
\end{aligned}
$$

For $\gamma<0$, the expanded expressions with $R(\varepsilon)=1+\gamma \ln \varepsilon, R(1)=1+2 \gamma \ln \varepsilon>1$, read:

$$
\begin{aligned}
& \tilde{\rho}(v)=|\rho(v)|=F_{\gamma}\left[\delta(v)(1+\gamma \ln \varepsilon)-\theta(v>\varepsilon) \frac{\gamma}{v}\right], \\
& v(r)=0, \quad \text { for } r<\frac{R(\varepsilon)}{R(1)}=\frac{1+\gamma \ln \varepsilon}{1+2 \gamma \ln \varepsilon}, \\
& v(r)=\exp \left[\frac{1}{\gamma}(1-r) R(1)\right], \quad \text { for } r>\frac{R(\varepsilon)}{R(1)}, \\
& J(v)=\frac{R(1)}{R(\varepsilon)}=\text { for } v=0 \text { and } J(v)=\frac{F_{\gamma} R(1)}{\tilde{\rho}(\gamma, v)} \quad \text { for } v>\varepsilon .
\end{aligned}
$$

\section{APPENDIX C: ZERO AND FIRST ORDER AMPLITUDES WITHOUT RESUMMATION}

For constructing the semisoft photon analytical resummation and matching with the fixed-order $\mathcal{O}\left(\alpha^{1}\right)$ result, we need the zeroth and first order amplitudes and distributions in analytical form. In particular, we will need the differential cross section of the final muons, integrated over photon angles, but keeping control over the photon energy. The relevant results are scattered over several papers $[8,33,36]$. See also Refs. [7,9,37], where they are sometimes incomplete, or given in a form not suitable for our purposes; hence it is worth collecting them once more in this Appendix.

Following the notation of Ref. [33], the Born cross section and charge asymmetry read as follows:

$$
\begin{aligned}
\frac{d \sigma^{(0)}(s(1-v))}{d c} & =\frac{3 \sigma_{0}(s)}{8} \frac{1}{4} \sum_{\varepsilon, \tau= \pm}\left|\mathfrak{M}_{\varepsilon \tau}(v, c)\right|^{2}=\frac{3 \sigma_{0}(s)}{8}\left[\left(1+c^{2}\right) \mathfrak{D}(v)+2 c \overline{\mathfrak{D}}(v)\right], \\
\mathfrak{M}_{\varepsilon \tau}(v, c) & =\mathfrak{M}_{\varepsilon \tau}^{\gamma}(v, c)+\mathfrak{M}_{\varepsilon \tau}^{Z}(v, c)=(\varepsilon \tau+c) D_{\varepsilon, \tau}(v), \\
D_{\varepsilon, \tau}(v) & =D_{\varepsilon, \tau}^{\gamma}(v)+D_{\varepsilon, \tau}^{Z}(v)=\frac{q \tilde{q}}{1-v}+\frac{g_{\varepsilon} \tilde{g}_{\tau}}{\zeta-v}, \\
\zeta & =\frac{s-M_{Z}^{2}+i \Gamma_{Z} M_{Z}}{s}, \quad g_{\tau}=g_{V}+\tau g_{A}, \quad \tilde{g}_{\tau}=\tilde{g}_{V}+\tau \tilde{g}_{A}, \quad \sigma_{0}=\frac{4 \alpha \pi^{2}}{3 s},
\end{aligned}
$$

where $c=\cos \theta, q=Q_{e}, \tilde{q}=Q_{\mu}$ are electric charges, $\varepsilon, \tau= \pm$ are twice the helicity of $e^{-}$and $\mu^{-}$, and

\footnotetext{
${ }^{35}$ The value $\Delta=10^{-4}$ used now looks $\mathrm{OK}$, as the error of $\sim \Delta^{2}=10^{-8}$ is more than acceptable.
} 


$$
\begin{aligned}
\mathfrak{D}(v) & =\frac{1}{4} \sum_{\varepsilon \tau}\left|D_{\varepsilon, \tau}(v)\right|^{2}=\frac{c_{0}}{(1-v)^{2}}+\Re \frac{2 c_{1}}{(1-v)(\zeta-v)}+\frac{c_{2}}{|\zeta-v|^{2}}, \\
\mathfrak{D}(v) & =\frac{1}{4} \sum_{\varepsilon \tau} \varepsilon \tau\left|D_{\varepsilon, \tau}(v)\right|^{2}=\Re \frac{2 d_{1}}{(1-v)(\zeta-v)}+\frac{d_{2}}{|\zeta-v|^{2}}, \\
c_{0} & \left.=(q \tilde{q})^{2}, \quad c_{1}=q \tilde{q} g_{v} \tilde{g}_{v}, \quad c_{2}=\left(g_{v}^{2}+g_{a}^{2}\right)\left(\tilde{g}_{v}^{2}+\tilde{g}_{a}^{2}\right)\right), \\
d_{1} & =q \tilde{q} g_{a} \tilde{g}_{a}, \quad d_{2}=4 g_{v} g_{a} \tilde{g}_{v} \tilde{g}_{a} .
\end{aligned}
$$

The integration over $\cos \theta$ results in

$$
\begin{aligned}
& \sigma^{(0)}=\sigma_{0} \frac{1}{4} \sum_{\varepsilon \tau}\left|D_{\varepsilon, \tau}(0)\right|^{2}, \quad \sigma^{\Delta(0)}=\int 2 \cos \theta^{\Delta} d \sigma^{(1)}=\sigma_{0} \frac{1}{4} \sum_{\varepsilon \tau} \varepsilon \tau\left|D_{\varepsilon, \tau}(0)\right|^{2} \\
& A_{\mathrm{FB}}^{(0)}=\frac{3}{4}\left\langle 2 \cos \theta^{\Delta}\right\rangle^{(0)}=\frac{3}{4} \frac{\int 2 \cos \theta^{\Delta} d \sigma^{(0)}}{\sigma^{(0)}}=\frac{3}{4} \frac{\sum_{\varepsilon \tau} \varepsilon \tau\left|D_{\varepsilon, \tau}(0)\right|^{2}}{\sum_{\varepsilon \tau}\left|D_{\varepsilon, \tau}(0)\right|^{2}}=\frac{3}{4} \frac{\overline{\mathfrak{D}}(0)}{\mathfrak{D}(0)}
\end{aligned}
$$

Following the notation of Ref. [33], the noninterference $\mathcal{O}\left(\alpha^{1}\right)$ results with implicit integration over photon angles and explicit integration over photon energy up to $x=v_{\max }$ read:

$$
\begin{aligned}
\bar{A}_{\mathrm{FB}}^{(1)}(x) & =\frac{3}{4} \frac{\bar{\sigma}^{\Delta(1)}(x)}{\bar{\sigma}^{(1)}(x)}, \quad \bar{\sigma}^{\Delta(1)}(x)=\int_{v<x} 2 \cos \theta^{\Delta} d \bar{\sigma}^{(1)}, \\
\frac{\bar{\sigma}^{(1)}(x)}{\sigma_{0}} & =[1+F(x)] \mathfrak{D}(0)+W(x), \quad \frac{\bar{\sigma}^{\Delta(1)}(x)}{\sigma_{0}}=\left[1+F^{\Delta}(x)\right] \overline{\mathfrak{D}}(0)+W^{\Delta}(x), \\
W(x) & =\int_{0}^{x} d v\left[\gamma_{I}(s) P(v)+q^{2} \frac{\alpha}{\pi} \Delta_{s} \delta(v)\right](1-v) \mathfrak{D}(v), \\
W^{\Delta}(x) & =\int_{0}^{x} d v\left[\gamma_{I}(s) P(v)+q^{2} \frac{\alpha}{\pi} \Delta_{s} \delta(v)-q^{2} \frac{\alpha}{\pi} v\right](1-v) \overline{\mathfrak{D}}(v), \\
F(x) & =\int_{0}^{x} d v\left[\gamma_{F}(s(1-v)) P(v)+\tilde{q}^{2} \frac{\alpha}{\pi} \Delta_{s} \delta(v)\right], \\
F^{\Delta}(x) & =\int_{0}^{x} d v\left[\gamma_{F}(s(1-v)) P(v)+\tilde{q}^{2} \frac{\alpha}{\pi} \Delta_{s} \delta(v)-\tilde{q}^{2} \frac{\alpha}{\pi} v\right] \\
P(v) & =\left(\frac{1+(1-v)^{2}}{v}\right)_{+}=-\delta(v) \frac{3}{4} \ln \frac{1}{\varepsilon}+\theta(v-\varepsilon) \frac{1+(1-v)^{2}}{3}
\end{aligned}
$$

In Ref. [33], analytical integrations over $v$ were done, but for the purpose of the present resummation, we are more interested in the above unintegrated version.

In Ref. [8], the contribution of IFI was added to the above charge asymmetry, but in a version that was integrated over $v$. The unintegrated version ${ }^{36}$ including ISR + FSR + IFI with complete $\mathcal{O}\left(\alpha^{1}\right)$ for $v \in(0,1)$, needed for resummation is as follows:

\footnotetext{
${ }^{36}$ The unintegrated version of $U_{\varepsilon, \tau}^{\Delta}(x)$ was obviously used in Ref. [8], but was not explicitly shown there. Also, $U_{\varepsilon, \tau}(x)$ was not provided there.
} 


$$
\begin{aligned}
A_{\mathrm{FB}}^{(1)}(x) & =\frac{3}{4} \frac{\sigma^{\Delta(1)}(x)}{\sigma^{(1)}(x)}=\frac{3}{4} \frac{\int_{v<x} \cos \theta^{\Delta} d \sigma^{(1)}}{\sigma^{(1)}(x)}, \\
\sigma^{(1)}(x) & =\bar{\sigma}^{(1)}(x)+\sigma_{0} U(x), \quad \sigma^{\Delta(1)}(x)=\bar{\sigma}^{\Delta(1)}(x)+\sigma_{0} U^{\Delta}(x), \\
U(x) & =\int_{0}^{x} d v \rho_{X}^{(1)}(v)(1-v) \overline{\mathfrak{D}}(v, 0)+3 q \tilde{q} \frac{\alpha}{\pi} \Re\left\{A_{\gamma} \overline{\mathfrak{B}}^{\gamma}(0)+A_{Z} \overline{\mathfrak{B}}^{Z}(0)\right\}, \\
U^{\Delta}(x) & =\int_{0}^{x} d v \rho_{X}^{\Delta(1)}(v)(1-v) \mathfrak{D}(v, 0)+2 q \tilde{q} \frac{\alpha}{\pi} \Re\left\{A_{\gamma}^{\Delta} \mathfrak{B}^{\gamma}(0)+A_{Z}^{\Delta} \mathfrak{B}^{Z}(0)\right\}, \\
\rho_{X}^{(1)}(v) & =2 q \tilde{q} \frac{\alpha}{\pi}\left\{\delta(v)\left[3 \ln \frac{1}{\delta}\right]+\theta(v-\delta)(-3) \frac{2-v}{2 v}\right\} \\
\rho_{X}^{\Delta(1)}(v) & =2 q \tilde{q} \frac{\alpha}{\pi}\left\{\delta(v)\left[5 \ln \frac{1}{\delta}\right]+\theta(v-\delta) \frac{(-1)}{(2-v) v}\left[10(1-v)+3 v^{2}\right]\right\} .
\end{aligned}
$$

The combined contributions to the total cross section from real soft emission (interference part) and virtual $\gamma \gamma$ and $\gamma Z$ boxes can be deduced from the $k_{\max } \rightarrow 0$ limit of formulas in Ref. [36]:

$$
\begin{aligned}
A_{\gamma} & =-\frac{1}{2}, \quad A_{Z}=-\ln |1-\zeta|-\zeta+(1-\zeta)(2-\zeta) \ln \frac{-\zeta}{1-\zeta} . \\
\mathfrak{B}^{\gamma}(0) & =c_{0}+\frac{c_{1}}{\zeta^{*}}, \quad \mathfrak{B}^{Z}(0)=\frac{c_{1}}{\zeta^{*}}+\frac{c_{2}}{\zeta \zeta^{*}} .
\end{aligned}
$$

The analogous contributions to $\sigma^{\triangle(1)}$ can be obtained from formulas in Ref. [8]:

$$
\begin{aligned}
A_{\gamma}^{\Delta}= & \frac{65}{36}-i \frac{2}{3} \pi, \\
A_{Z}^{\Delta}= & \frac{31}{9} \zeta-9 \zeta^{2}+4 \zeta^{3}-\ln (1-\zeta)\left(\frac{15}{2}-13 \zeta+12 \zeta^{2}-4 \zeta^{3}\right) \\
& +\ln (-\zeta)\left(5-\frac{17}{3} \zeta+2 \zeta^{2}\right)+4 \zeta(1-\zeta)^{3}\left(\operatorname{Li}_{2}\left(\frac{-\zeta}{1-\zeta}\right)-\frac{\pi^{2}}{6}\right) . \\
\overline{\mathfrak{B}}^{\gamma}(0)= & \frac{d_{1}}{\zeta^{*}}, \quad \overline{\mathfrak{B}}^{Z}(0)=\frac{d_{1}}{\zeta^{*}}+\frac{d_{2}}{\zeta \zeta^{*}} .
\end{aligned}
$$

The following combinations of the Born amplitudes are involved:

$$
\begin{aligned}
\mathfrak{D}(v, u) & =\Re \frac{1}{4} \sum_{\varepsilon \tau}\left(D_{\varepsilon, \tau}(v)^{*} D_{\varepsilon, \tau}(u)\right) \\
& =\Re\left\{\frac{c_{0}}{(1-v)(1-u)}+\frac{c_{1}}{(1-v)\left(\zeta^{*}-u\right)}+\frac{c_{1}}{(\zeta-v)(1-u)}+\frac{c_{2}}{(\zeta-v)\left(\zeta^{*}-u\right)}\right\} \\
\overline{\mathfrak{D}}(v, u) & =\Re \frac{1}{4} \sum_{\varepsilon \tau} \varepsilon \tau\left(D_{\varepsilon, \tau}(v)^{*} D_{\varepsilon, \tau}(u)\right) \\
& =\Re\left\{\frac{d_{1}}{(1-v)\left(\zeta^{*}-u\right)}+\frac{d_{1}}{(\zeta-v)(1-u)}+\frac{d_{2}}{(\zeta-v)\left(\zeta^{*}-u\right)}\right\}, \\
\mathfrak{B}^{V}(0) & =\frac{1}{4} \sum_{\varepsilon \tau} D_{\varepsilon, \tau}^{V}(0)^{*} D_{\varepsilon, \tau}(0), \quad \overline{\mathfrak{B}}^{V}(0)=\frac{1}{4} \sum_{\varepsilon \tau} \varepsilon \tau D_{\varepsilon, \tau}^{V}(0)^{*} D_{\varepsilon, \tau}(0), \quad V=\gamma, Z .
\end{aligned}
$$

Let us remark that the following relations hold:

$$
\begin{aligned}
\mathfrak{D}(v)=\mathfrak{D}(v, v), & \overline{\mathfrak{D}}(v)=\overline{\mathfrak{D}}(v, v), \\
\mathfrak{R} \mathfrak{B}^{\gamma}(0)+\mathfrak{R} \mathfrak{B}^{Z}(0)=\mathfrak{D}(0), & \mathfrak{R} \overline{\mathfrak{B}}^{\gamma}(0)+\mathfrak{R} \overline{\mathcal{B}}^{Z}(0)=\overline{\mathfrak{D}}(0) .
\end{aligned}
$$


We also need the virtual box and real soft contributions before integration over $c=\cos \theta$. Spin amplitudes for two $\gamma \gamma$ box diagram and two $\gamma Z$ box diagram contributions, normalized the same way as the Born spin amplitudes, read as follows:

$$
\begin{aligned}
& \mathfrak{M}_{\varepsilon \tau}^{\{\gamma \gamma\}}=(q \tilde{q})^{2}\left(\varepsilon \tau X_{1}(c)+X_{2}(c)\right), \\
& \mathfrak{M}_{\varepsilon \tau}^{\{\gamma Z\}}=q \tilde{q} g_{\varepsilon} q_{\tau}\left(\varepsilon \tau Z_{1}(c)+Z_{2}(c)\right) .
\end{aligned}
$$

Their interference with Born amplitudes leads to the following contributions: ${ }^{37}$

$$
\begin{aligned}
\frac{d \sigma^{\gamma \gamma}}{d c} & =\frac{3 \sigma_{0}}{8} \frac{1}{4} \sum_{\varepsilon \tau} 2 \Re\left[\mathfrak{M}_{\varepsilon \tau}^{\{\gamma \gamma\}} \mathfrak{M}_{\varepsilon \tau}^{*}(0, c)\right] \\
& =\frac{3 \sigma_{0}}{8} \frac{1}{4} \sum_{\varepsilon \tau} 2 \Re\left\{(q \tilde{q})^{2}\left[X_{1}(c)+c X_{2}(c)+\varepsilon \tau\left(c X_{1}+X_{2}(c)\right)\right] D_{\varepsilon \tau}^{*}(0)\right\} \\
& =\frac{3 \sigma_{0}}{8} q \tilde{q} 2 \Re\left\{\left(c_{0}+\frac{c_{1}}{\zeta^{*}}\right)\left[X_{1}(c)+c X_{2}(c)\right]+\frac{d_{1}}{\zeta^{*}}\left[c X_{1}(c)+X_{2}(c)\right]\right\} \\
& =\frac{3 \sigma_{0}}{8} q \tilde{q} 2 \Re\left\{\left(c_{0}+\frac{c_{1}}{\zeta^{*}}\right)\left[F^{\gamma \gamma}(c)-F^{\gamma \gamma}(-c)\right]+\frac{d_{1}}{\zeta^{*}}\left[F^{\gamma \gamma}(c)+F^{\gamma \gamma}(-c)\right]\right\},
\end{aligned}
$$

where

$$
\begin{aligned}
X_{1}(c)+c X_{2}(c) & =F^{\gamma \gamma}(c)-F^{\gamma \gamma}(-c), \\
c X_{1}(c)+X_{2}(c) & =F^{\gamma \gamma}(c)+F^{\gamma \gamma}(-c), \quad c_{ \pm}=\frac{1 \pm c}{2}, \\
F^{\gamma \gamma}(c) & =2 \frac{\alpha}{\pi}\left\{2\left(\ln \frac{m_{\gamma}^{2}}{s}+i \pi\right) c_{+}^{2} \ln \frac{c_{-}}{c_{+}}-\frac{1}{2} c\left(\ln ^{2} c_{-}+2 i \pi\right)+c_{+}\left(\ln c_{-}+i \pi\right)\right\} .
\end{aligned}
$$

Similarly, for the $\gamma Z$ box, we have:

$$
\begin{aligned}
\frac{d \sigma^{\gamma Z}}{d c} & =\frac{3 \sigma_{0}}{8} \frac{1}{4} \sum_{\varepsilon \tau} 2 \Re\left[\mathfrak{M}_{\varepsilon \tau}^{\{\gamma Z\}} \mathfrak{M}_{\varepsilon \tau}^{*}(0, c)\right] \\
& =\frac{3 \sigma_{0}}{8} \frac{1}{4} \sum_{\varepsilon \tau} 2 \Re\left\{q \tilde{q}\left[Z_{1}(c)+c Z_{2}(c)+\varepsilon \tau\left(c Z_{1}+Z_{2}(c)\right)\right] D_{\varepsilon \tau}^{*}(0)\right\} \\
& =\frac{3 \sigma_{0}}{8} q \tilde{q} 2 \Re\left\{\left(c_{1}+\frac{c_{2}}{\zeta^{*}}\right)\left[Z_{1}(c)+c Z_{2}(c)\right]+\left(d_{1}+\frac{d_{2}}{\zeta^{*}}\right)\left[c Z_{1}(c)+Z_{2}(c)\right]\right\} \\
& =\frac{3 \sigma_{0}}{8} q \tilde{q} 2 \Re\left\{\left(c_{1}+\frac{c_{2}}{\zeta^{*}}\right)\left[F^{\gamma Z}(c)-F^{\gamma Z}(-c)\right]+\left(d_{1}+\frac{d_{2}}{\zeta^{*}}\right)\left[F^{\gamma Z}(c)+F^{\gamma Z}(-c)\right]\right\},
\end{aligned}
$$

where $F^{\gamma Z}(c)$ is related in a simple way to $f(s, t, u)$ of Ref. [38]:

$$
F^{\gamma Z}(c)=2 \frac{\alpha}{\pi} c_{+}^{2} s f(s, t, u), \quad F^{\gamma Z}(-c)=2 \frac{\alpha}{\pi} c_{-}^{2} s f(s, u, t) .
$$

In the KKMC code, the $\gamma Z$ box of Ref. [38] is programmed as follows:

$$
\begin{aligned}
F^{\gamma Z}(c)= & \ln \frac{t}{u} \ln \frac{m_{\gamma}^{2}}{(t u)^{1 / 2}}-2 \ln \frac{t}{u} \ln \frac{\bar{M}^{2}-s}{\bar{M}^{2}}+\operatorname{Li}_{2}\left(\frac{\bar{M}^{2}+u}{\bar{M}^{2}}\right)-\mathrm{Li}_{2}\left(\frac{\bar{M}^{2}+t}{\bar{M}^{2}}\right) \\
& +\frac{\left(\bar{M}^{2}-s\right)\left(u-t-\bar{M}^{2}\right)}{u^{2}}\left(\ln \frac{-t}{s} \ln \frac{\bar{M}^{2}-s}{\bar{M}^{2}}+\operatorname{Li}_{2}\left(\frac{\bar{M}^{2}+t}{\bar{M}^{2}}\right)-\mathrm{Li}_{2}\left(\frac{\bar{M}^{2}-s}{\bar{M}^{2}}\right)\right) \\
& +\frac{\left(\bar{M}^{2}-s\right)^{2}}{u s} \ln \frac{\bar{M}^{2}-s}{\bar{M}^{2}}+\frac{\left(\bar{M}^{2}-s\right)}{u} \ln \frac{-t}{\bar{M}^{2}},
\end{aligned}
$$

where $\bar{M}^{2}=M_{Z}^{2}-M_{Z} \Gamma_{Z}, t=-(1-c) s$ and $u=(1+c) s$.

\footnotetext{
${ }^{37}$ We use $(1 / 4) \sum_{\varepsilon \tau} \varepsilon \tau q \tilde{q} g_{\varepsilon} \tilde{g}_{\tau}=d_{1}=q \tilde{q} g_{a} \tilde{g}_{a}$ and $(1 / 4) \sum_{\varepsilon \tau} g_{\varepsilon} \tilde{g}_{\tau}=c_{1}=q \tilde{q} g_{v} \tilde{g}_{v}$.
} 

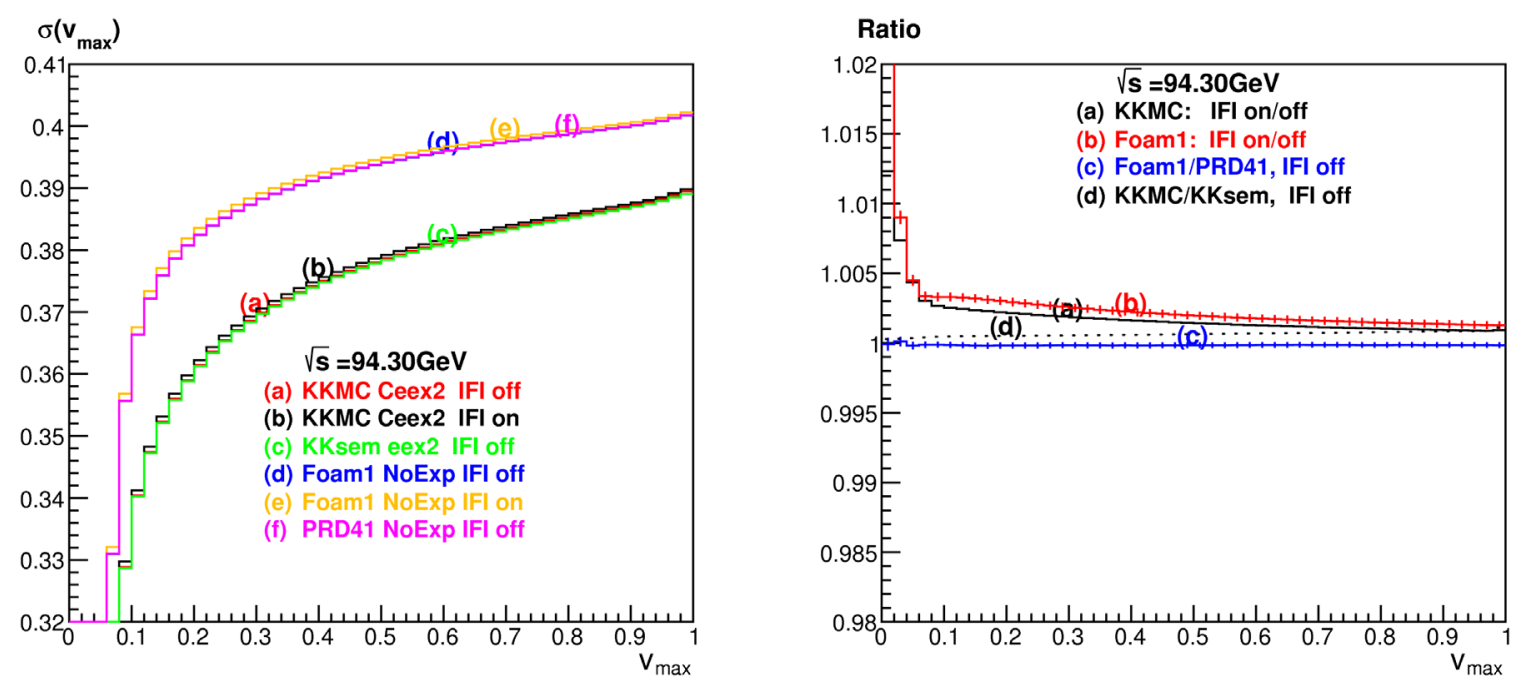

FIG. 22. $\mathcal{O}\left(\alpha^{1}\right)$ from old papers and KKFoam.

Finally, the above box contributions have to be combined with (interference) the corresponding soft real emission contribution:

$$
\begin{aligned}
\frac{d \sigma_{X}^{\mathrm{soft}}}{d c}= & \frac{d \sigma^{(0)}}{d c} q \tilde{q} \frac{\alpha}{\pi} \delta_{X}^{\mathrm{soft}}(c), \\
\delta_{X}^{\mathrm{soft}}(c)= & 4 \ln \frac{c_{-}}{c_{+}} \ln \frac{s^{1 / 2} \epsilon}{m_{\gamma}}+\ln ^{2} c_{-}-\ln ^{2} c_{+} \\
& +2 \mathrm{Li}_{2}\left(c_{+}\right)-2 \mathrm{Li}_{2}\left(c_{-}\right),
\end{aligned}
$$

such that the usual cancellation of the IR regulator $m_{\gamma}$ occurs, leaving out the IR cutoff on photon energy $v \leq \epsilon \ll 1$.

Let us finally define explicit relations between integrated and unintegrated virtual + soft contributions:

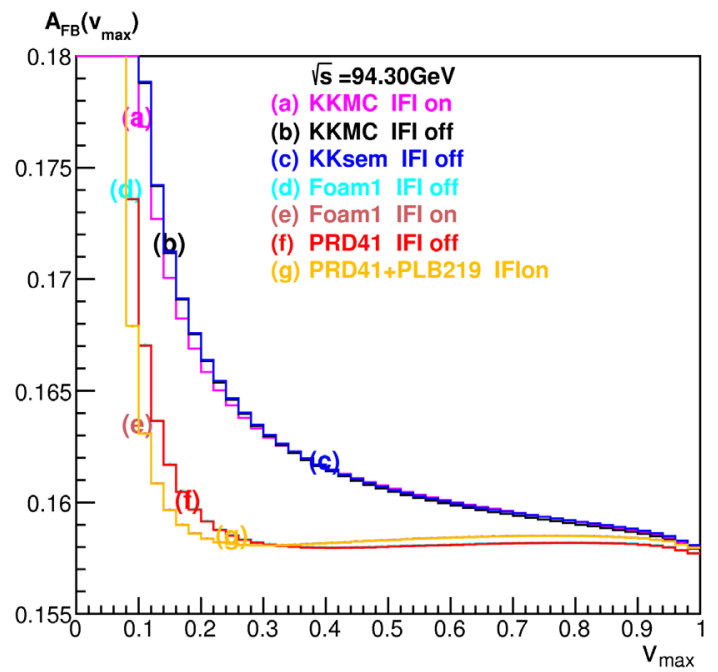

$$
\begin{aligned}
3 A_{\gamma} & =\int d c\left(F^{\gamma \gamma}(c)-F^{\gamma \gamma}(-c)+\delta_{X}^{\mathrm{soft}}(c)\right), \\
2 A_{\gamma}^{\Delta} & =\int 2 c d c\left(F^{\gamma \gamma}(c)+F^{\gamma \gamma}(-c)+\delta_{X}^{\mathrm{soft}}(c)\right), \\
3 A_{Z} & =\int d c\left(F^{\gamma Z}(c)-F^{\gamma \gamma}(-c)+\delta_{X}^{\mathrm{soft}}(c)\right), \\
2 A_{Z}^{\Delta} & =\int 2 c d c\left(F^{\gamma Z}(c)+F^{\gamma \gamma}(-c)+\delta_{X}^{\mathrm{soft}}(c)\right) .
\end{aligned}
$$

Finally, in Figs. 22 and 23 we crosscheck the old analytical results with KKFOam, in which the integration over $\cos \theta$ (virtual) and over photon energy $v$ (real photon) is done numerically. As we see, there is perfect agreement between old analytical formulas and new results using KKFOam.

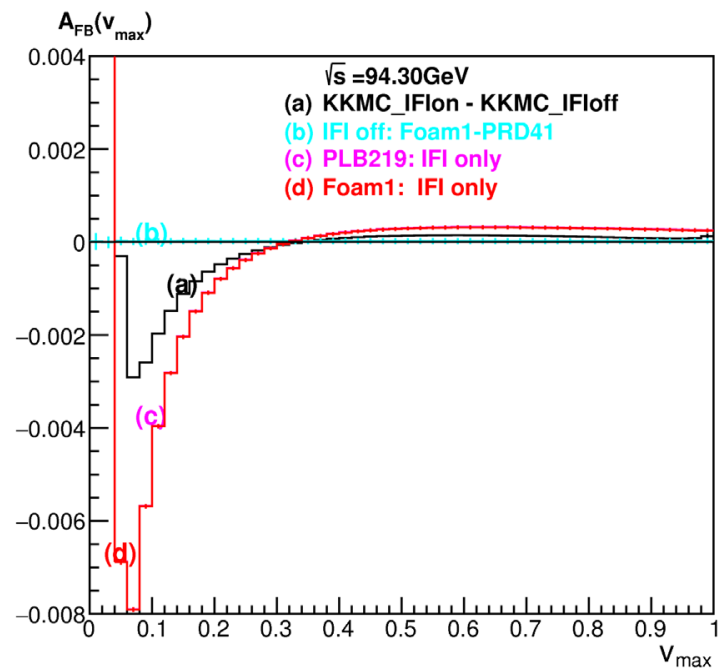

FIG. 23. $\mathcal{O}\left(\alpha^{1}\right)$ from old papers and KKFoam. 
[1] M. Bicer et al. (TLEP Design Study Working Group), First look at the physics case of TLEP, J. High Energy Phys. 01 (2014) 164.

[2] A. Abada et al. (FCC Collaboration), FCC physics opportunities, Eur. Phys. J. C 79, 474 (2019).

[3] A. Abada et al. (FCC Collaboration), FCC-ee: The lepton collider, Eur. Phys. J. ST 228, 261 (2019).

[4] A. Blondel et al., Theory requirements and possibilities for the FCC-ee and other future high energy and precision frontier lepton colliders, arXiv:1901.02648.

[5] P. Janot, Direct measurement of $\alpha_{\mathrm{QED}}\left(m_{Z}^{2}\right)$ at the FCC-ee, J. High Energy Phys. 02 (2016) 053.

[6] S. Schael et al. (ALEPH, DELPHI, L3, OPAL, SLD, LEP Electroweak Working Group, SLD Electroweak Group, SLD Heavy Flavour Group), Precision electroweak measurements on the $Z$ resonance, Phys. Rep. 427, 257 (2006).

[7] M. Böhm and W. Hollik, Forward-backward asymmetries, in Workshop on Z Physics at LEP1: General Meetings, Vol. 1: Standard Physics, edited by G. Altarelli et al. (CERN, Geneva, Switzerland, 1989), pp. 203-234, https:// cds.cern.ch/record/116932.

[8] S. Jadach and Z. Wąs, Suppression of QED interference contributions to the charge asymmetry at the $Z^{0}$ resonance, Phys. Lett. B 219, 103 (1989).

[9] D. Yu. Bardin, M. Bilenky, A. Chizhov, A. Sazonov, O. Fedorenko, T. Riemann, and M. Sachwitz, Analytic approach to the complete set of QED corrections to fermion pair production in $e^{+} e^{-}$annihilation, Nucl. Phys. B351, 1 (1991).

[10] D. Yu. Bardin, M. Grunewald, and G. Passarino, Precision calculation project report, arXiv:hep-ph/9902452.

[11] M. Kobel et al. (Two Fermion Working Group), TwoFermion production in electron-positron collisions, in Proceedings, Monte Carlo Workshop: Report of the Working Groups on Precision Calculation for LEP-2 Physics, edited by G. Passarino et al. (CERN, Geneva, Switzerland, 2000), pp. 269-378, http://cds.cern.ch/record/456118.

[12] S. Jadach, B. F. L. Ward, and Z. Was, Coherent exclusive exponentiation for precision Monte Carlo calculations, Phys. Rev. D 63, 113009 (2001).

[13] D. Yu. Bardin, M. Bilenky, P. Christova, M. Jack, L. Kalinovskaya, A. Olchevski, S. Riemann, and T. Riemann, ZFITTER v.6.21: A semi-analytical program for fermion pair production in $e^{+} e^{-}$annihilation, Comput. Phys. Commun. 133, 229 (2001).

[14] G. Montagna, O. Nicrosini, G. Passarino, and F. Piccinini, TOPAZO 2.0 - A program for computing de-convoluted and realistic observables around the $Z^{0}$ peak, Comput. Phys. Commun. 93, 120 (1996).

[15] G. Montagna, F. Piccinini, O. Nicrosini, G. Passarino, and R. Pittau, On a semi-analytical and realistic approach to $e^{+} e^{-}$annihilation into fermion pairs and to Bhabha scattering within the minimal standard model at LEP energies, Nucl. Phys. B401, 3 (1993).

[16] S. Jadach, B. F. L. Ward, and Z. Was, The Monte Carlo program KORALZ version 4.0 for lepton or quark pair production at LEP/SLC energies, Comput. Phys. Commun. 79, 503 (1994).
[17] S. Jadach, B. F. L. Ward, and Z. Was, The Monte Carlo program KORALZ, for the lepton or quark pair production at LEP/SLC energies From version 4.0 to version 4.04, Comput. Phys. Commun. 124, 233 (2000).

[18] M. Greco, G. Pancheri-Srivastava, and Y. Srivastava, Radiative corrections for colliding beam resonances, Nucl. Phys. B101, 234 (1975).

[19] M. Greco, G. Pancheri-Srivastava, and Y. Srivastava, Radiative effects for resonances with applications to colliding beam processes, Phys. Lett. 56B, 367 (1975).

[20] M. Greco, G. Pancheri-Srivastava, and Y. Srivastava, Radiative corrections to $e^{+} e^{-} \rightarrow \mu^{+} \mu^{-}$around the $Z^{0}$, Nucl. Phys. B171, 118 (1980); Erratum B197, 543 (1982).

[21] S. Jadach, B. F. L. Ward, and Z. Was, Coherent exclusive exponentiation CEEX: the case of the resonant $e^{+} e^{-}$ collision, Phys. Lett. B 449, 97 (1999).

[22] S. Jadach, B.F. L. Ward, and Z. Was, The precision Monte Carlo event generator $\mathrm{KK}$ for two-fermion final states in $e^{+} e^{-}$collisions, Comput. Phys. Commun. 130, 260 (2000).

[23] S. Jadach, M. Skrzypek, and B. Pietrzyk, On the precision of calculations of initial state radiation in the LEP $Z$ line-shape fits, Phys. Lett. B 456, 77 (1999).

[24] A. Blondel et al., Standard Model theory for the FCC-ee: The Tera-Z, arXiv:1809.01830.

[25] D. R. Yennie, S. C. Frautschi, and H. Suura, The infrared divergence phenomena and high-energy processes, Ann. Phys. (N.Y.) 13, 379 (1961).

[26] S. Jadach, W. Paczek, M. Skrzypek, B. F. L. Ward, and S. A. Yost, The path to $0.01 \%$ theoretical luminosity precision for the FCC-ee, Phys. Lett. B 790, 314 (2019).

[27] S. Jadach and M. Skrzypek, QED challenges at FCC-ee precision measurements, arXiv:1903.09895.

[28] D. Y. Bardin, M. S. Bilenky, T. Riemann, M. Sachwitz, H. Vogt, and P. Ch. Christova, DIZET-Electroweak one-loop corrections for $e^{+}+e^{-} \rightarrow f^{+}+f^{-}$around the $Z^{0}$ peak, Comput. Phys. Commun. 59, 303 (1990).

[29] S. Jadach, Foam: Multi-dimensional general purpose Monte Carlo generator with self-adapting simplical grid, Comput. Phys. Commun. 130, 244 (2000).

[30] S. Jadach, Foam: A general-purpose cellular Monte Carlo event generator, Comput. Phys. Commun. 152, 55 (2003).

[31] S. Jadach, B. F. L. Ward, and Z. Was, The Monte Carlo program KORALZ, version 3.8, for the lepton or quark pair production at LEP/SLC energies, Comput. Phys. Commun. 66, 276 (1991).

[32] Z-Physics at LEP1, edited by G. Altarelli, R. Kleiss, and C. Verzegnassi (CERN, Geneva, 1989), Vol. 1-3.

[33] Z. Wąs and S. Jadach, First- and higher-order noninterference QED radiative corrections to the charge asymmetry at the $Z$ resonance, Phys. Rev. D 41, 1425 (1990).

[34] R. Kleiss and W. J. Stirling, Spinor techniques for calculating $p p \rightarrow W^{ \pm} / Z^{0}+$ jets, Nucl. Phys. B262, 235 (1985).

[35] M. Davier, A. Hoecker, B. Malaescu, and Z. Zhang, Reevaluation of the hadronic contributions to the muon 
$g-2$ and to $\alpha\left(M_{Z}^{2}\right)$, Eur. Phys. J. C 71, 1515 (2011); Erratum 72, 1874 (2012).

[36] S. Jadach, J. H. Kuhn, R. G. Stuart, and Z. Wąs, QCD and QED corrections to the longitudinal polarization asymmetry, Z. Phys. C 38, 609 (1988); Erratum 45, 528 (1990).
[37] D. Yu. Bardin et al., QED corrections with partial angular integration to fermion pair production in $e^{+} e^{-}$annihilation, Phys. Lett. B 255, 290 (1991).

[38] R. W. Brown, R. Decker, and E. A. Paschos, Weak Corrections to the $e^{+} e^{-} \rightarrow \mu^{+} \mu^{-}$Asymmetry, Phys. Rev. Lett. 52, 1192 (1984). 